Supporting Information

\title{
Bimetallic Photoredox Catalysis: Visible Light-Promoted Aerobic Hydroxylation of Arylboronic Acids with a Dirhodium(II) Catalyst
}

Hsiang-Ming Wang, ${ }^{\dagger}$ Ming-Lun Liu, ${ }^{\dagger}$ Jing-Wen Tu, ${ }^{\dagger}$ Emily MiuraStempel, ${ }^{\ddagger}$ Michael G. Campbell, ${ }^{*,}$ and Gary Jing Chuang ${ }^{*, \dagger}$

${ }^{\dagger}$ Department of Chemistry, Chung Yuan Christian University, Chung-Li, Taiwan

${ }^{\ddagger}$ Department of Chemistry, Barnard College, New York, New York 10027, United States

*mcampbel@barnard.edu

*gjchuang@cycu.edu.tw 


\section{Table of Contents}

Visible Light Photoreaction Setup..................................................................... 4

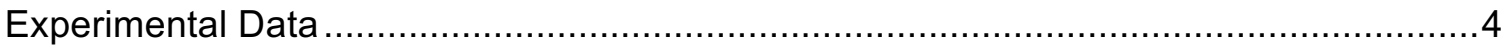

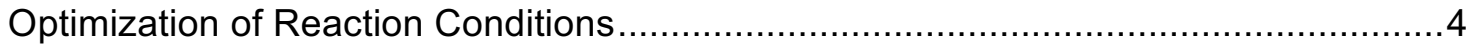

Table S1. Background reaction comparison .................................................. 4

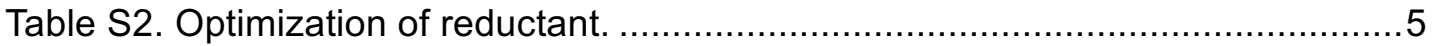

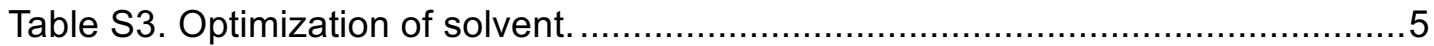

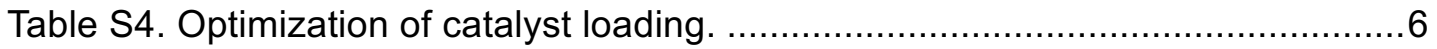

Light Source On-Off Experiment (data pertaining to Figure 1) .............................6

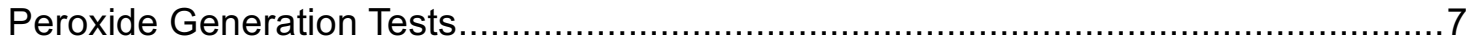

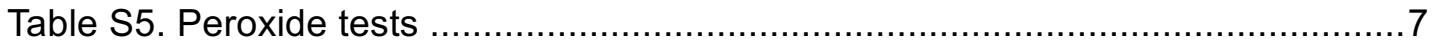

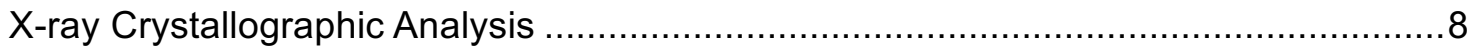

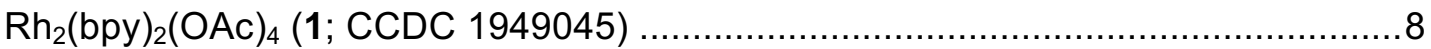

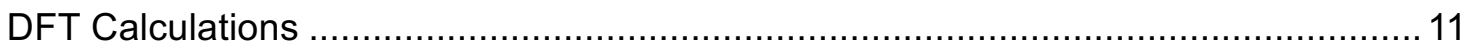

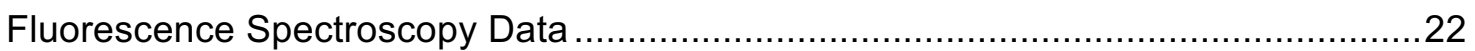

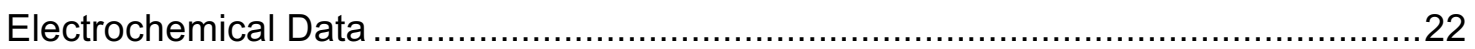

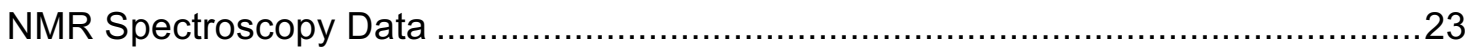

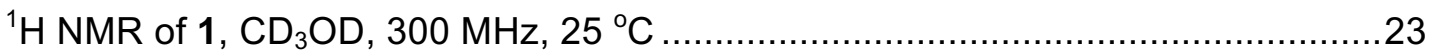

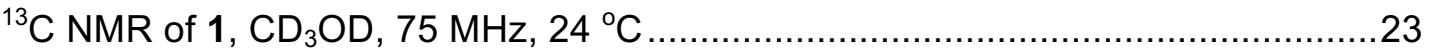

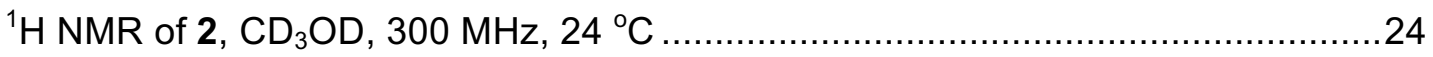

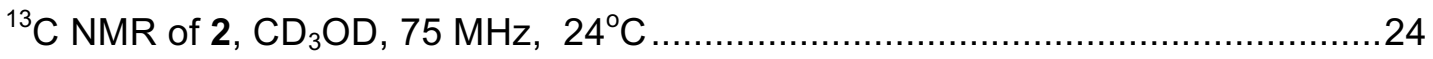

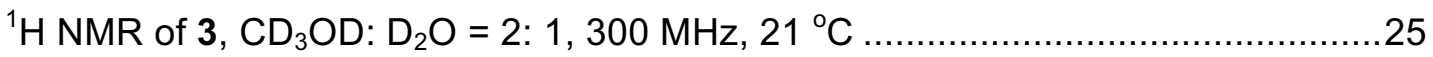

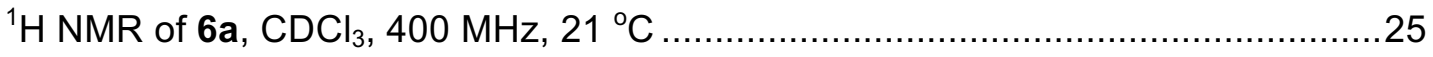

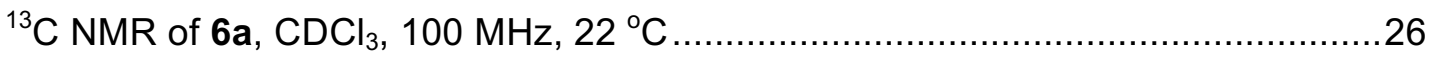

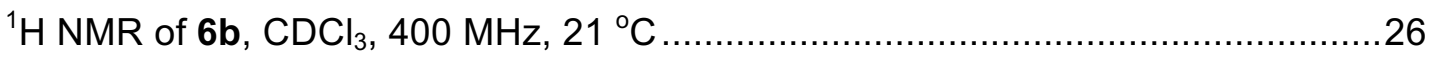

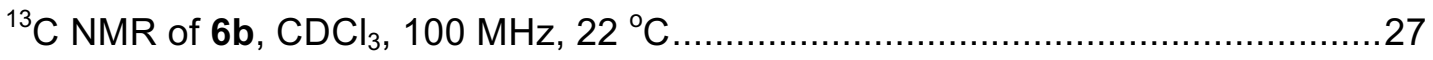

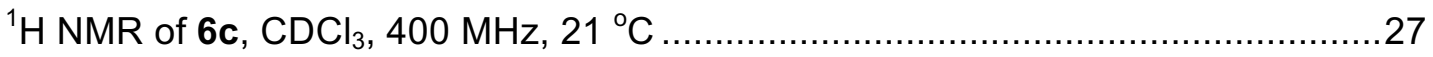

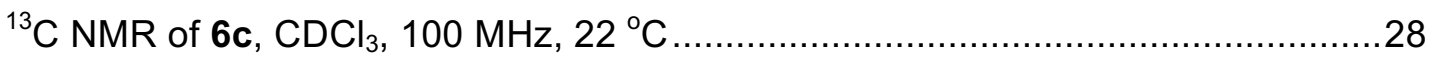

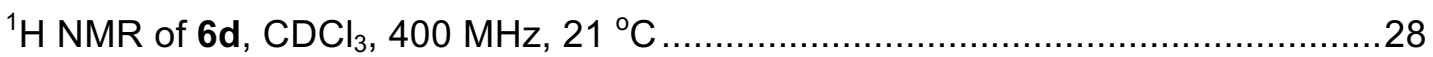

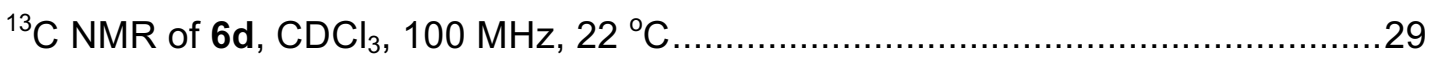

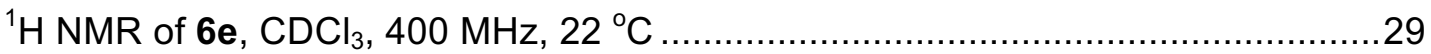

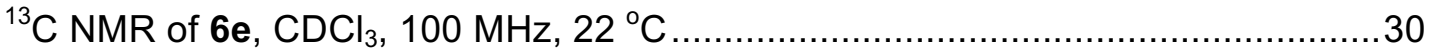




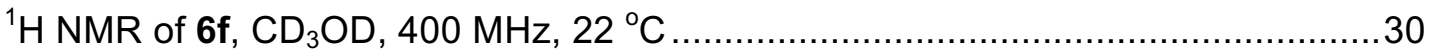

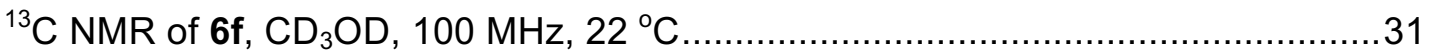

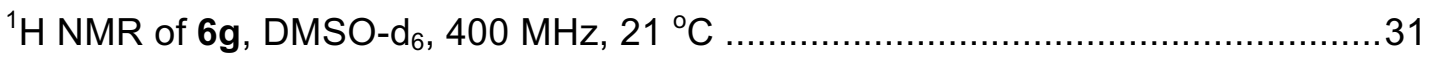

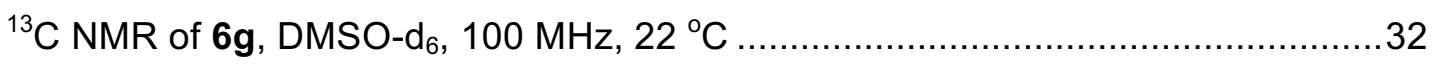

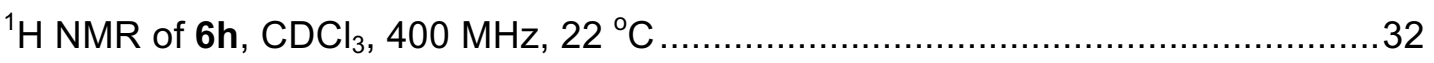

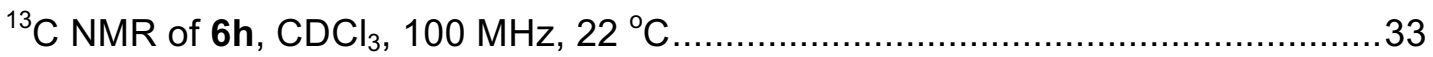

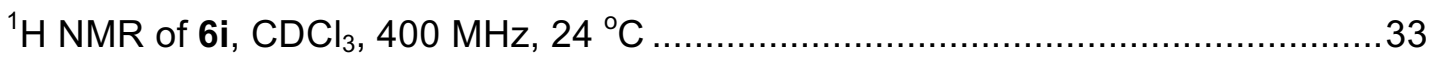

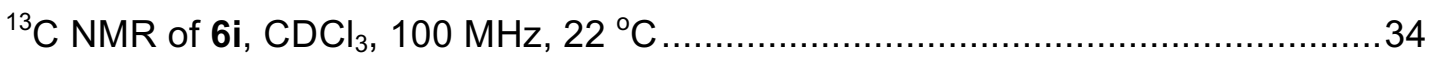

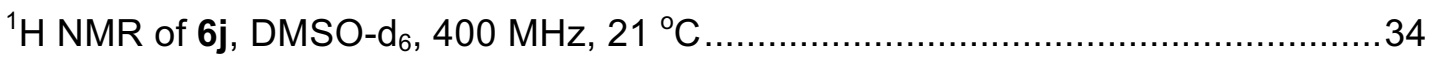

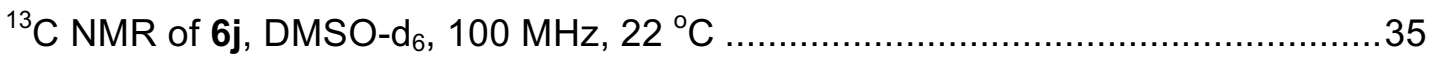

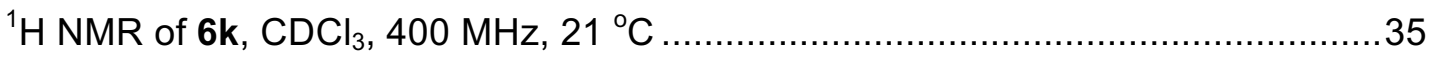

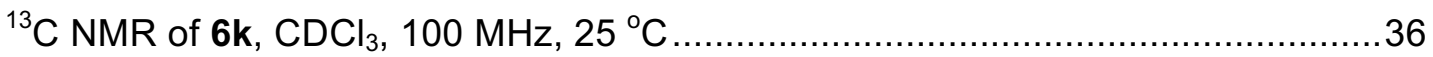

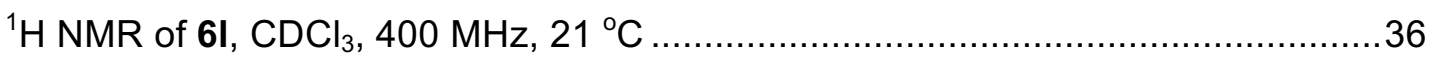

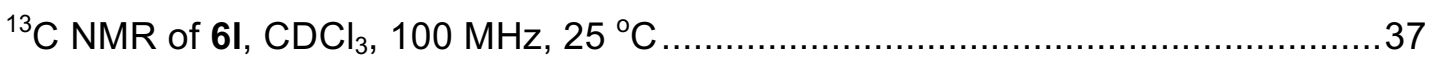

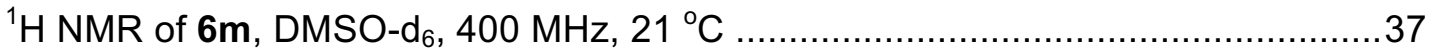

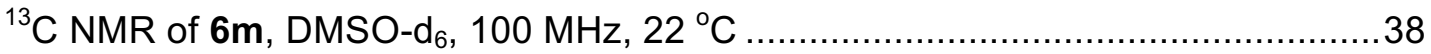

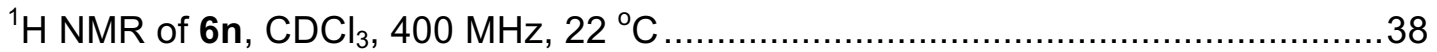

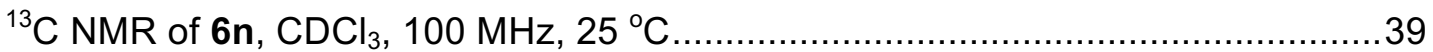

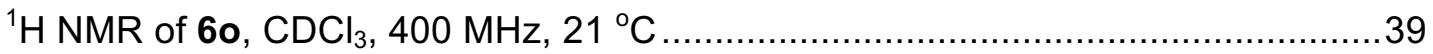

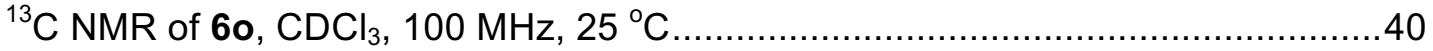

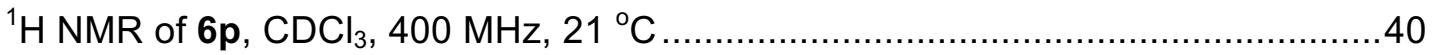

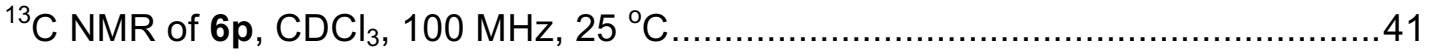

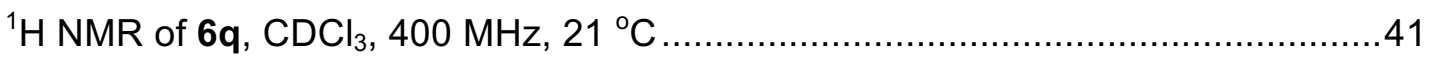

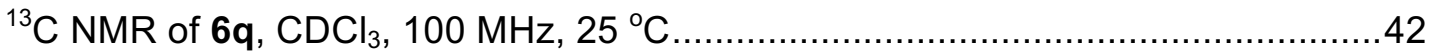

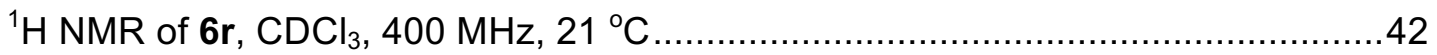

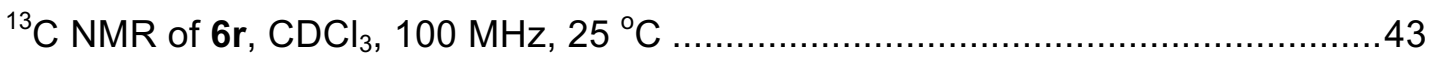

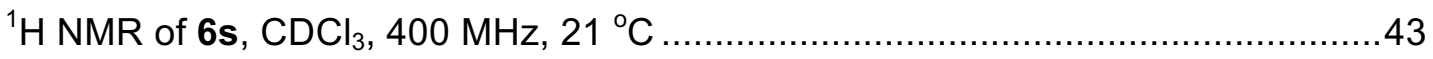

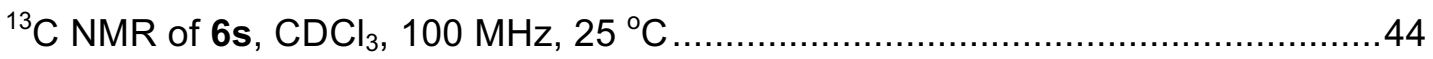

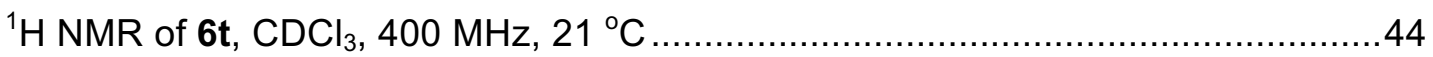

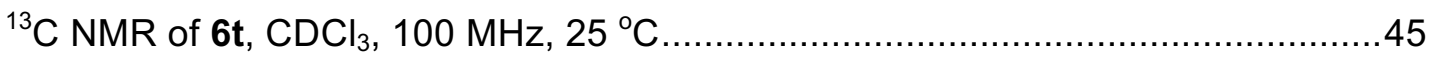

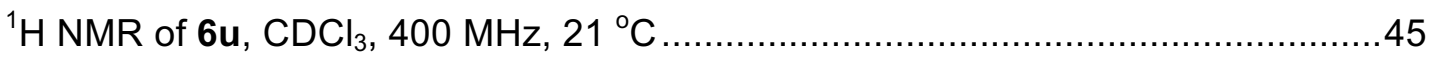

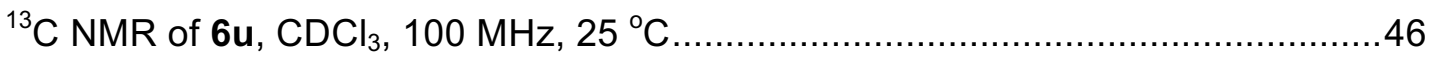

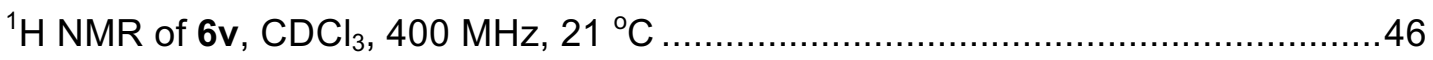

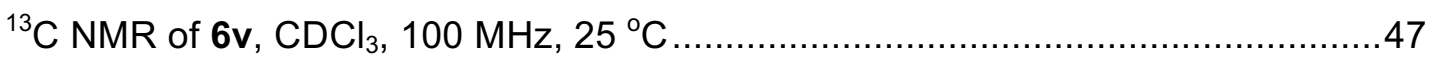




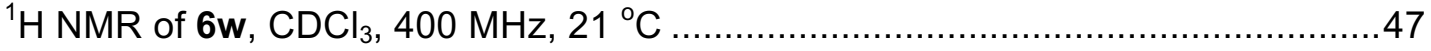

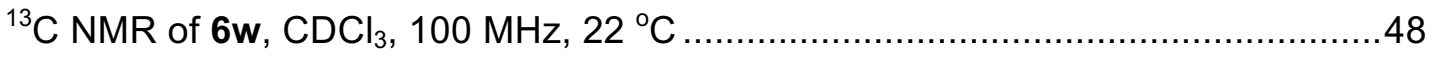

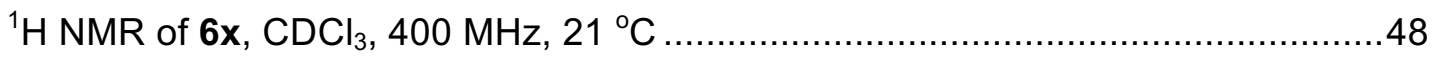

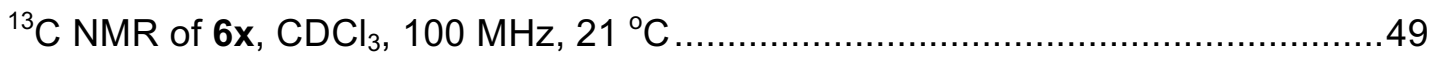

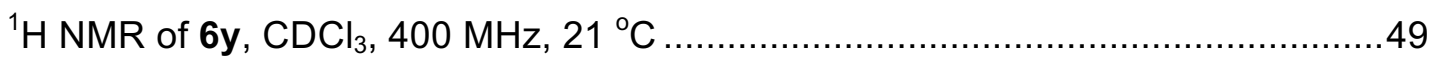

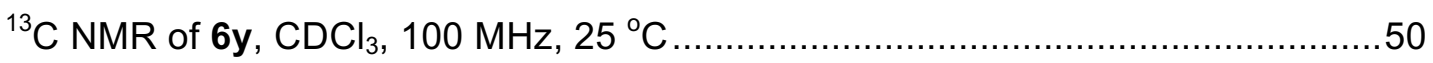

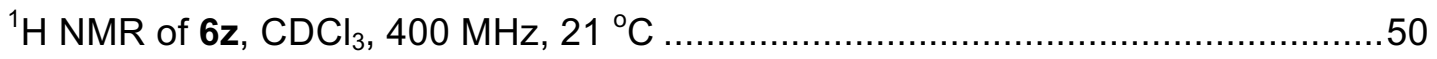

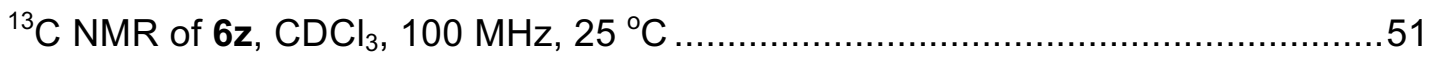

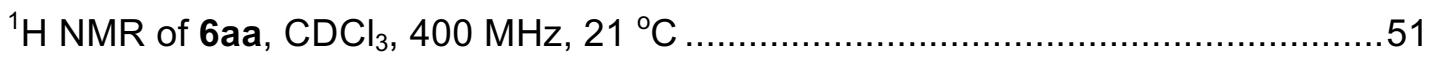

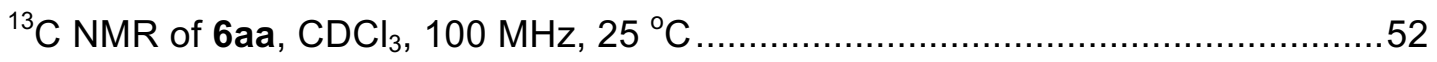

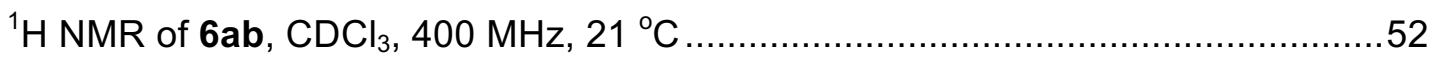

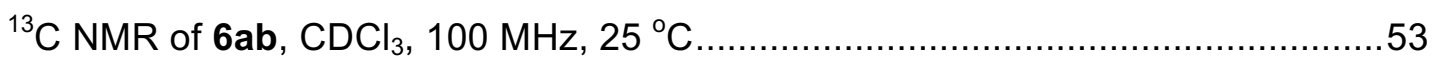

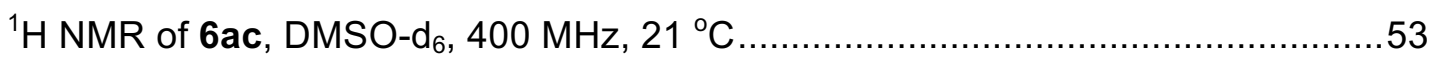

DEPT 135, DEPT 90 and ${ }^{13} \mathrm{C}$ NMR of 6ac, DMSO-d $\mathrm{d}_{6}, 100 \mathrm{MHz}, 2{ }^{\circ} \mathrm{C}$..................54

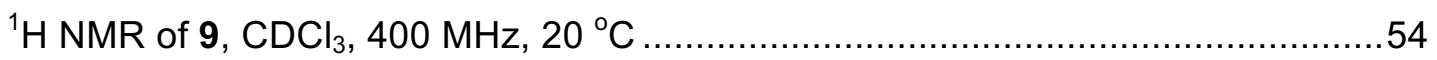

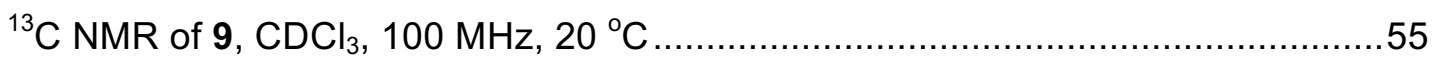

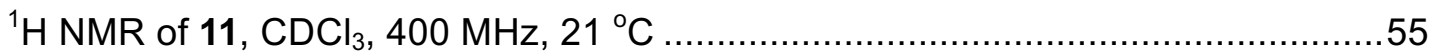

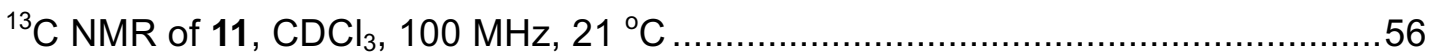

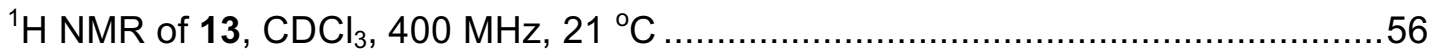

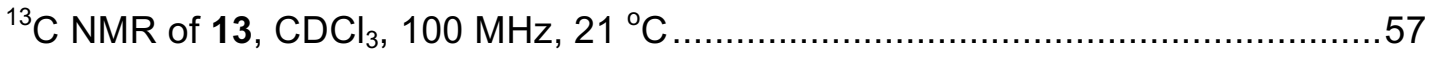




\section{Visible Light Photoreaction Setup}

Reactions were carried out in Pyrex flasks using 10.5 W white, green, or blue LED strips (180×Huga 2835 SMD LEDs, luminous flux: $1300 \mathrm{Im}$ ). No filters were used, and the approximate distance between the light source and the reaction vessel was $\leq 5 \mathrm{~cm}$.
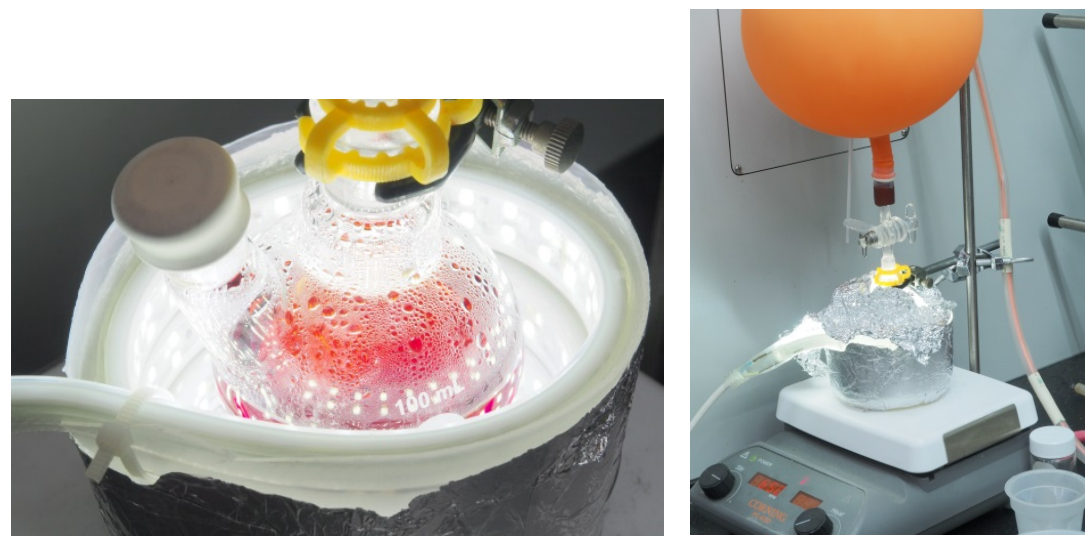

Figure S1. Visible light reaction setup

\section{Experimental Data}

\section{Optimization of Reaction Conditions}

Table S1. Background reaction comparison

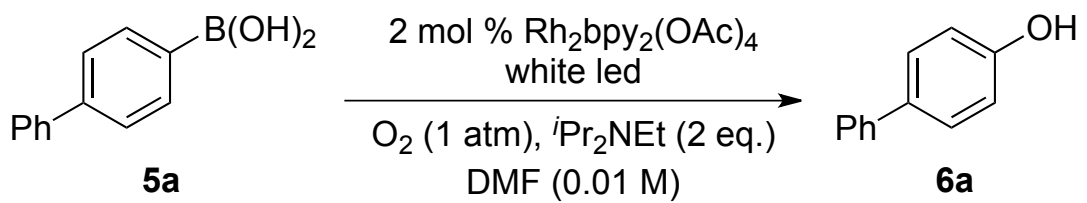

\begin{tabular}{cccccc}
\hline Entry & $h v^{a}$ & DIPEA & Atmosphere $(1 \mathrm{~atm})$ & Time $(\mathrm{h})$ & ${\text { Yield }(\%)^{b}}^{b}$ \\
\hline 1 & + & + & $\mathrm{O}_{2}$ & 18 & 95 \\
2 & - & + & $\mathrm{O}_{2}$ & $>48$ & trace \\
3 & + & - & $\mathrm{O}_{2}$ & $>48$ & trace \\
4 & + & + & $\mathrm{Ar}$ & $>48$ & 0 \\
5 & + & + & $\mathrm{Air}$ & 24 & 65 \\
6 & $+^{c}$ & + & $\mathrm{O}_{2}$ & 18 & 12 \\
7 & $+^{c}{ }^{c}$ & + & $\mathrm{O}_{2}$ & 18 & 87
\end{tabular}

${ }^{a}$ White LED. ${ }^{b}$ Isolated yield. ${ }^{c}$ Green LED (centered at $530 \mathrm{~nm}$ ). ${ }^{d}$ Blue LED (centered at $460 \mathrm{~nm}$ ). 
Table S2. Optimization of reductant.

\begin{tabular}{|c|c|c|c|}
\hline 1 & $\begin{array}{c}2 \mathrm{~mol} \% \\
\mathrm{O}_{2}(1 \\
\mathrm{DN}\end{array}$ & $\begin{array}{l}\mathrm{y}_{2}(\mathrm{OAc})_{4} \\
\frac{\mathrm{d}}{\text { ductant }} \\
1 \mathrm{M})\end{array}$ & $6 a$ \\
\hline Entry & Reductant, eq. & Time (h) & Yield $(\%)^{a}$ \\
\hline 1 & DIPEA, 4 & 18 & 97 \\
\hline 2 & DIPEA, 2 & 18 & 95 \\
\hline 3 & DIPEA, 1.1 & 24 & 91 \\
\hline 4 & $\mathrm{Et}_{3} \mathrm{~N}, 2$ & 36 & 88 \\
\hline 5 & DABCO, 2 & 24 & 69 \\
\hline 6 & Pyridine, 2 & 48 & 87 \\
\hline 7 & $\mathrm{Ph}_{3} \mathrm{P}, 2$ & $>48$ & 29 \\
\hline
\end{tabular}

${ }^{a}$ Isolated Yield.

Table S3. Optimization of solvent.

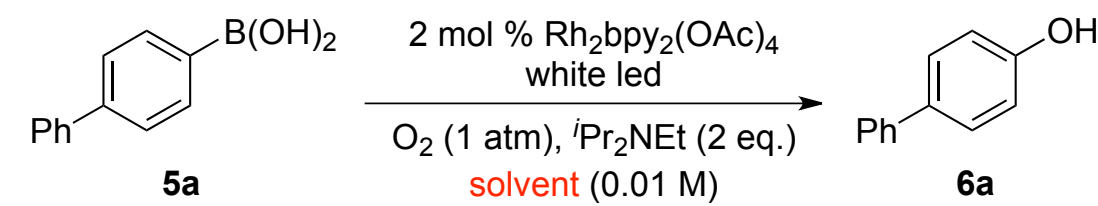

\begin{tabular}{cccc}
\hline Entry & Solvent & Time (h) & Yield (\%) $)^{a}$ \\
\hline 1 & DMF & 18 & 95 \\
2 & $\mathrm{MeCN}$ & 24 & 61 \\
3 & $\mathrm{MeOH}$ & 24 & 50 \\
4 & $i-\mathrm{PrOH}$ & 24 & 54 \\
5 & DMSO & 48 & 60 \\
\hline
\end{tabular}

${ }^{a}$ Isolated Yield. 
Table S4. Optimization of catalyst loading.

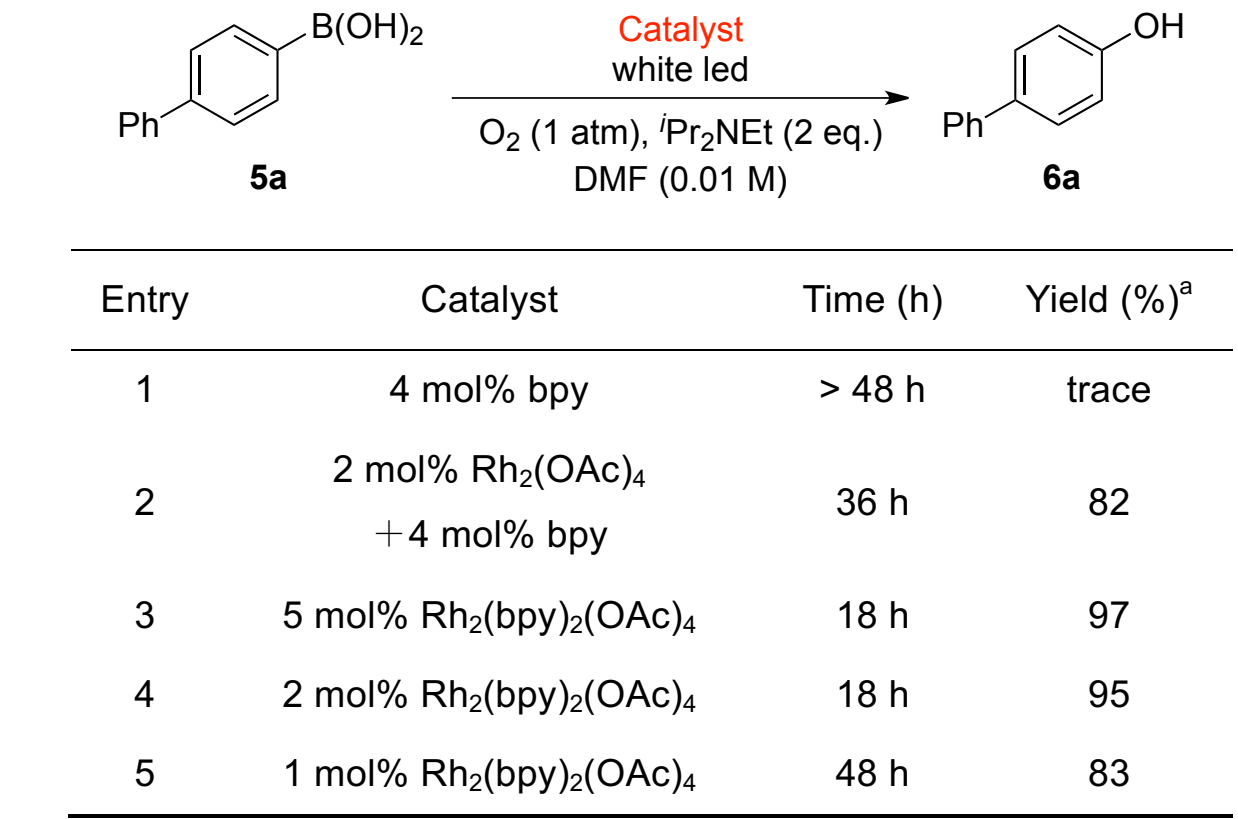

${ }^{a}$ Isolated yield.

\section{Light Source On-Off Experiment (data pertaining to Figure 1)}

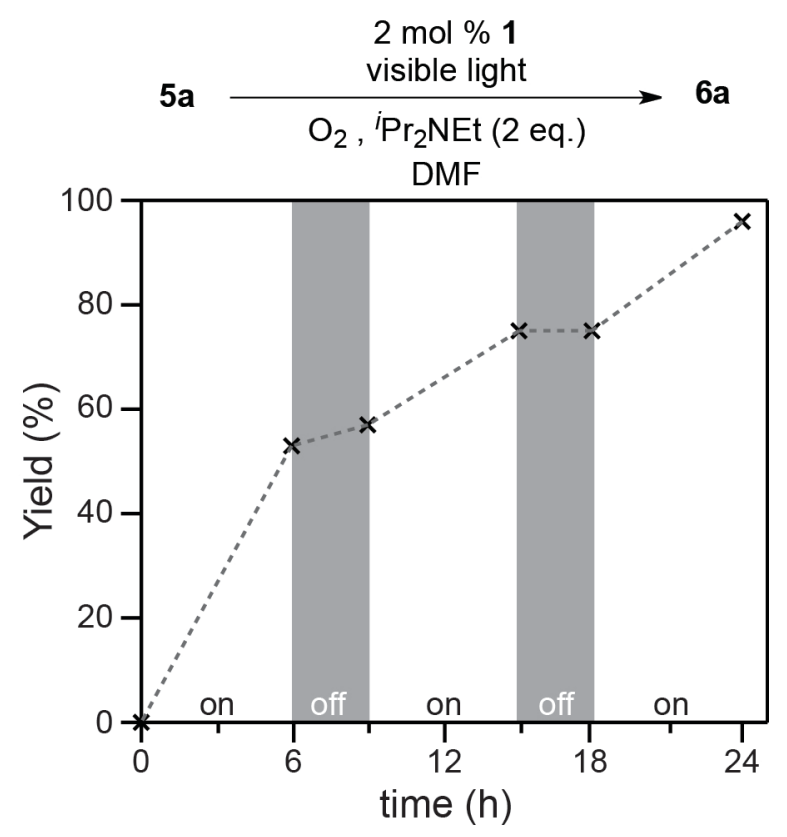

Five identical reactions for the conversion of $\mathbf{5 a}$ to $\mathbf{6 a}$, using the standard conditions as described above, were set up in parallel and exposed to same lighting conditions throughout the duration of the experiment. The light source was switched on and off at 
the time points indicated in the graph above. Individual reactions were quenched at each specified time point, and product was isolated in order to determine the yield.

\section{Peroxide Generation Tests}

Table S5. Peroxide tests

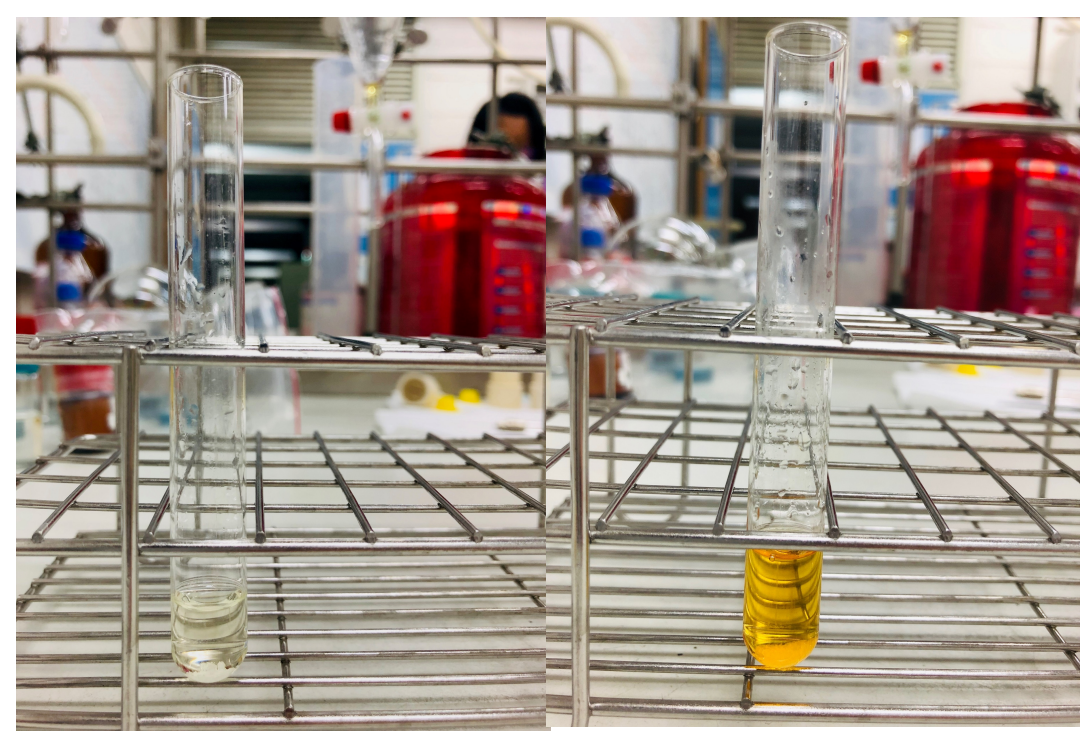

"Negative"

"Positive"

\section{Entry Reaction mixture}

Peroxide Test Result

1 Standard reaction condition using $\mathbf{5 a}$

Positive (yellow)

2

Standard reaction condition without 5 a

Positive (yellow)

3

Standard reaction condition using $\mathbf{5 a}$, without DIPEA

Negative (clear)

After $12 \mathrm{~h}$ of irradiation, $0.3 \mathrm{~mL}$ of the reaction mixture was added to a freshly prepared solution of $0.1 \mathrm{~g}$ of potassium iodide in $1 \mathrm{~mL}$ of glacial acetic acid. A positive test (color change to yellow) indicates the presence of peroxide and/or other reactive oxygen species in the reaction mixture. 


\section{X-ray Crystallographic Analysis}

\section{$\mathrm{Rh}_{2}(\mathrm{bpy})_{2}(\mathrm{OAC})_{4}(1 ; \mathrm{CCDC} 1949045)$}

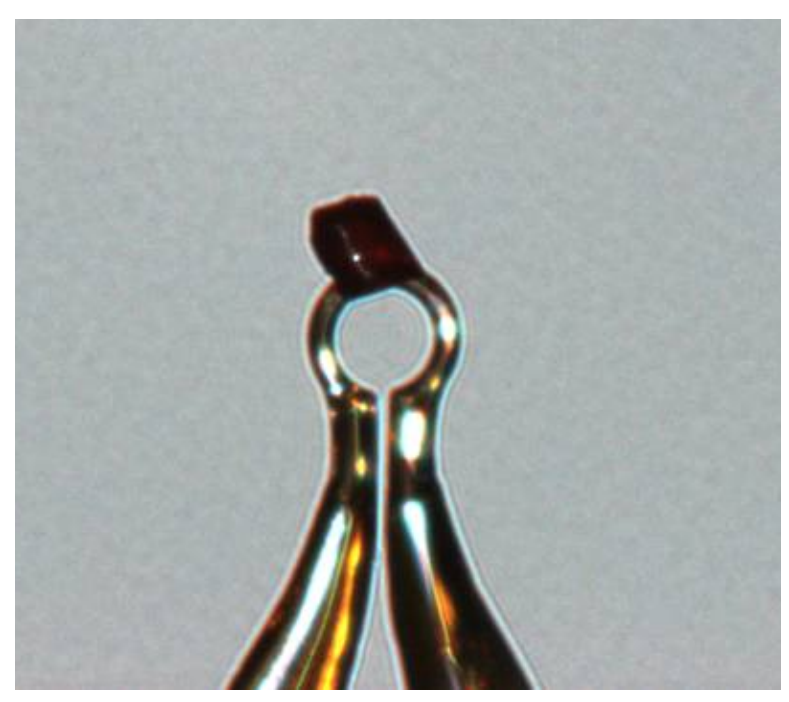

Figure S2. Single crystal of 1 used for X-ray analysis.

Crystals of 1 suitable for X-ray diffraction were grown as follows: $20 \mathrm{mg}$ of 1 was dissolved in $1 \mathrm{~mL}$ of $\mathrm{MeOH}$, and then $0.1 \mathrm{~mL}$ of this solution was added to $1 \mathrm{~mL}$ of DMF. ${ }^{1}$ The resulting solution was mixed thoroughly, filtered to remove any undissolved solids, and transferred to a $4 \mathrm{~mL}$ vial. The vial was then placed, uncapped, into a $20 \mathrm{~mL}$ vial containing $5 \mathrm{~mL}$ of $\mathrm{Et}_{2} \mathrm{O}$. The outer vial was capped, and vapor diffusion of $\mathrm{Et}_{2} \mathrm{O}$ into the $\mathrm{DMF} / \mathrm{MeOH}$ solution resulted in the growth of dark red crystals after 24 hours.

A single crystal of 1 was mounted on a polymer loop using Paratone-N oil, and transferred to an Agilent SuperNova diffractometer using mirror-monochromated Mo K $\alpha$ radiation. The sample was held at $100 \mathrm{~K}$ during the experiment. Data collection, integration, scaling (ABSPACK) and absorption correction (numeric analytical methods ${ }^{2}$ ) were performed in CrysAlisPro. ${ }^{3}$ Structure solution was performed using ShelXT, ${ }^{4}$ and subsequent refinement was performed by full-matrix least-squares on $F^{2}$ in ShelXL,

\footnotetext{
${ }^{1}$ While complex 1 is readily soluble in the reaction mixture used for catalysis, it exhibits significantly lower solubility in DMF alone. We have found that a small amount of $\mathrm{MeOH}$ as cosolvent best facilitates crystallization from DMF.

${ }^{2}$ Clark. R. C.; Reid, J. S. Acta Cryst. A51 (1995), 887-897.

${ }^{3}$ Version 1.171.37.35 (2014). Oxford Diffraction /Agilent Technologies UK Ltd, Yarnton, England.

${ }^{4}$ Sheldrick, G. M. Acta Cryst. A71 (2015), 3-8.
} 
using the Olex2 interface. ${ }^{5}$ Non-hydrogen atoms were refined anisotropically, and hydrogen atoms were allowed to ride on their respective atoms. PLATON ${ }^{6}$ was used for SQUEEZE ${ }^{7}$ due to the presence of disordered solvent molecules. Graphics were prepared using CrystalMaker. ${ }^{8}$ Crystal details, as well as parameters for data collection and refinement, are given in Table S6.

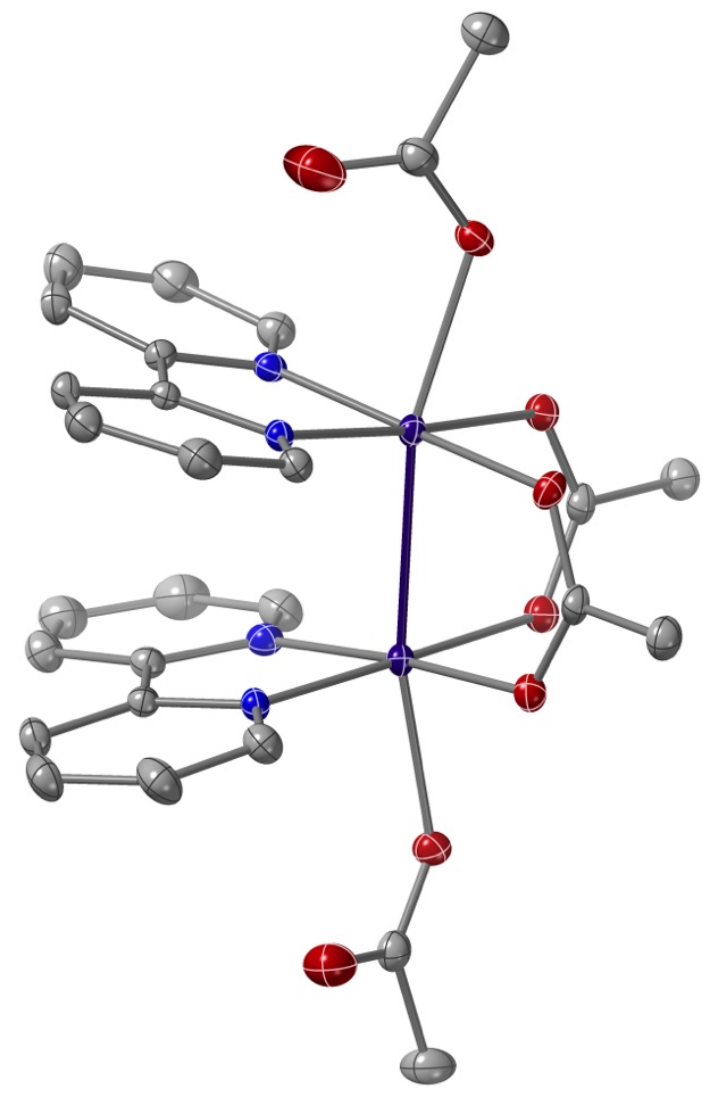

Figure S3. X-ray structure of 1 , plotted with $50 \%$ probability ellipsoids ( $\mathrm{H}$ atoms omitted for clarity).

\footnotetext{
${ }^{5}$ Dolomanov, O. V.; Bourhis, L. J.; Gildea, R. J.; Howard, J. A. K.; Puschmann, H. J. Appl. Cryst. 42 (2009), 339-341.

${ }^{6}$ Spek, A. Acta Cryst. D65 (2009), 148-155.

${ }^{7}$ Van der Sluis, P.; Spek, A. L. Acta Cryst. A46 (1990), 194-201.

${ }^{8}$ CrystalMaker Software Ltd, Oxford, England (www.crystalmaker.com).
} 
Table S6. Experimental details for SCXRD analysis of 1

\begin{tabular}{|c|c|}
\hline \multicolumn{2}{|l|}{ Crystal data } \\
\hline Chemical formula & $\mathrm{C}_{28} \mathrm{H}_{28} \mathrm{~N}_{4} \mathrm{O}_{8} \mathrm{Rh}_{2}$ \\
\hline$M_{\mathrm{r}}$ & 754.36 \\
\hline $\begin{array}{l}\text { Crystal system, } \\
\text { space group }\end{array}$ & Monoclinic, $P 2_{1} / c$ \\
\hline Temperature (K) & 100 \\
\hline$a, b, c(\AA)$ & $9.7654(2), 17.1242(3), 17.5385(3)$ \\
\hline$\beta\left(^{\circ}\right)$ & $105.677(2)$ \\
\hline$V\left(\AA^{3}\right)$ & $2823.77(9)$ \\
\hline$Z$ & 4 \\
\hline Radiation type & Mo $K \alpha$ \\
\hline$\mu\left(\mathrm{mm}^{-1}\right)$ & 1.23 \\
\hline \multicolumn{2}{|l|}{ Data collection } \\
\hline Diffractometer & SuperNova, Dual, Cu at zero, EosS2 \\
\hline $\begin{array}{l}\text { Absorption } \\
\text { correction }\end{array}$ & $\begin{array}{l}\text { Multi-scan } \\
\text { CrysAlis PRO 1.171.38.46 (Rigaku Oxford Diffraction, 2015) } \\
\text { Empirical absorption correction using spherical harmonics, } \\
\text { implemented in SCALE3 ABSPACK scaling algorithm. }\end{array}$ \\
\hline$T_{\min }, T_{\max }$ & $0.906,1.000$ \\
\hline $\begin{array}{l}\text { No. of measured, } \\
\text { independent and } \\
\text { observed }[I \quad> \\
2 \sigma(I)] \text { reflections }\end{array}$ & $51946,7260,6433$ \\
\hline$R_{\text {int }}$ & 0.054 \\
\hline$(\sin \theta / \lambda)_{\max }\left(\AA^{-1}\right)$ & 0.695 \\
\hline \multicolumn{2}{|l|}{ Refinement } \\
\hline $\begin{array}{l}R\left[F^{2}>2 \sigma\left(F^{2}\right)\right] \\
w R\left(F^{2}\right), S\end{array}$ & $0.036,0.075,1.11$ \\
\hline No. of reflections & 7260 \\
\hline No. of parameters & 383 \\
\hline $\mathrm{H}$-atom treatment & $\mathrm{H}$-atom parameters constrained \\
\hline$\Delta \rho_{\max }, \Delta \rho_{\min }\left(\mathrm{e} \AA^{-3}\right)$ & $0.58,-0.74$ \\
\hline
\end{tabular}

Computer programs: CrysAlis PRO 1.171.38.46 (Rigaku OD, 2015), SHELXT 2014/5 (Sheldrick, 2014), SHELXL (Sheldrick, 2015), Olex2 (Dolomanov et al., 2009). 


\section{DFT Calculations}

Density functional theory (DFT) calculations were performed using the Gaussian 16 program package,${ }^{9}$ and the GaussView 6 interface. ${ }^{10}$ Geometry optimization was carried out using the atomic coordinates from the crystal structures of 1 and 7 as a starting point. Calculations were performed using the B3PW91 functional and the LanL2DZ basis set. Frequency calculations on the geometry-optimized structures using the same method resulted in no imaginary frequencies, confirming that the geometries obtained are indeed local minima.

Time-dependent DFT (TD-DFT) calculations were performed on the geometry-optimized structure of 1 using B3PW91/LanL2DZ and the polarizable continuum model with DMF as the solvent. Analysis of simulated UV-vis spectra, molecular orbitals, and atomic contributions to the frontier molecular orbitals (Mulliken contribution) were carried out using Chemissian. ${ }^{11}$ Molecular orbital visualizations were generated using an isosurface value of 0.03 .

\footnotetext{
${ }^{9}$ Gaussian 16, Revision B.01, M. J. Frisch, G. W. Trucks, H. B. Schlegel, G. E. Scuseria, M. A. Robb, J. R. Cheeseman, G. Scalmani, V. Barone, G. A. Petersson, H. Nakatsuji, X. Li, M. Caricato, A. V. Marenich, J. Bloino, B. G. Janesko, R. Gomperts, B. Mennucci, H. P. Hratchian, J. V. Ortiz, A. F. Izmaylov, J. L. Sonnenberg, D. Williams-Young, F. Ding, F. Lipparini, F. Egidi, J. Goings, B. Peng, A. Petrone, T. Henderson, D. Ranasinghe, V. G. Zakrzewski, J. Gao, N. Rega, G. Zheng, W. Liang, M. Hada, M. Ehara, K. Toyota, R. Fukuda, J. Hasegawa, M. Ishida, T. Nakajima, Y. Honda, O. Kitao, H. Nakai, T. Vreven, K. Throssell, J. A. Montgomery, Jr., J. E. Peralta, F. Ogliaro, M. J. Bearpark, J. J. Heyd, E. N. Brothers, K. N. Kudin, V. N. Staroverov, T. A. Keith, R. Kobayashi, J. Normand, K. Raghavachari, A. P. Rendell, J. C. Burant, S. S. lyengar, J. Tomasi, M. Cossi, J. M. Millam, M. Klene, C. Adamo, R. Cammi, J. W. Ochterski, R. L. Martin, K. Morokuma, O. Farkas, J. B. Foresman, and D. J. Fox, Gaussian, Inc., Wallingford CT, 2016.

${ }^{10}$ GaussView, Version 6, Roy Dennington, Todd A. Keith, and John M. Millam, Semichem Inc., Shawnee Mission, KS, 2016.

${ }^{11}$ Chemissian version 4.60, @ Skripnikov Leonid 2005-2018, www.chemissian.com
} 
Table S7. Cartesian Coordinates for the Optimized Structure of 1

\begin{tabular}{|c|c|c|c|}
\hline Symbol & $x$ & $Y$ & Z \\
\hline $\mathrm{Rh}$ & -1.41221 & -0.601 & -0.10185 \\
\hline $\mathrm{Rh}$ & 1.22359 & -0.89257 & -0.1747 \\
\hline 0 & 0.853799 & -1.9564 & -1.92042 \\
\hline 0 & -1.38275 & -2.15133 & -1.50399 \\
\hline 0 & 0.827448 & -2.63457 & 0.904976 \\
\hline 0 & -1.32862 & -2.04905 & 1.377691 \\
\hline 0 & 3.251455 & -1.6432 & -0.31977 \\
\hline 0 & 4.387549 & 0.335159 & -0.01648 \\
\hline 0 & -3.5602 & -0.81406 & -0.20492 \\
\hline 0 & -4.06202 & -0.05483 & 1.911935 \\
\hline $\mathrm{N}$ & -1.44525 & 0.964579 & 1.128936 \\
\hline $\mathrm{N}$ & -1.6065 & 0.860276 & -1.44348 \\
\hline $\mathrm{N}$ & 1.690888 & 0.211565 & 1.422171 \\
\hline $\mathrm{N}$ & 1.630326 & 0.830434 & -1.08864 \\
\hline C & -1.4824 & 0.861887 & 2.478568 \\
\hline $\mathrm{H}$ & -1.48772 & -0.14563 & 2.869827 \\
\hline C & -1.54928 & 1.99406 & 3.29111 \\
\hline $\mathrm{H}$ & -1.57757 & 1.87445 & 4.368193 \\
\hline C & -1.62026 & 3.26494 & 2.694742 \\
\hline $\mathrm{H}$ & -1.68889 & 4.159181 & 3.306712 \\
\hline C & -1.6328 & 3.3626 & 1.299822 \\
\hline $\mathrm{H}$ & -1.72489 & 4.329916 & 0.818338 \\
\hline C & -1.54758 & 2.192242 & 0.52881 \\
\hline C & -1.65237 & 2.133496 & -0.93036 \\
\hline C & -1.87232 & 3.234434 & -1.77281 \\
\hline $\mathrm{H}$ & -1.90756 & 4.236338 & -1.35996 \\
\hline C & -2.07324 & 3.028244 & -3.14186 \\
\hline $\mathrm{H}$ & -2.25006 & 3.870793 & -3.80311 \\
\hline C & -2.07096 & 1.713542 & -3.64099 \\
\hline $\mathrm{H}$ & -2.25732 & 1.507687 & -4.68944 \\
\hline C & -1.83948 & 0.653793 & -2.76245 \\
\hline $\mathrm{H}$ & -1.86285 & -0.38132 & -3.07725 \\
\hline C & 1.825532 & -0.27794 & 2.676457 \\
\hline $\mathrm{H}$ & 1.611657 & -1.332 & 2.796043 \\
\hline C & 2.250645 & 0.526814 & 3.736098 \\
\hline $\mathrm{H}$ & 2.355242 & 0.096023 & 4.725983 \\
\hline C & 2.545812 & 1.877652 & 3.490927 \\
\hline $\mathrm{H}$ & 2.87957 & 2.525613 & 4.29537 \\
\hline C & 2.430918 & 2.371873 & 2.186859 \\
\hline $\mathrm{H}$ & 2.694576 & 3.400163 & 1.968211 \\
\hline C & 2.018152 & 1.516818 & 1.157329 \\
\hline C & 1.97655 & 1.868962 & -0.26303 \\
\hline C & 2.328067 & 3.115784 & -0.79576 \\
\hline $\mathrm{H}$ & 2.608179 & 3.930254 & -0.13758 \\
\hline
\end{tabular}

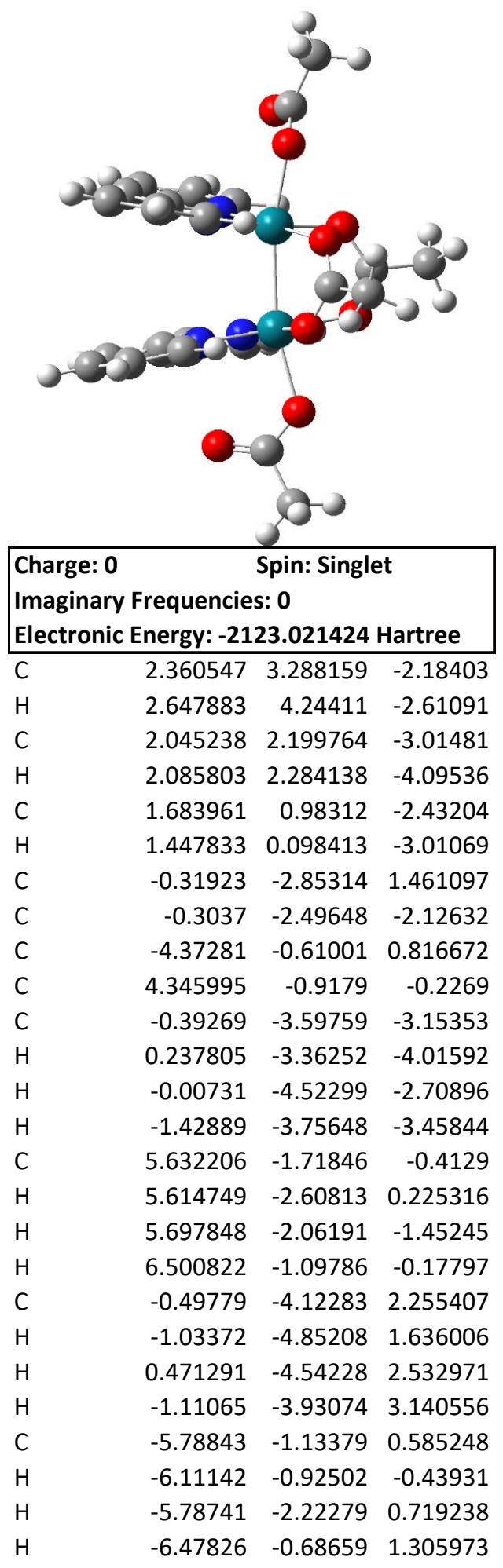


Table S8. Selected Frontier MOs for 1

\begin{tabular}{|c|c|c|}
\hline $\begin{array}{c}\text { MO \# } \\
\text { (Energy, eV) }\end{array}$ & $\begin{array}{l}\text { Atomic } \\
\text { Contributions }\end{array}$ & $\begin{array}{c}\text { Visualization } \\
\text { (isosurface value } 0.03 \text { ) }\end{array}$ \\
\hline $\begin{array}{c}\text { 166, LUMO+4 } \\
(-1.526)\end{array}$ & $\begin{array}{c}\text { C(bpy): } 82 \% \\
\text { N(bpy): } 15 \% \\
\text { Rh: } 2 \%\end{array}$ & \\
\hline $\begin{array}{c}\text { 165, LUMO+3 } \\
(-1.564)\end{array}$ & $\begin{array}{c}\text { Rh: } 71 \% \\
\text { O(axial): } 11 \% \\
\text { N(bpy): } 7 \% \\
\text { C(bpy): } 4 \% \\
\text { O(bridging): } 4 \%\end{array}$ & \\
\hline $\begin{array}{c}\text { 164, LUMO+2 } \\
(-1.705)\end{array}$ & $\begin{array}{c}\text { C(bpy): } 90 \% \\
\text { N(bpy): } 7 \% \\
\text { Rh: } 2 \%\end{array}$ & \\
\hline
\end{tabular}




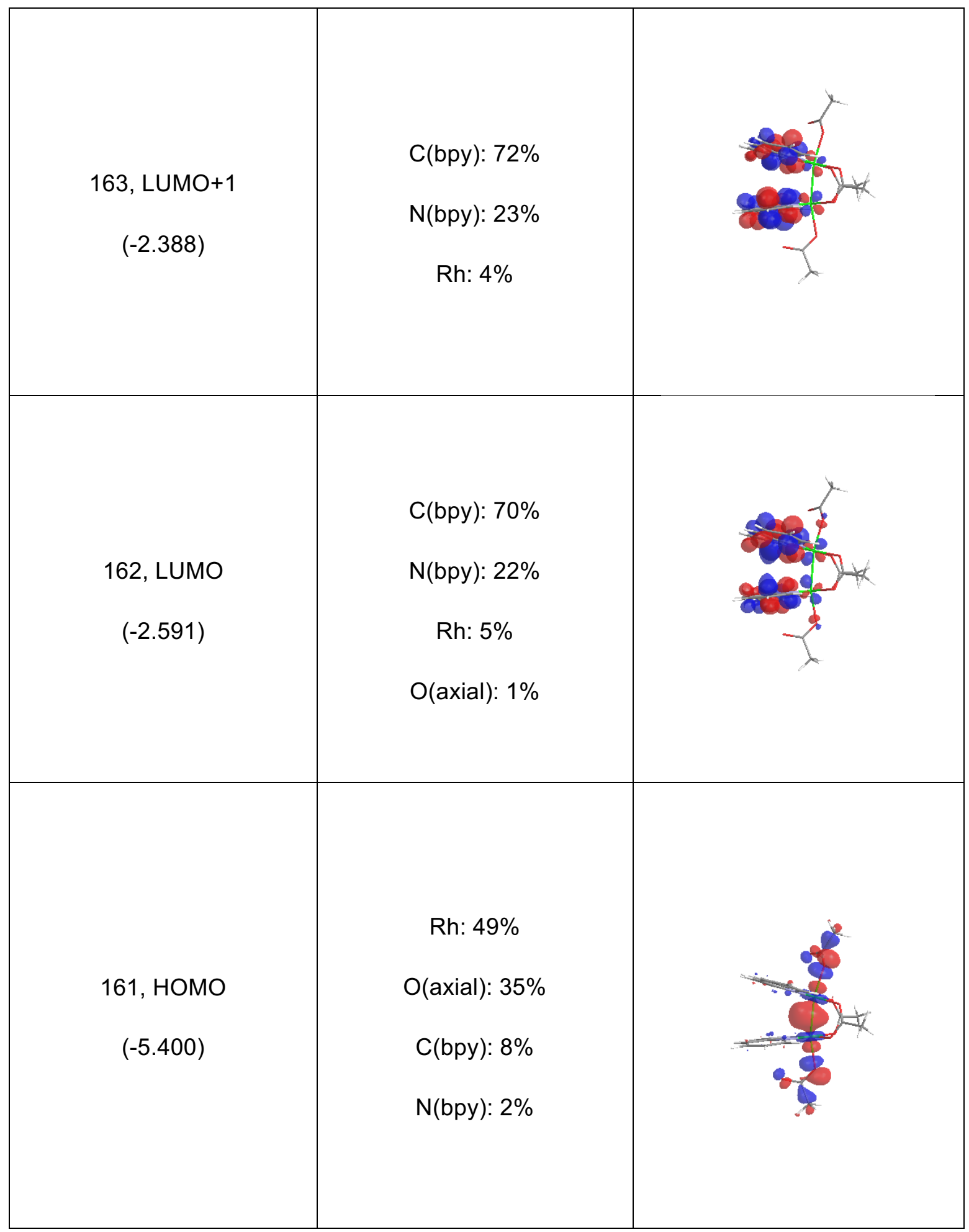




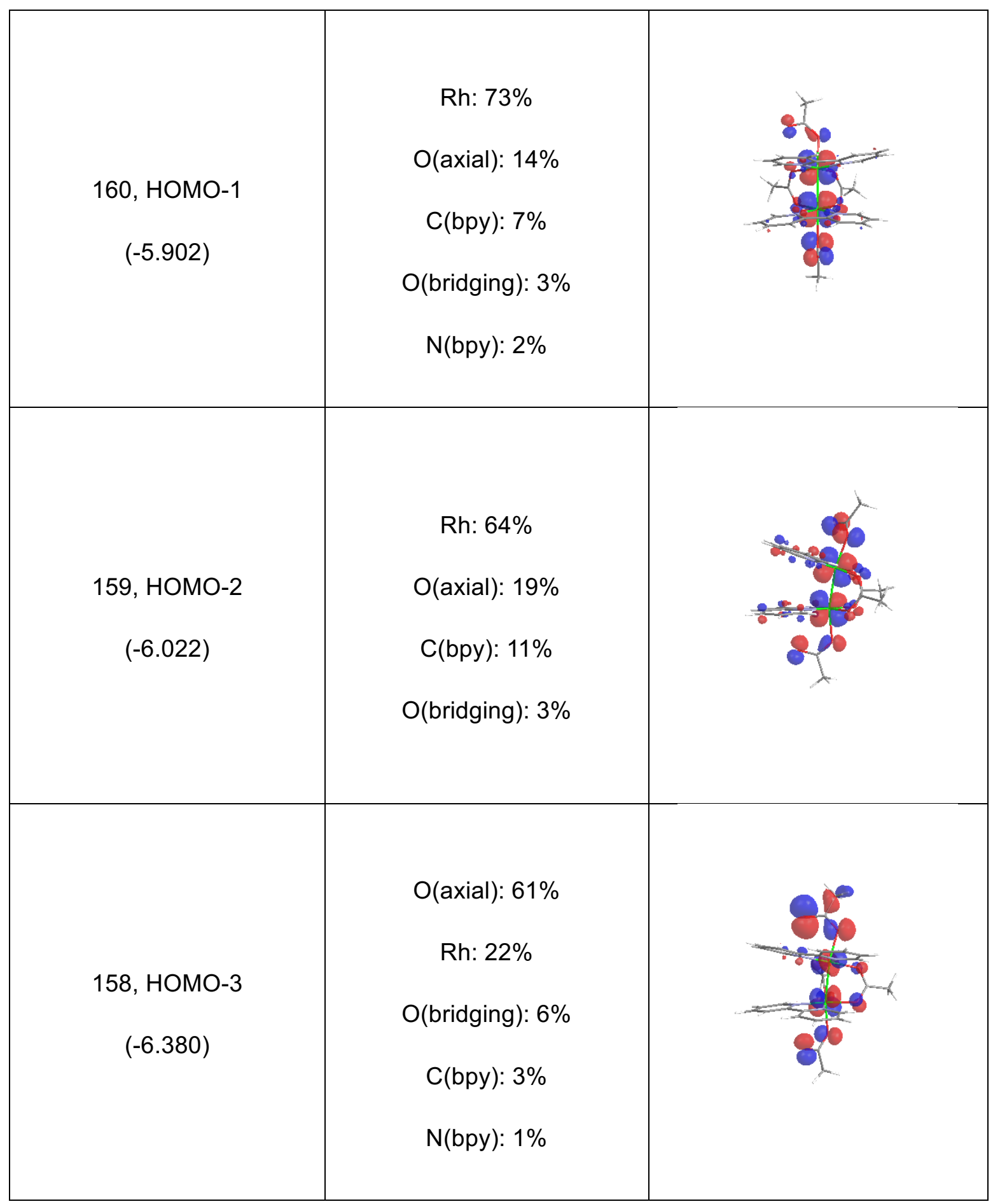




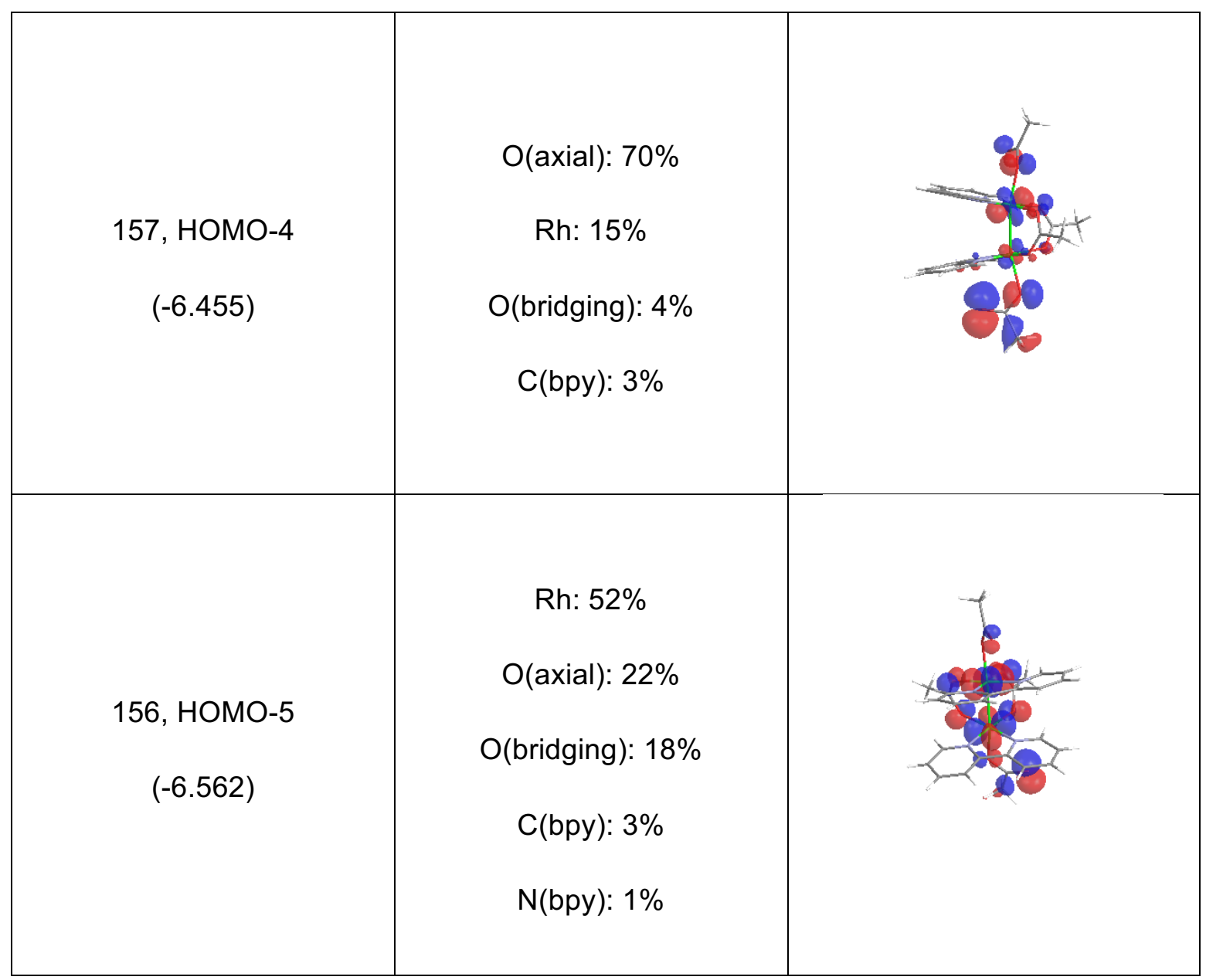


Table S9. Cartesian Coordinates for the Optimized Structure of 7

\begin{tabular}{|c|c|c|c|}
\hline Symbol & $x$ & $\mathbf{Y}$ & Z \\
\hline $\mathrm{Rh}$ & 1.303175 & -0.74411 & -0.04689 \\
\hline $\mathrm{Rh}$ & -1.30329 & -0.74397 & 0.046895 \\
\hline $\mathrm{N}$ & 1.660075 & 0.727867 & 1.287546 \\
\hline C & 1.786818 & 0.538591 & 2.621395 \\
\hline C & 2.119122 & 1.589973 & 3.481012 \\
\hline C & 2.32546 & 2.869668 & 2.943003 \\
\hline C & 2.216117 & 3.055209 & 1.55842 \\
\hline C & 1.89031 & 1.964466 & 0.740904 \\
\hline C & 1.840046 & 2.003057 & -0.72741 \\
\hline C & 2.108079 & 3.136242 & -1.50718 \\
\hline C & 2.12414 & 3.022239 & -2.90375 \\
\hline C & 1.882964 & 1.771117 & -3.49227 \\
\hline C & 1.608591 & 0.675269 & -2.66823 \\
\hline $\mathrm{N}$ & 1.572568 & 0.795744 & -1.32097 \\
\hline $\mathrm{N}$ & -1.57247 & 0.795933 & 1.32097 \\
\hline C & -1.60851 & 0.675463 & 2.668226 \\
\hline C & -1.88273 & 1.771351 & 3.492267 \\
\hline C & -2.12372 & 3.022507 & 2.903746 \\
\hline C & -2.10765 & 3.136507 & 1.50718 \\
\hline C & -1.83978 & 2.003284 & 0.727407 \\
\hline C & -1.89005 & 1.964693 & -0.74091 \\
\hline C & -2.21569 & 3.055482 & -1.55843 \\
\hline C & -2.32507 & 2.869949 & -2.94301 \\
\hline C & -2.11893 & 1.590217 & -3.48101 \\
\hline C & -1.78678 & 0.53879 & -2.62139 \\
\hline $\mathrm{N}$ & -1.66001 & 0.728057 & -1.28754 \\
\hline 0 & 1.181136 & -2.26945 & 1.360312 \\
\hline C & 0.059048 & -2.7025 & 1.839237 \\
\hline 0 & -1.08666 & -2.19154 & 1.52051 \\
\hline C & 0.086802 & -3.85771 & 2.801545 \\
\hline 0 & 1.086355 & -2.19165 & -1.5205 \\
\hline C & -0.0594 & -2.70251 & -1.83921 \\
\hline 0 & -1.18145 & -2.26933 & -1.3603 \\
\hline C & -0.08727 & -3.85774 & -2.80148 \\
\hline $\mathrm{N}$ & 3.517054 & -1.12219 & -0.12262 \\
\hline C & 4.66027 & -1.36782 & -0.14482 \\
\hline C & 6.082207 & -1.67827 & -0.17022 \\
\hline $\mathrm{N}$ & -3.51719 & -1.12183 & 0.122611 \\
\hline C & -4.66041 & -1.36743 & 0.144786 \\
\hline C & -6.08236 & -1.67783 & 0.170141 \\
\hline $\mathrm{H}$ & 1.63092 & -0.46931 & 2.982027 \\
\hline $\mathrm{H}$ & 2.211523 & 1.401348 & 4.54392 \\
\hline $\mathrm{H}$ & 2.578486 & 3.705319 & 3.586087 \\
\hline $\mathrm{H}$ & 2.395254 & 4.031767 & 1.126212 \\
\hline
\end{tabular}

\begin{tabular}{|c|c|c|c|}
\hline \multirow{2}{*}{\multicolumn{4}{|c|}{ 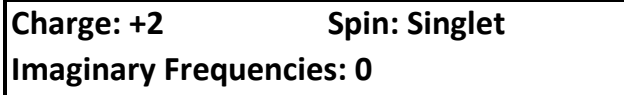 }} \\
\hline & & & \\
\hline \multicolumn{4}{|c|}{ Electronic Energy: -1931.418364 Hartree } \\
\hline $\mathrm{H}$ & 2.313504 & 4.090639 & -1.03853 \\
\hline $\mathrm{H}$ & 2.332442 & 3.890934 & -3.51847 \\
\hline $\mathrm{H}$ & 1.903933 & 1.638382 & -4.56736 \\
\hline $\mathrm{H}$ & 1.426397 & -0.31377 & -3.06777 \\
\hline $\mathrm{H}$ & -1.42647 & -0.3136 & 3.067766 \\
\hline $\mathrm{H}$ & -1.90371 & 1.638617 & 4.567358 \\
\hline $\mathrm{H}$ & -2.3319 & 3.891232 & 3.518468 \\
\hline $\mathrm{H}$ & -2.31294 & 4.090934 & 1.038526 \\
\hline $\mathrm{H}$ & -2.39468 & 4.032071 & -1.12622 \\
\hline $\mathrm{H}$ & -2.57796 & 3.705635 & -3.5861 \\
\hline $\mathrm{H}$ & -2.21136 & 1.401602 & -4.54392 \\
\hline $\mathrm{H}$ & -1.63104 & -0.46913 & -2.98201 \\
\hline $\mathrm{H}$ & 1.019842 & -3.86151 & 3.368836 \\
\hline $\mathrm{H}$ & -0.76923 & -3.81242 & 3.477891 \\
\hline $\mathrm{H}$ & 0.026924 & -4.7956 & 2.235932 \\
\hline $\mathrm{H}$ & -1.02046 & -3.86168 & -3.36852 \\
\hline $\mathrm{H}$ & 0.768577 & -3.81235 & -3.47807 \\
\hline $\mathrm{H}$ & -0.02709 & -4.79562 & -2.23587 \\
\hline $\mathrm{H}$ & 6.230332 & -2.76224 & -0.17744 \\
\hline $\mathrm{H}$ & 6.545525 & -1.25335 & -1.06556 \\
\hline $\mathrm{H}$ & 6.574892 & -1.26141 & 0.713213 \\
\hline $\mathrm{H}$ & -6.54572 & -1.25278 & 1.065396 \\
\hline $\mathrm{H}$ & -6.57498 & -1.26106 & -0.71338 \\
\hline $\mathrm{H}$ & -6.23052 & -2.7618 & 0.177494 \\
\hline
\end{tabular}


Table S10. Selected Frontier MOs for 7

\begin{tabular}{|c|c|c|}
\hline $\begin{array}{c}\text { MO \# } \\
\text { (Energy, eV) }\end{array}$ & $\begin{array}{l}\text { Atomic } \\
\text { Contributions }\end{array}$ & $\begin{array}{c}\text { Visualization } \\
\text { (isosurface value } 0.03 \text { ) }\end{array}$ \\
\hline $\begin{array}{c}\text { 154, LUMO+2 } \\
(-2.629)\end{array}$ & $\begin{array}{c}\text { Rh: } 76 \% \\
\text { N(bpy): } 7 \% \\
\text { N(MeCN): } 7 \% \\
\text { O(bridging): } 4 \% \\
\text { C(bpy): } 3 \%\end{array}$ & \\
\hline $\begin{array}{l}\text { 153, LUMO+1 } \\
(-2.760)\end{array}$ & $\begin{array}{l}\text { C(bpy): } 72 \% \\
\text { N(bpy): } 23 \% \\
\text { Rh: } 4 \%\end{array}$ & \\
\hline $\begin{array}{c}\text { 152, LUMO } \\
(-3.133)\end{array}$ & $\begin{array}{l}\text { C(bpy): } 72 \% \\
\text { N(bpy): } 23 \% \\
\text { Rh: } 4 \%\end{array}$ & \\
\hline
\end{tabular}




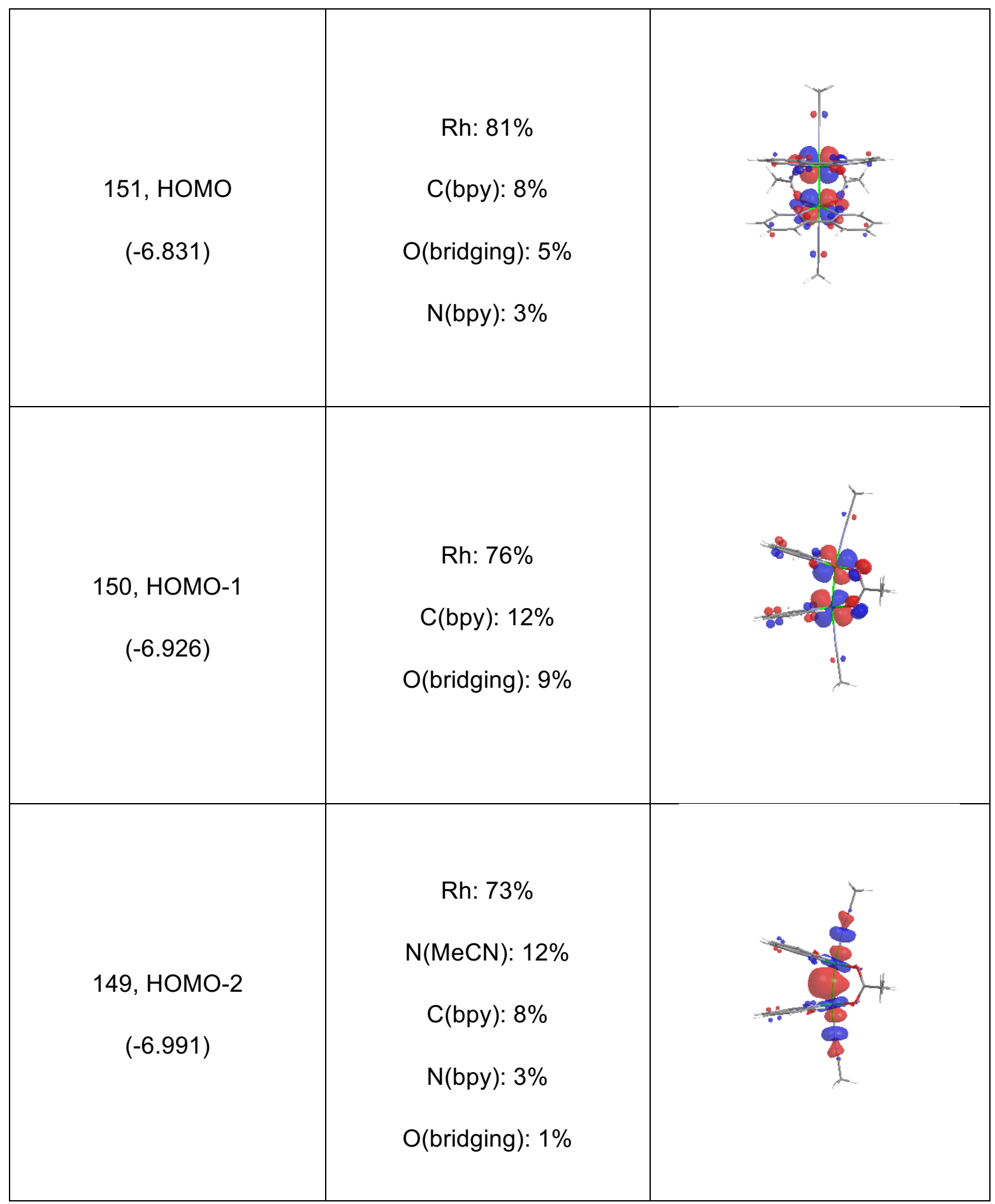




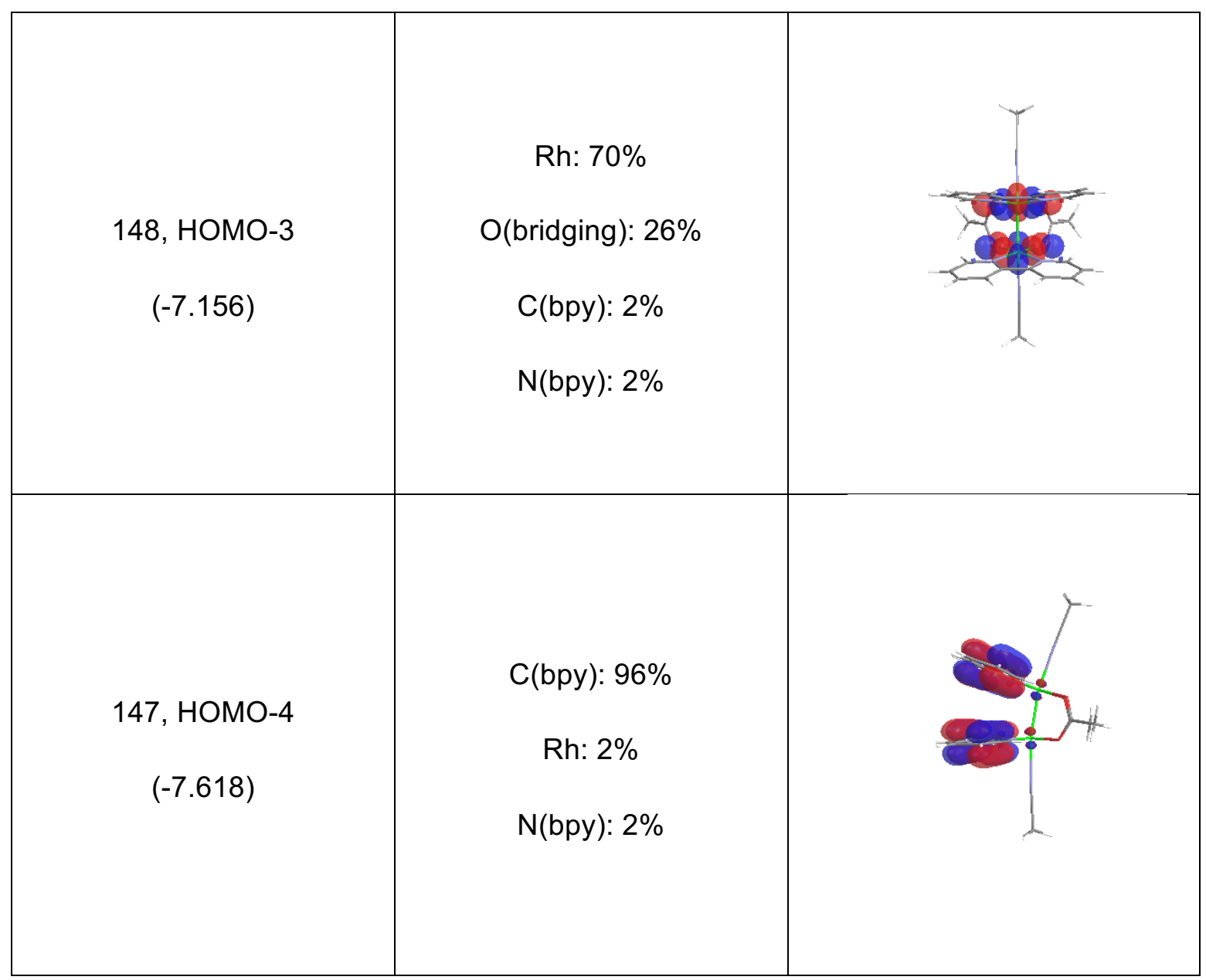



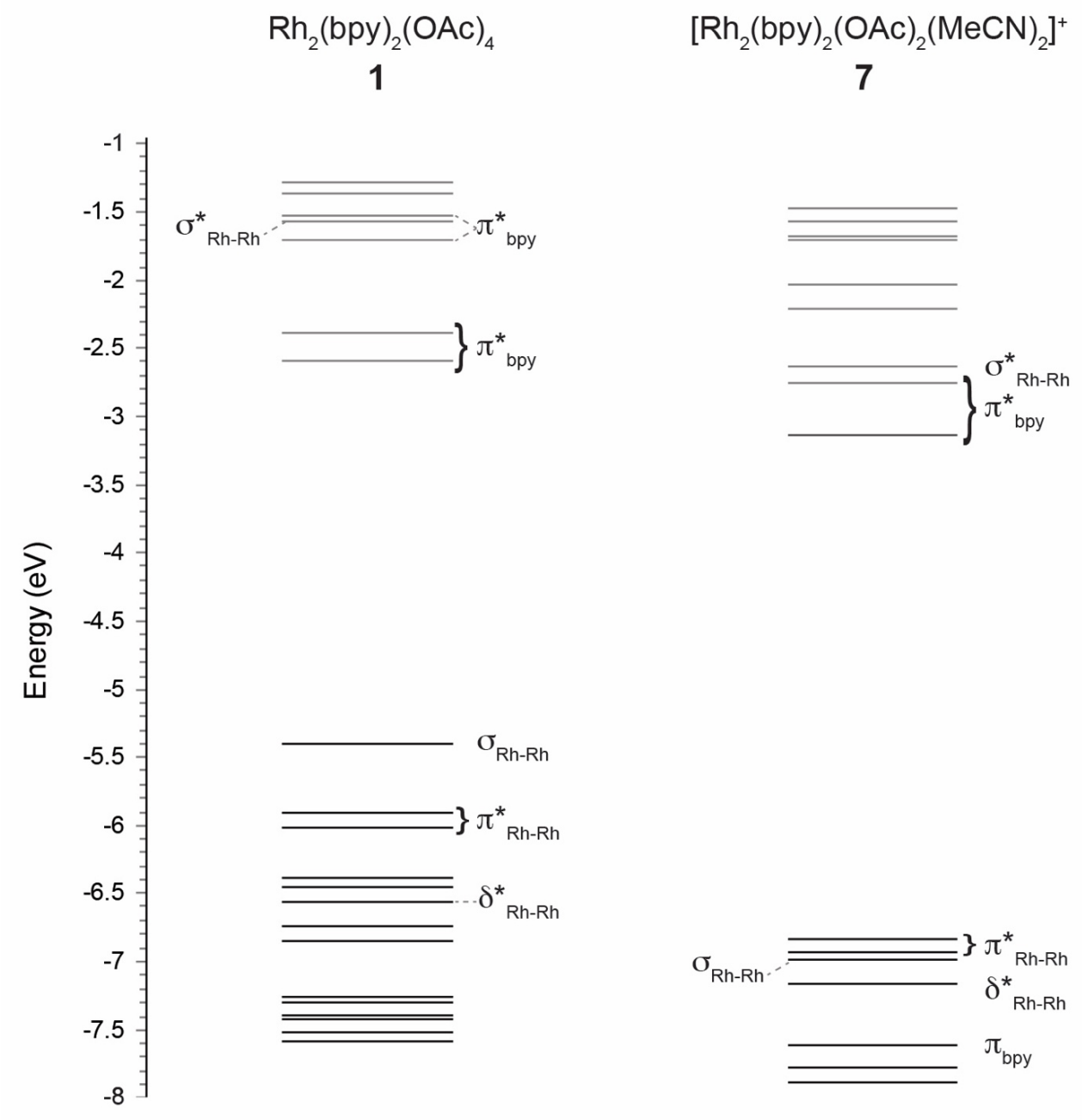

Figure S4. Calculated frontier molecular orbital diagrams for $\mathbf{1}$ and $\mathbf{7}$ (B3PW91/LanL2DZ), showing differences in both the energies and relative ordering of the $\mathrm{Rh}-\mathrm{Rh}$ bonding orbitals. 


\section{Fluorescence Spectroscopy Data}

Emission spectra were recorded at room temperature on an ISS PC1 spectrofluorimeter, with $440 \mathrm{~nm}$ excitation. Emission spectra of 1 recorded in $\mathrm{MeOH}, \mathrm{MeCN}$, and DMF consistently displayed an emission maximum of $468-469 \mathrm{~nm}$.

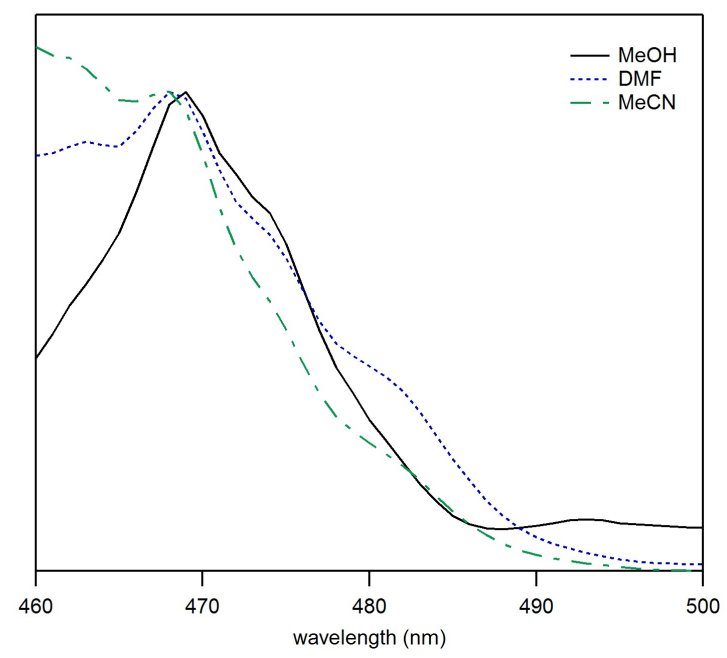

Figure S5. Room temperature emission spectra for complex $1\left(\lambda_{\mathrm{ex}}=440 \mathrm{~nm}\right)$.

\section{Electrochemical Data}

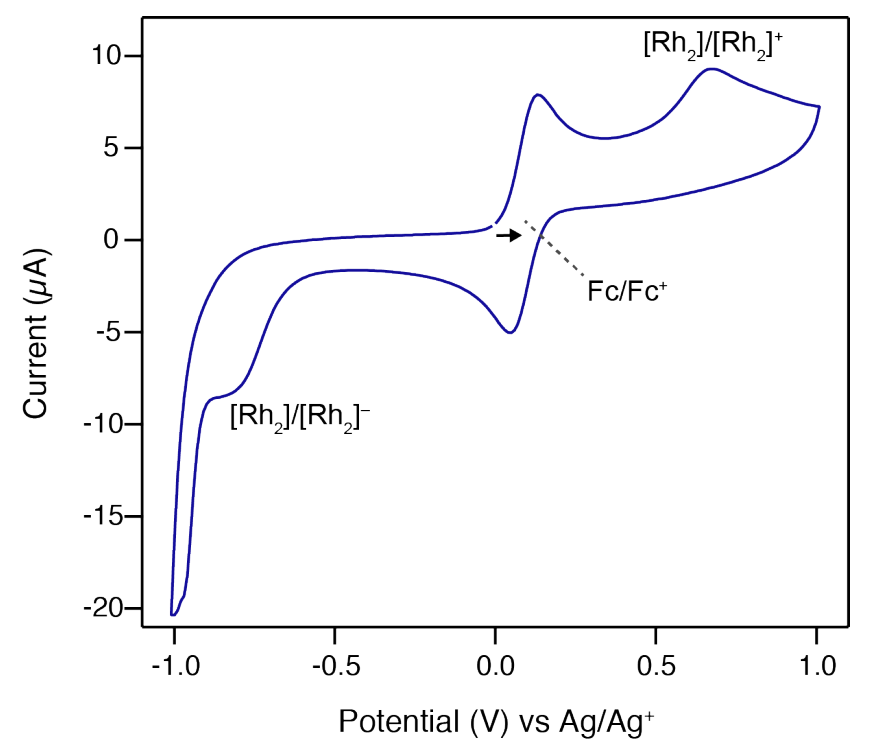

Figure S6. Cyclic voltammogram for complex 1, with ferrocene added as an internal reference (0.1 M TBAPF $6 / \mathrm{MeCN}$; glassy carbon working electrode; Pt wire counter electrode; non-aqueous $\mathrm{Ag} / \mathrm{Ag}^{+}$reference electrode). The starting point and direction of the scan is indicated by an arrow. 


\section{NMR Spectroscopy Data}

${ }^{1} \mathrm{H}$ NMR of $1, \mathrm{CD}_{3} \mathrm{OD}, 300 \mathrm{MHz}, 25^{\circ} \mathrm{C}$

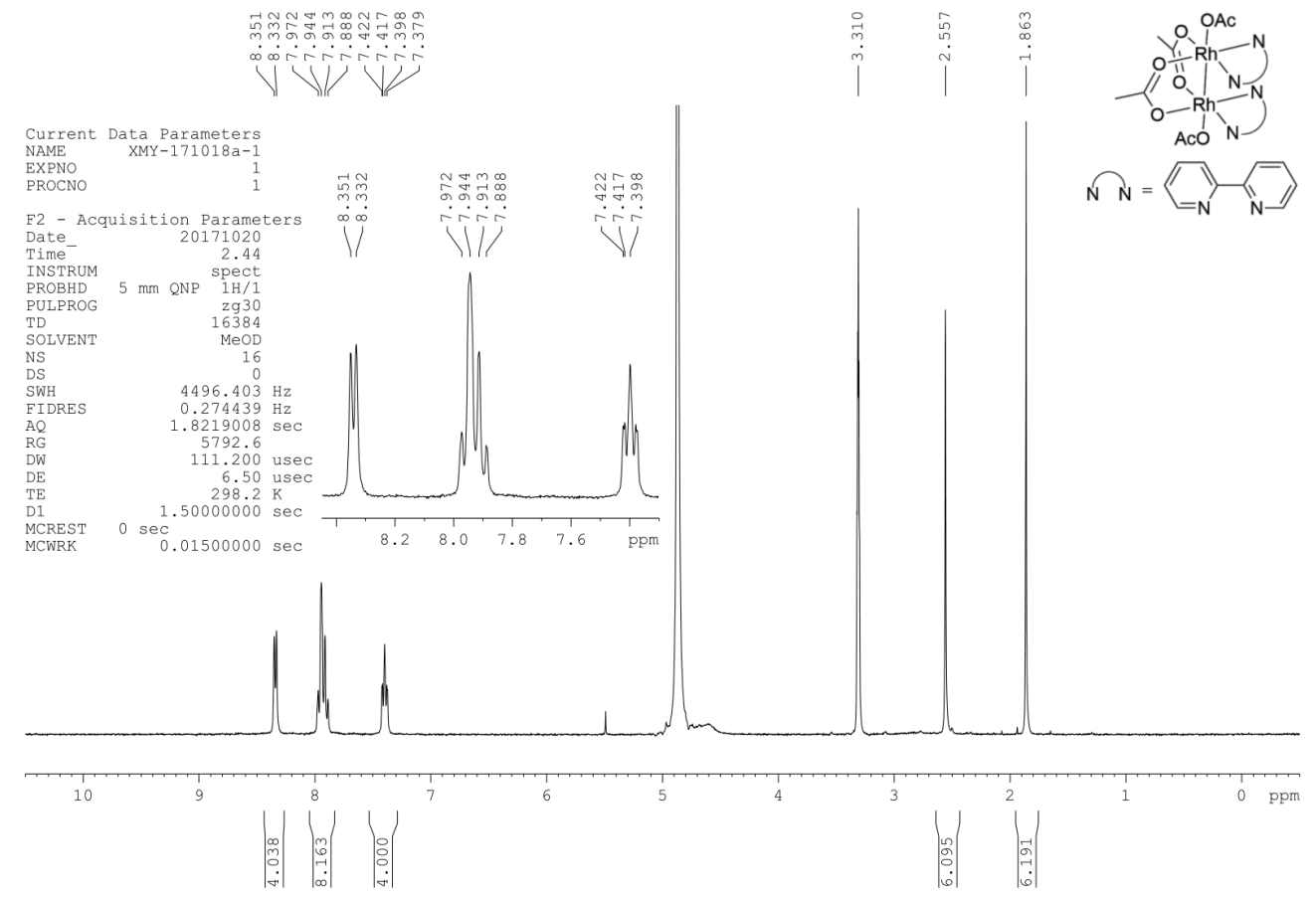

${ }^{13} \mathrm{C}$ NMR of $1, \mathrm{CD}_{3} \mathrm{OD}, 75 \mathrm{MHz}, 24{ }^{\circ} \mathrm{C}$

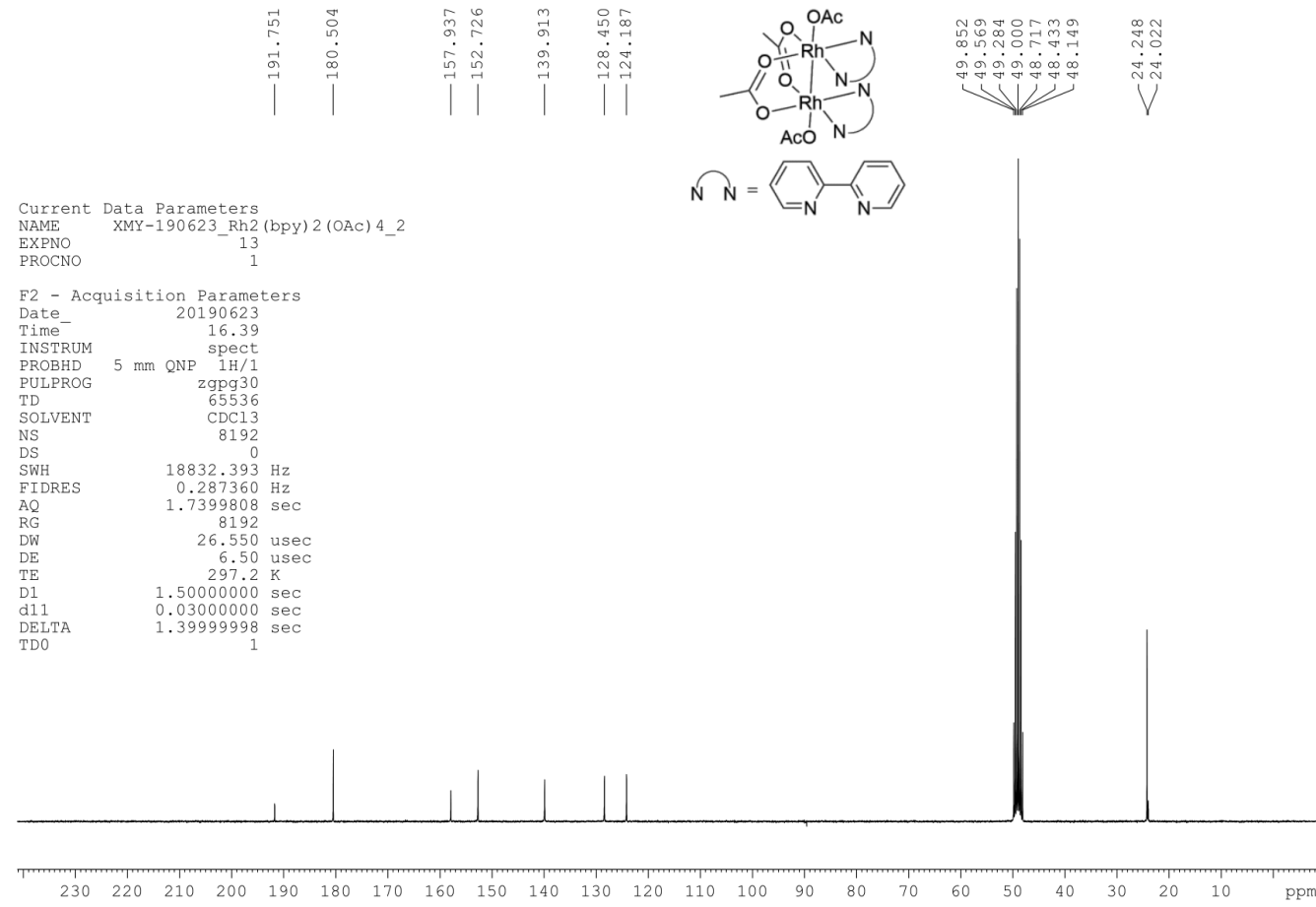


${ }^{1} \mathrm{H}$ NMR of 2, $\mathrm{CD}_{3} \mathrm{OD}, 300 \mathrm{MHz}, 24{ }^{\circ} \mathrm{C}$

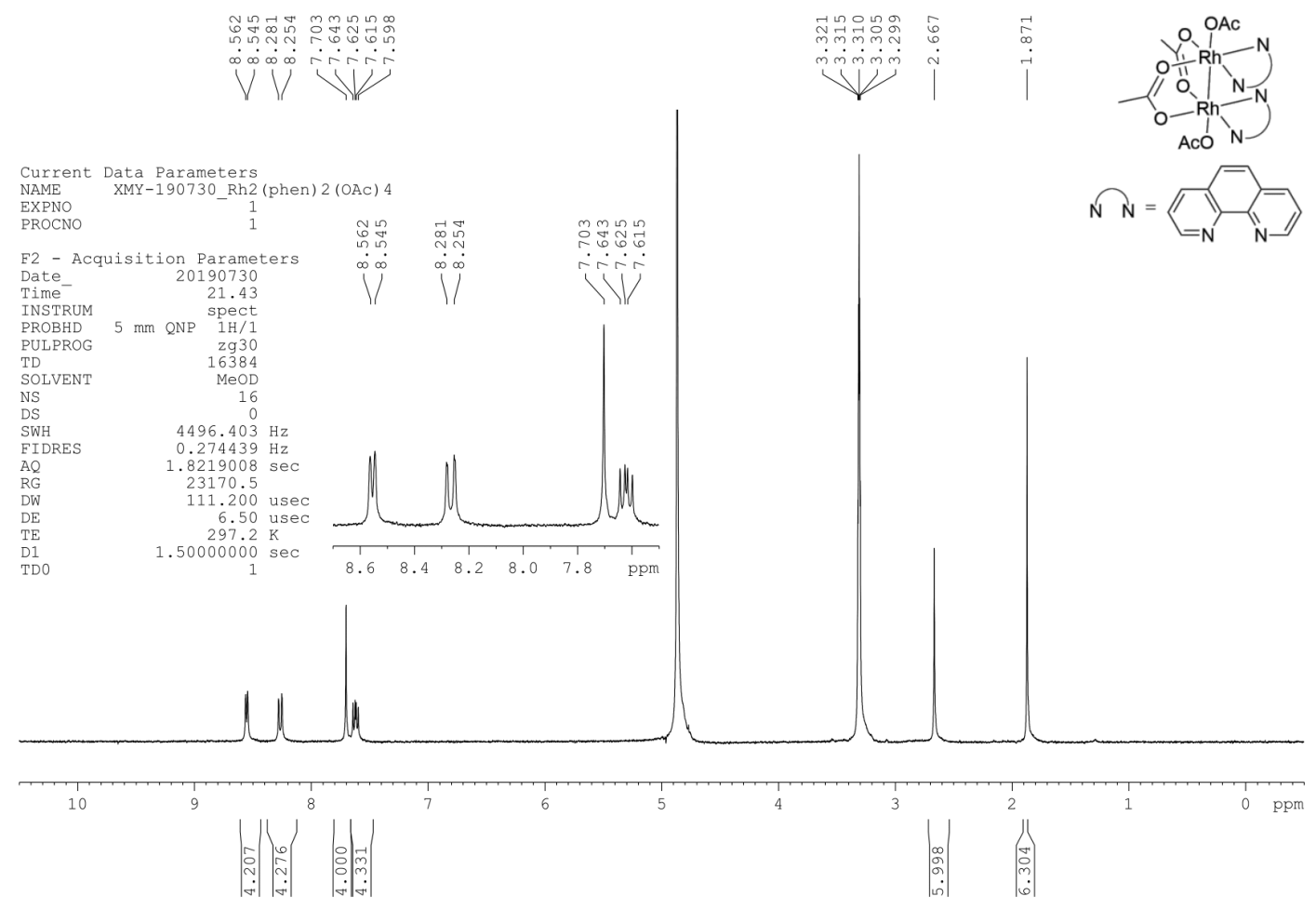

${ }^{13} \mathrm{C}$ NMR of $2, \mathrm{CD}_{3} \mathrm{OD}, 75 \mathrm{MHz}, 24^{\circ} \mathrm{C}$

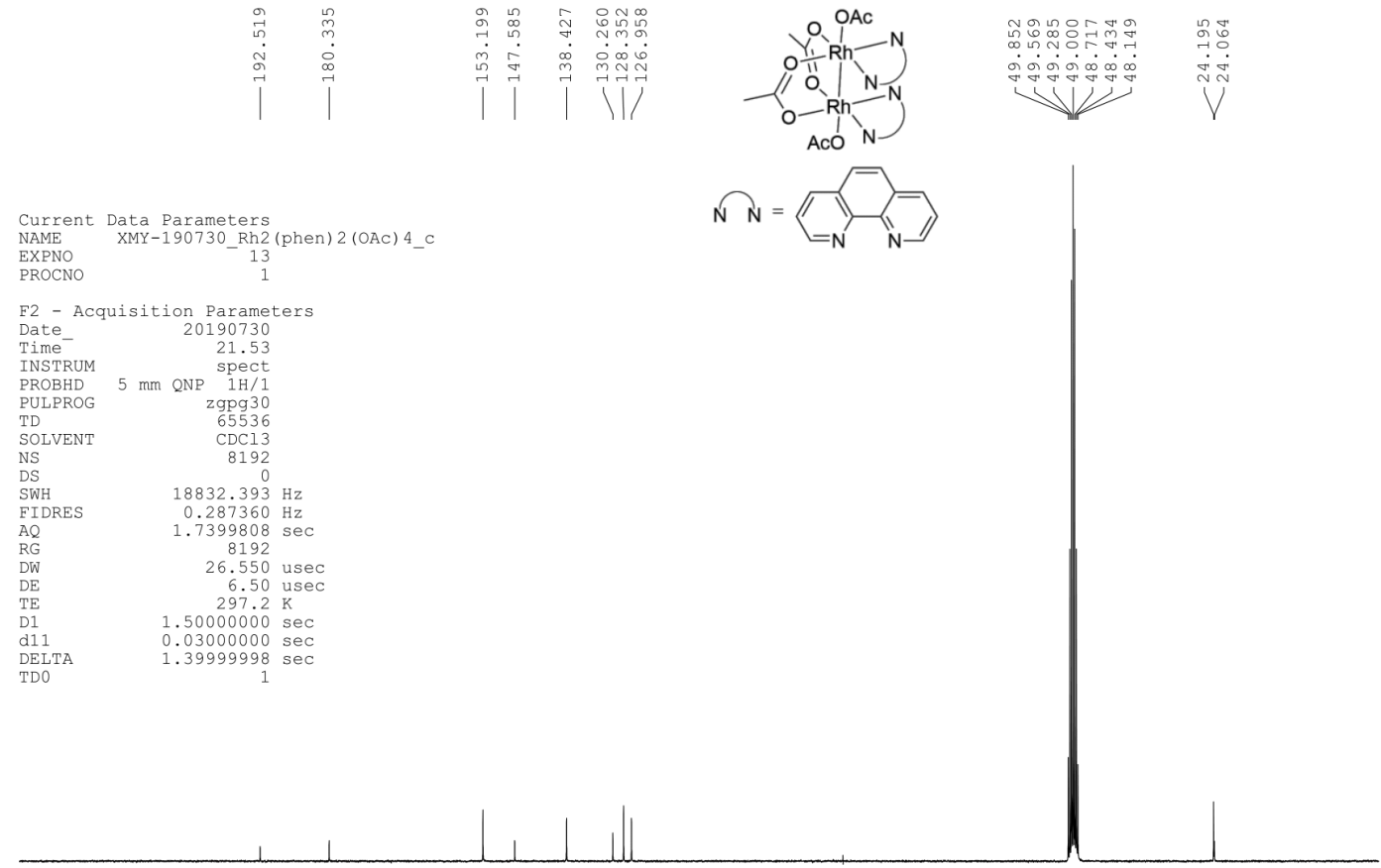

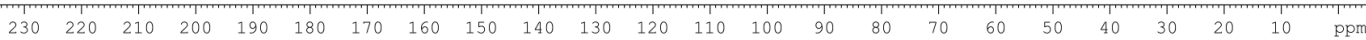


${ }^{1} \mathrm{H}$ NMR of 3, $\mathrm{CD}_{3} \mathrm{OD}: \mathrm{D}_{2} \mathrm{O}=2: 1,300 \mathrm{MHz}, 2{ }^{\circ} \mathrm{C}$

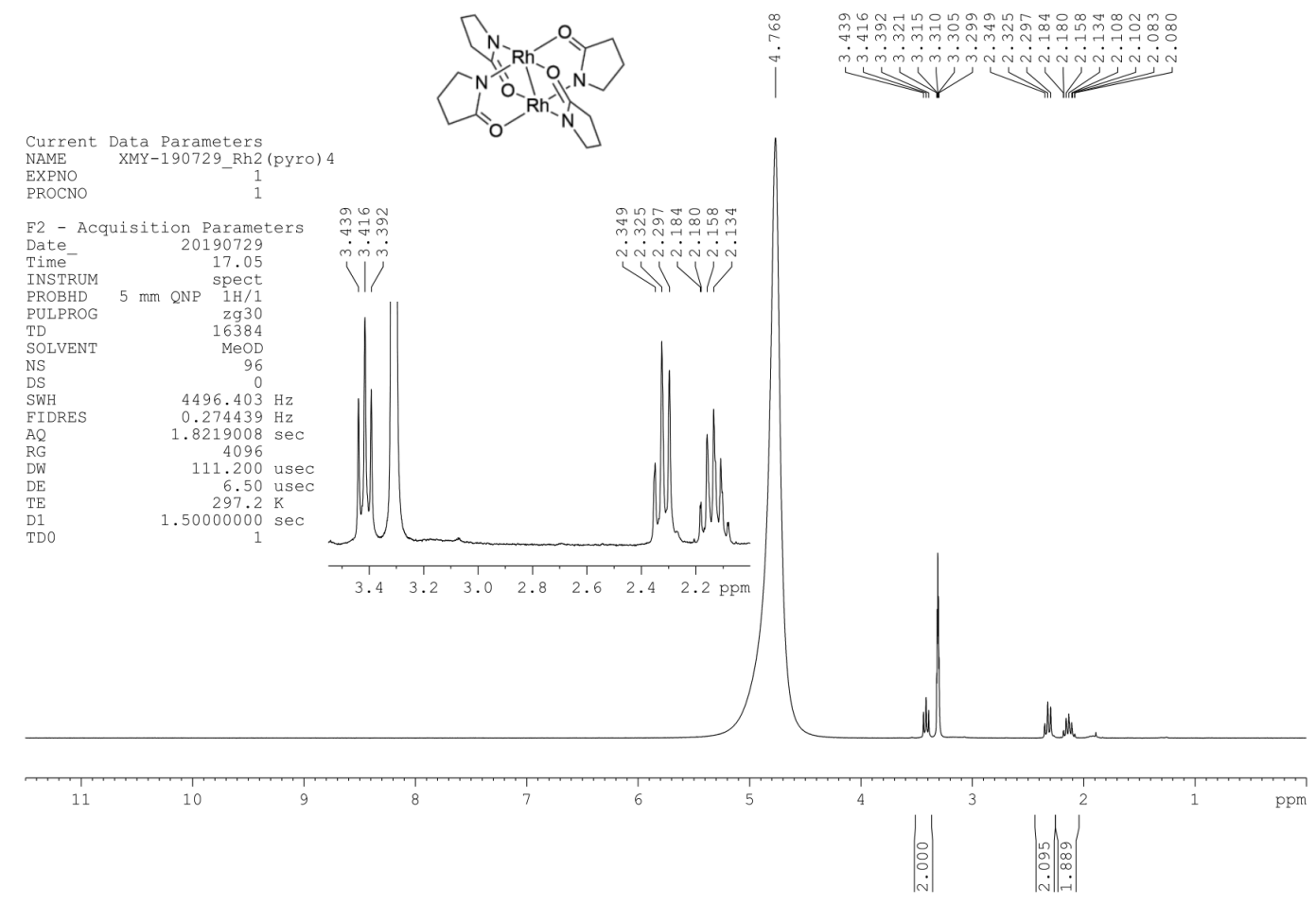

${ }^{1} \mathrm{H}$ NMR of $6 \mathrm{a}, \mathrm{CDCl}_{3}, 400 \mathrm{MHz}, 21^{\circ} \mathrm{C}$

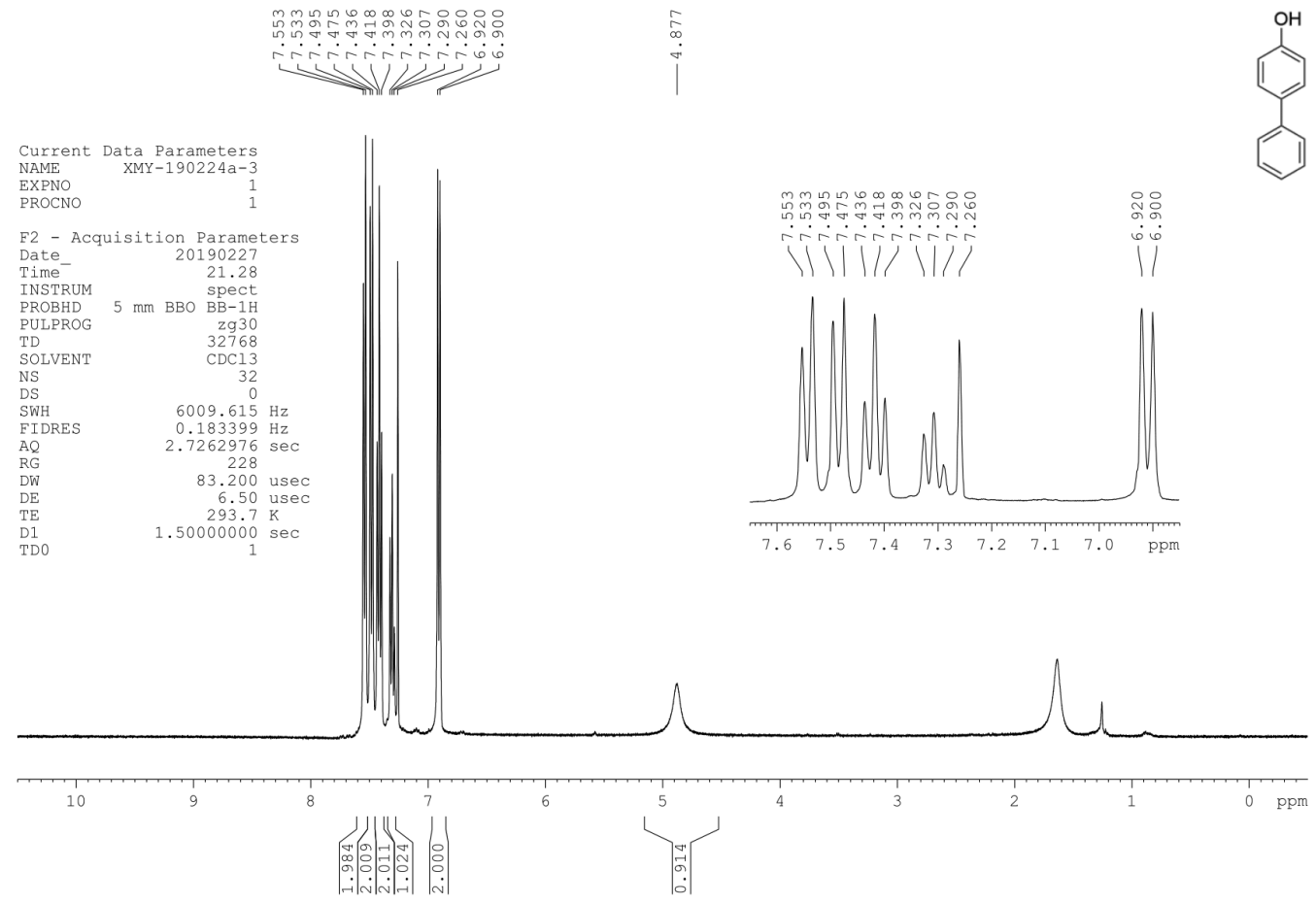


${ }^{13} \mathrm{C}$ NMR of $6 \mathrm{a}, \mathrm{CDCl}_{3}, 100 \mathrm{MHz}, 22{ }^{\circ} \mathrm{C}$
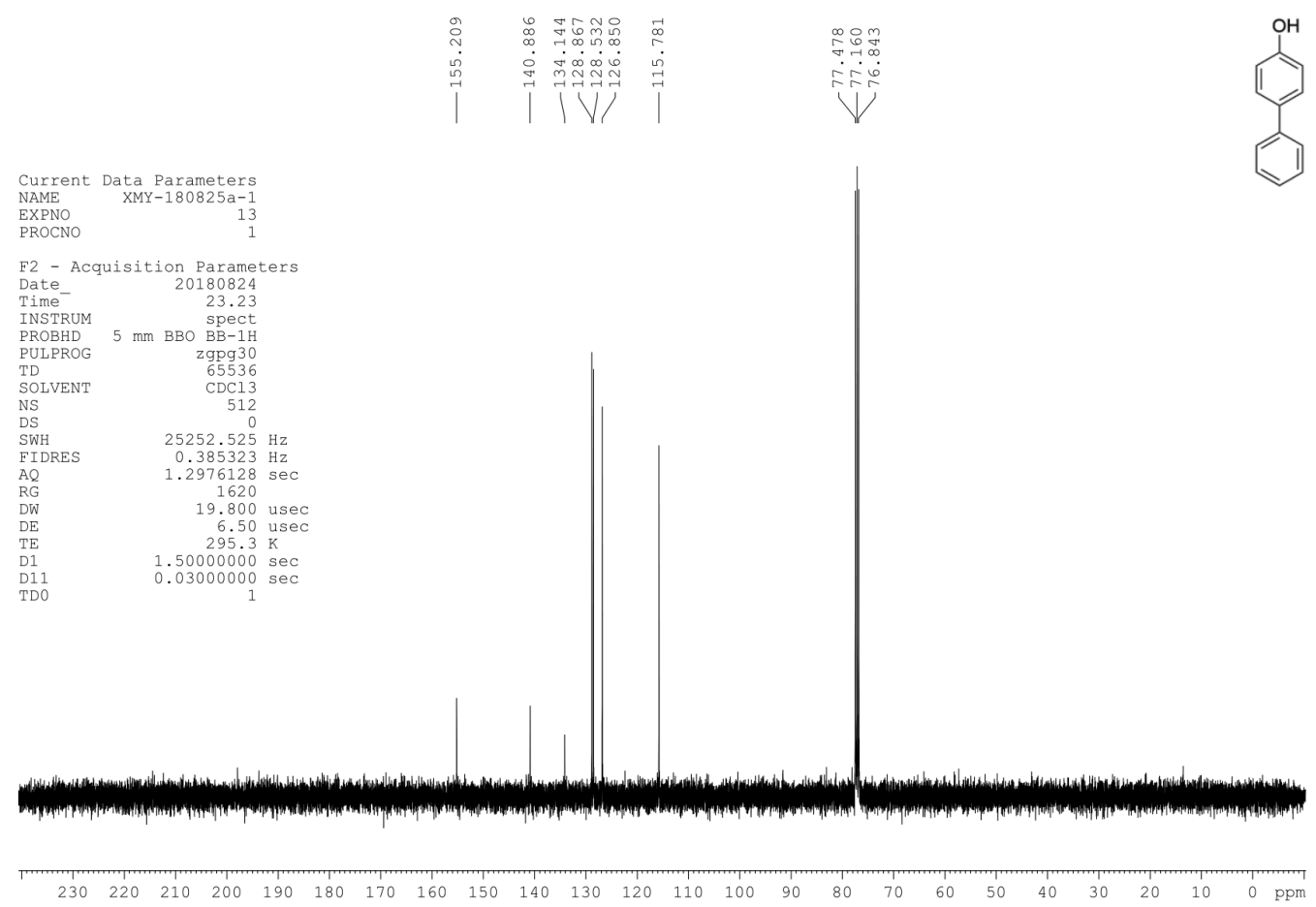

${ }^{1} \mathrm{H}$ NMR of $6 \mathrm{~b}, \mathrm{CDCl}_{3}, 400 \mathrm{MHz}, 21^{\circ} \mathrm{C}$

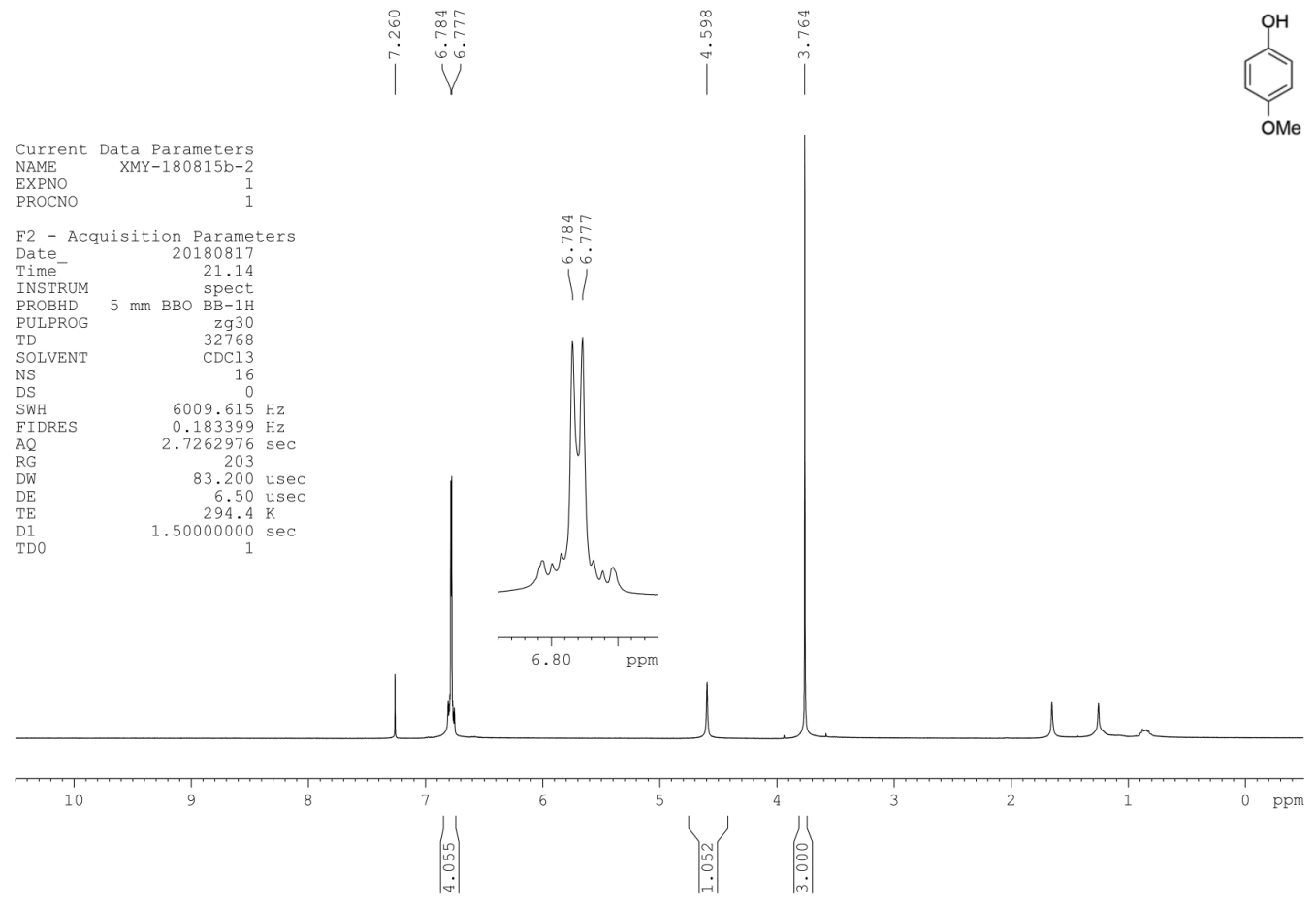


${ }^{13} \mathrm{C}$ NMR of $6 \mathrm{~b}, \mathrm{CDCl}_{3}, 100 \mathrm{MHz}, 22{ }^{\circ} \mathrm{C}$

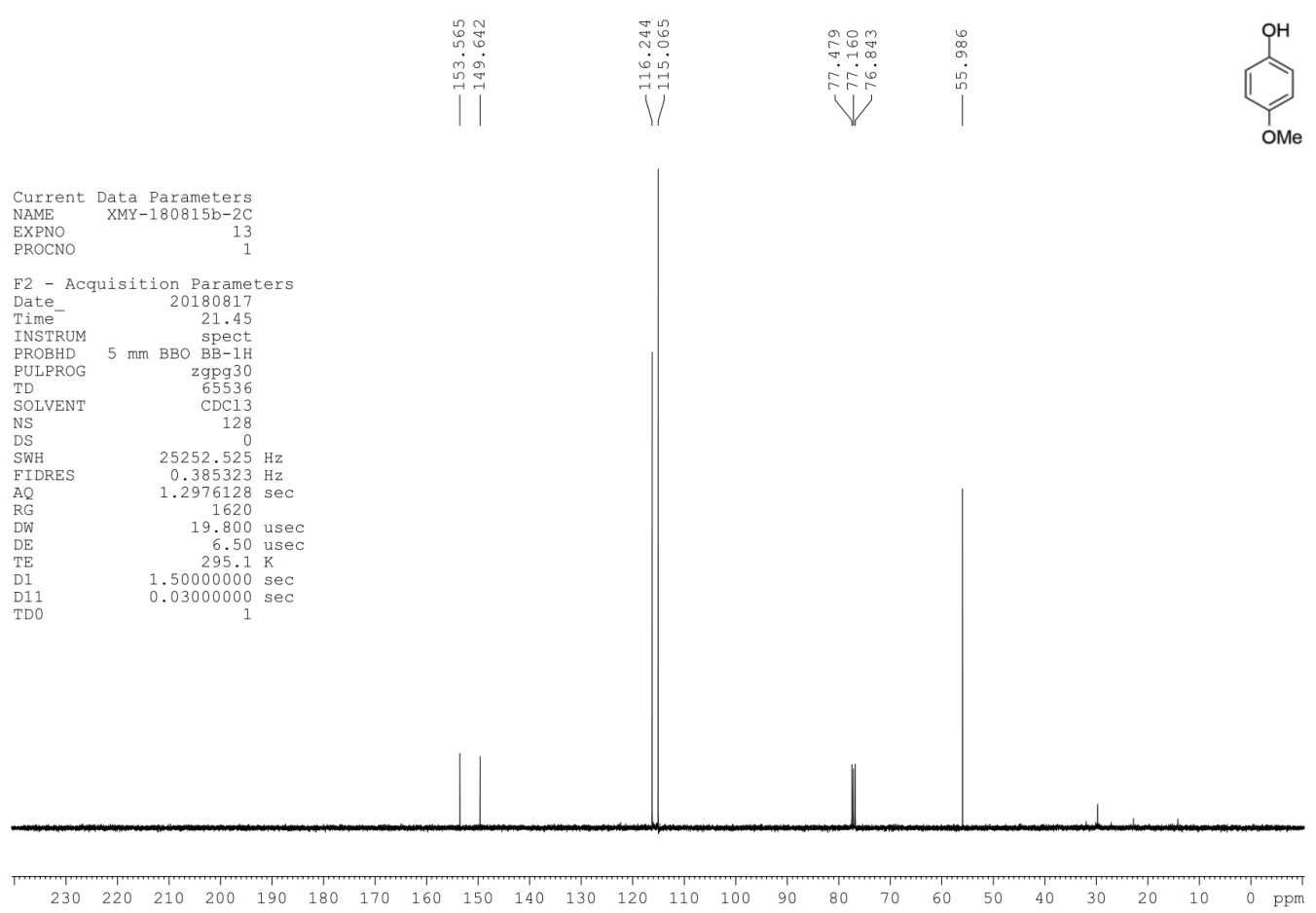

${ }^{1} \mathrm{H}$ NMR of $6 \mathrm{c}, \mathrm{CDCl}_{3}, 400 \mathrm{MHz}, 21^{\circ} \mathrm{C}$

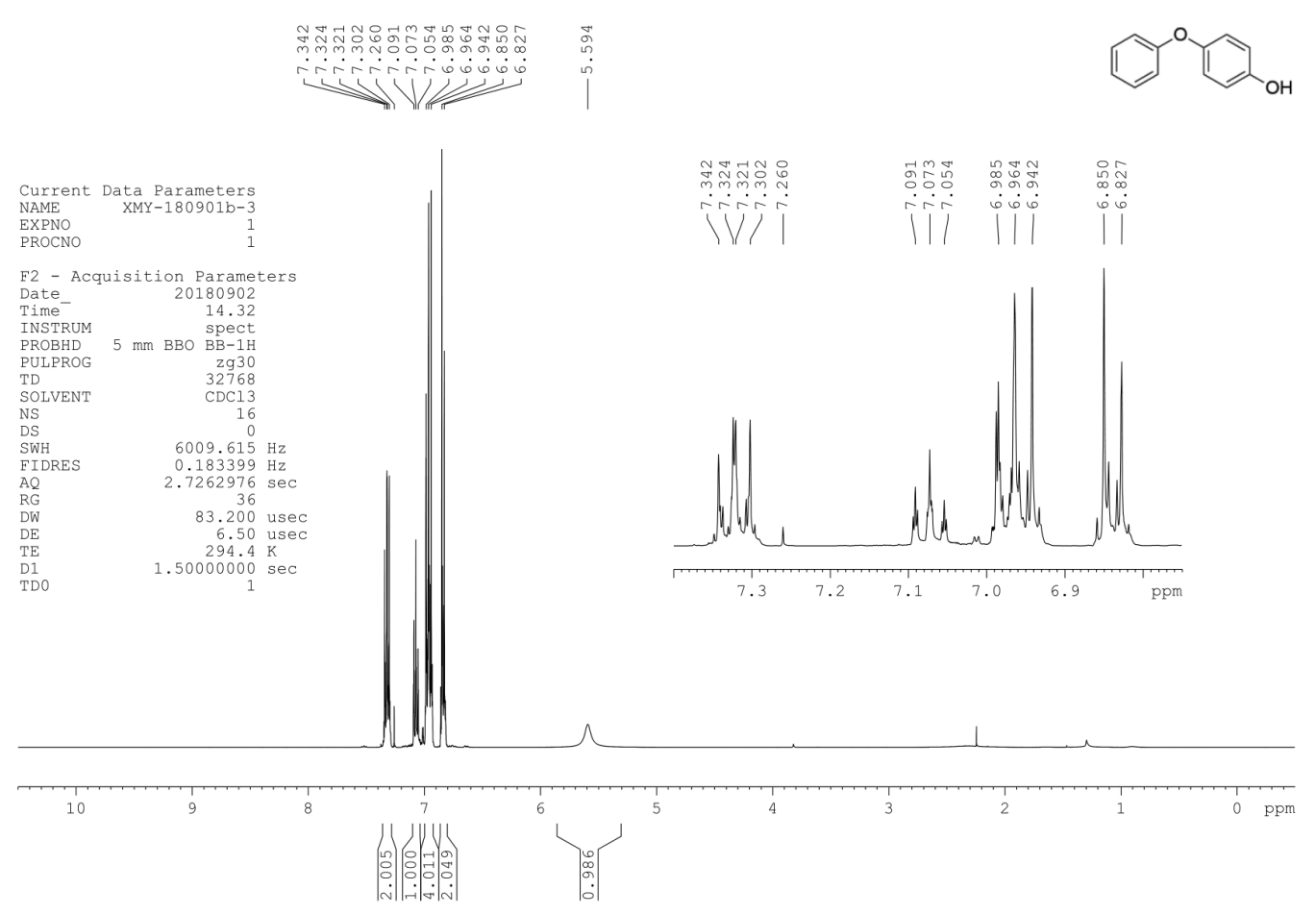


${ }^{13} \mathrm{C}$ NMR of $6 \mathrm{c}, \mathrm{CDCl}_{3}, 100 \mathrm{MHz}, 22{ }^{\circ} \mathrm{C}$

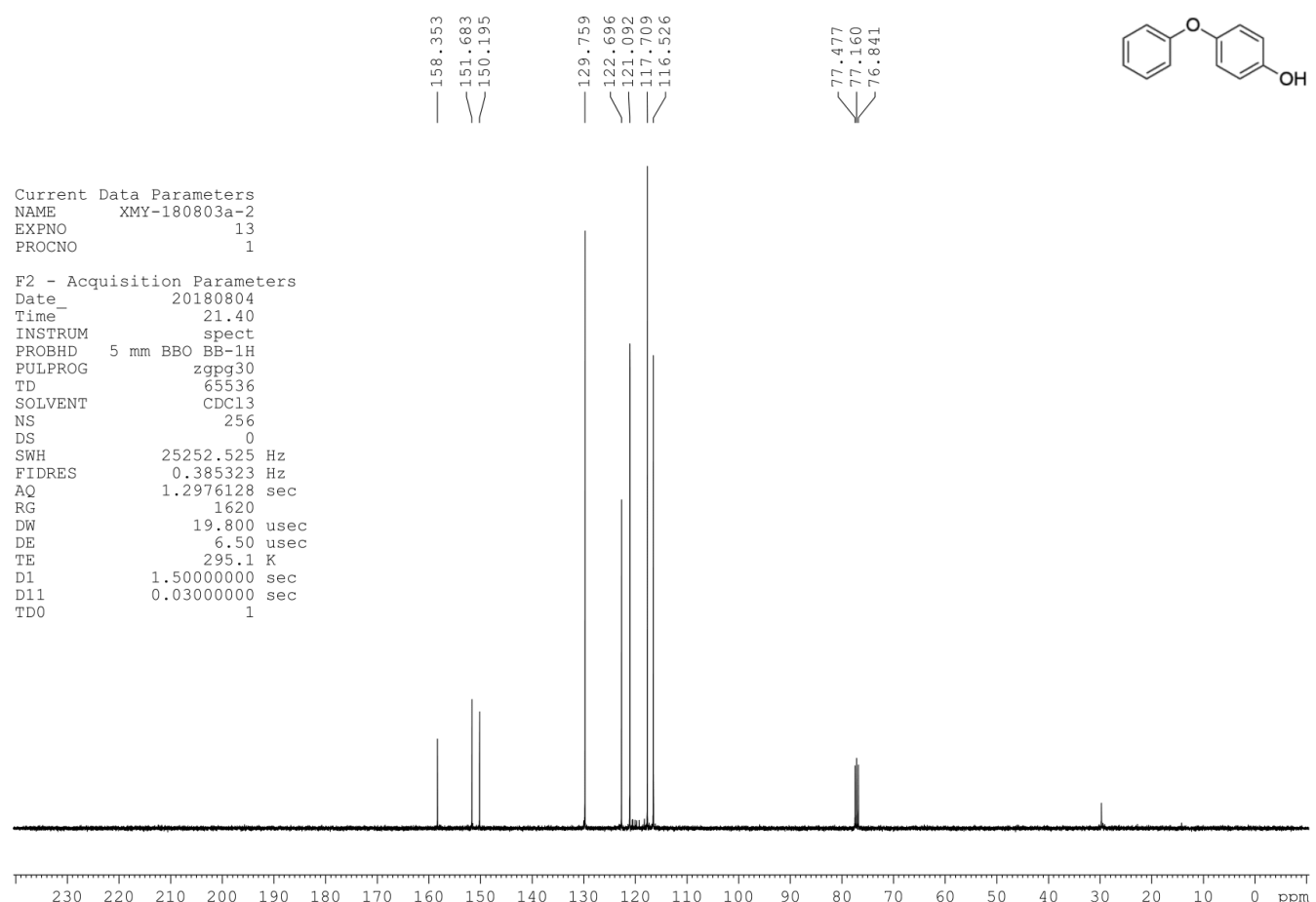

${ }^{1} \mathrm{H}$ NMR of $6 \mathrm{~d}, \mathrm{CDCl}_{3}, 400 \mathrm{MHz}, 21^{\circ} \mathrm{C}$

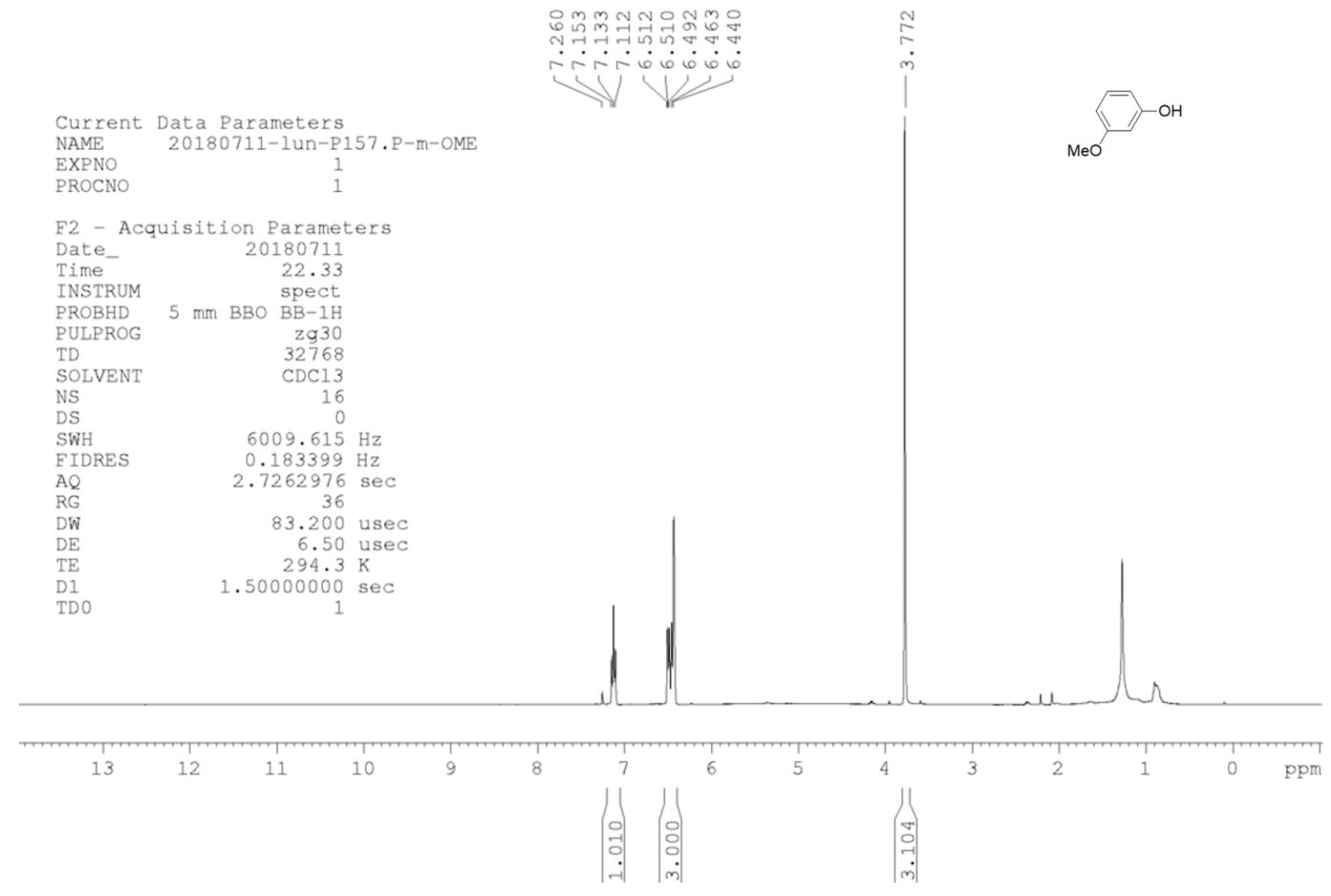


${ }^{13} \mathrm{C}$ NMR of $6 \mathrm{~d}, \mathrm{CDCl}_{3}, 100 \mathrm{MHz}, 22{ }^{\circ} \mathrm{C}$

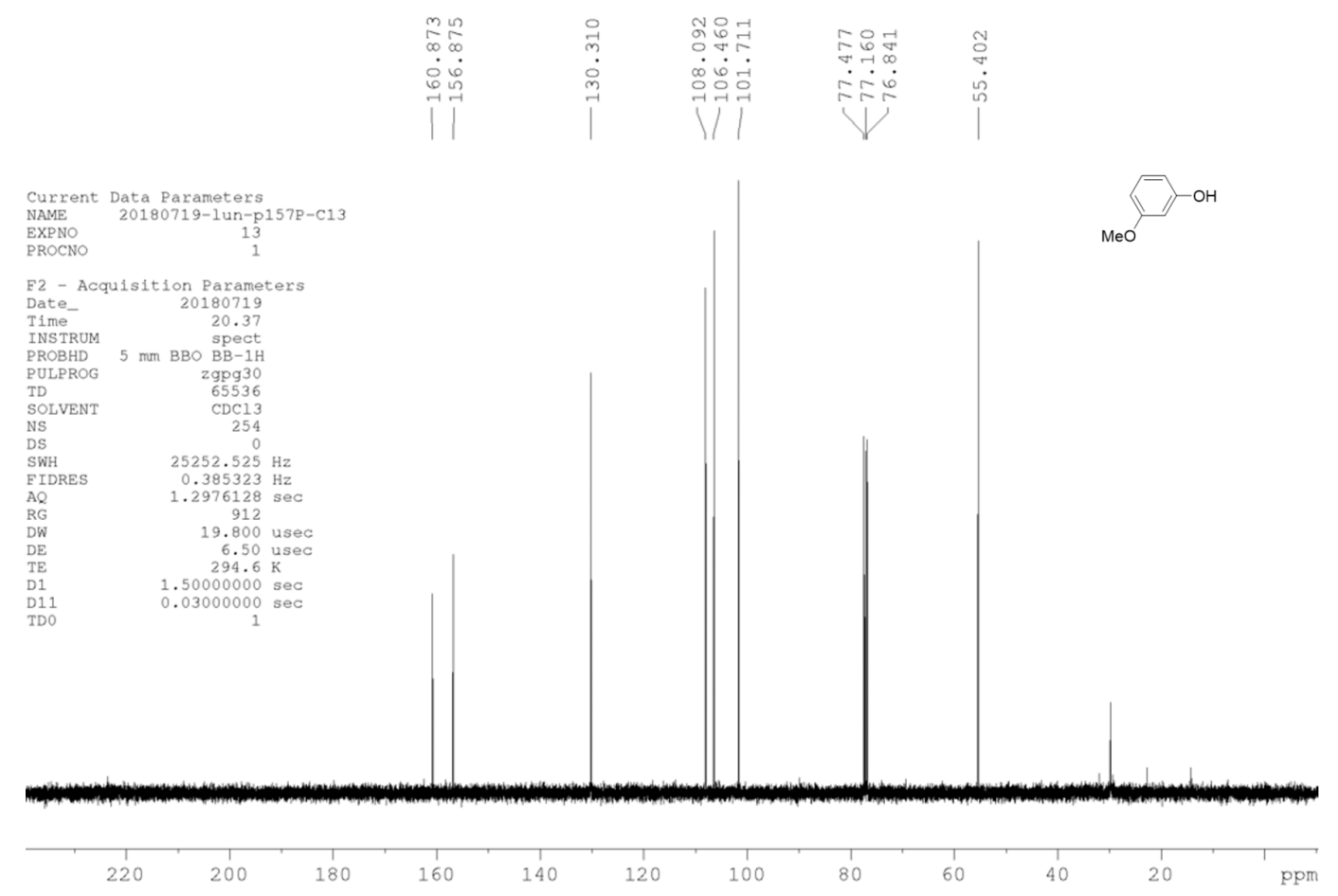

${ }^{1} \mathrm{H}$ NMR of $6 \mathrm{e}, \mathrm{CDCl}_{3}, 400 \mathrm{MHz}, 22^{\circ} \mathrm{C}$
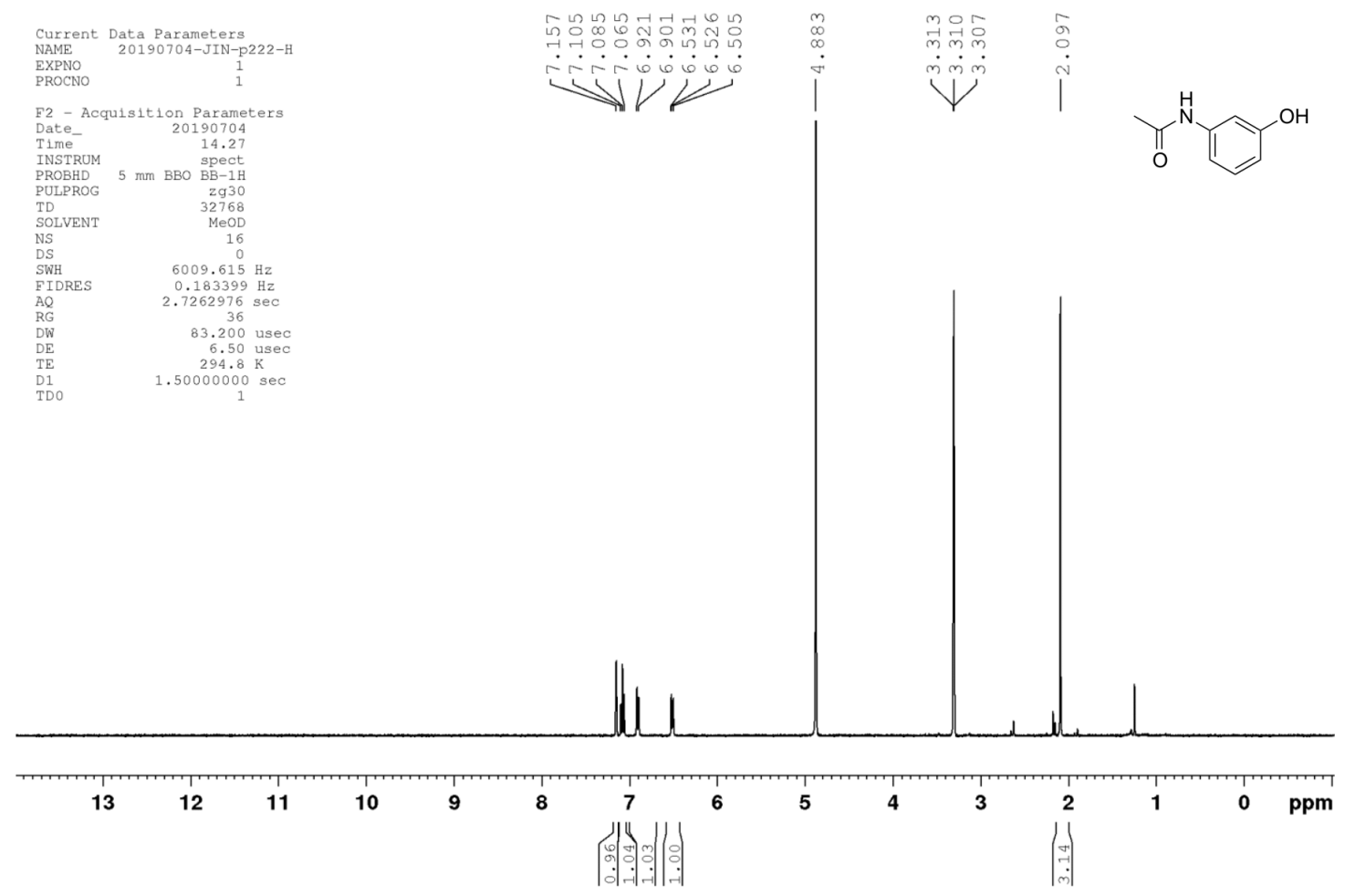
${ }^{13} \mathrm{C}$ NMR of $6 \mathrm{e}, \mathrm{CDCl}_{3}, 100 \mathrm{MHz}, 22{ }^{\circ} \mathrm{C}$
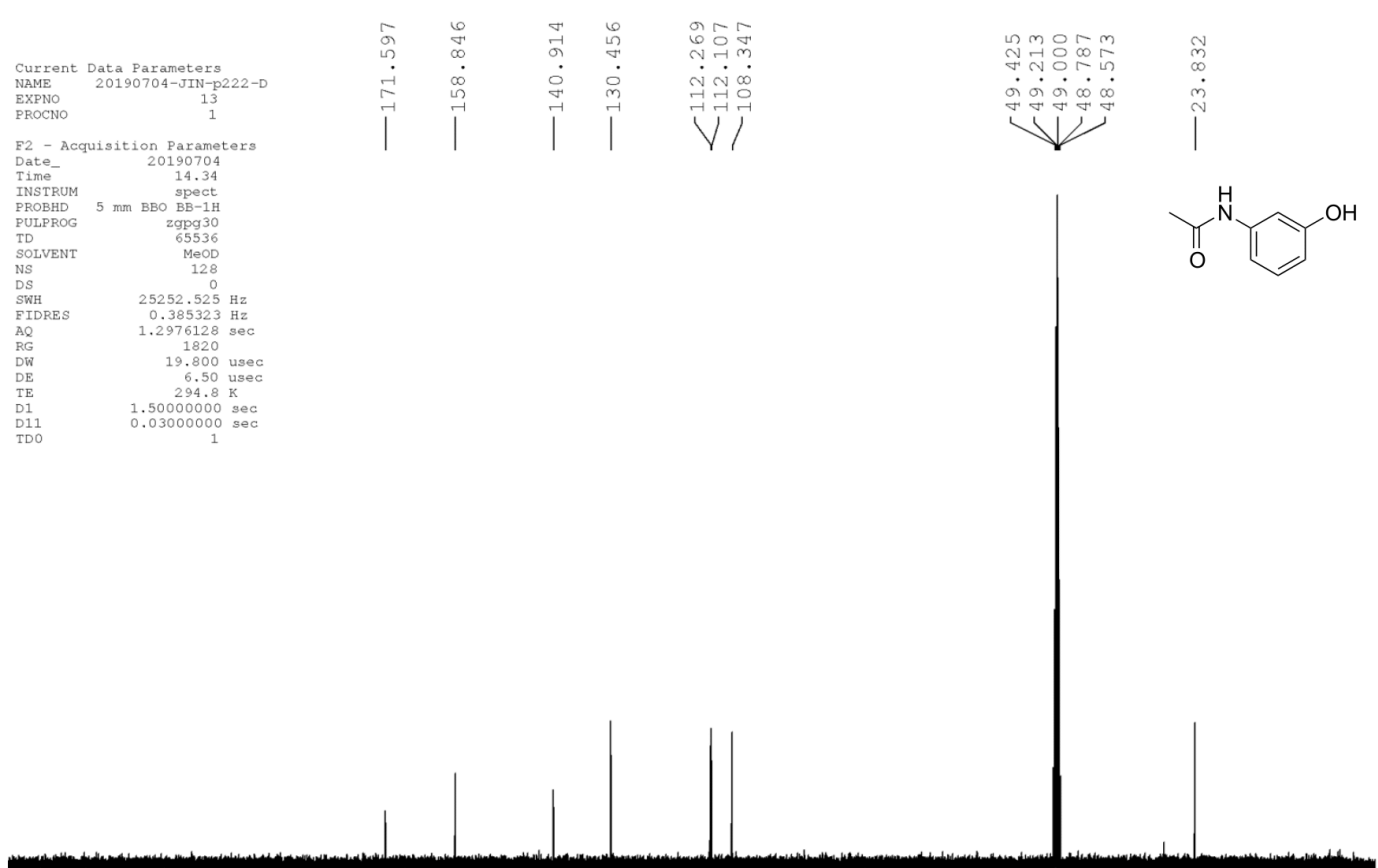

220

${ }^{1} \mathrm{H}$ NMR of $6 \mathrm{f}, \mathrm{CD}_{3} \mathrm{OD}, 400 \mathrm{MHz}, 22^{\circ} \mathrm{C}$
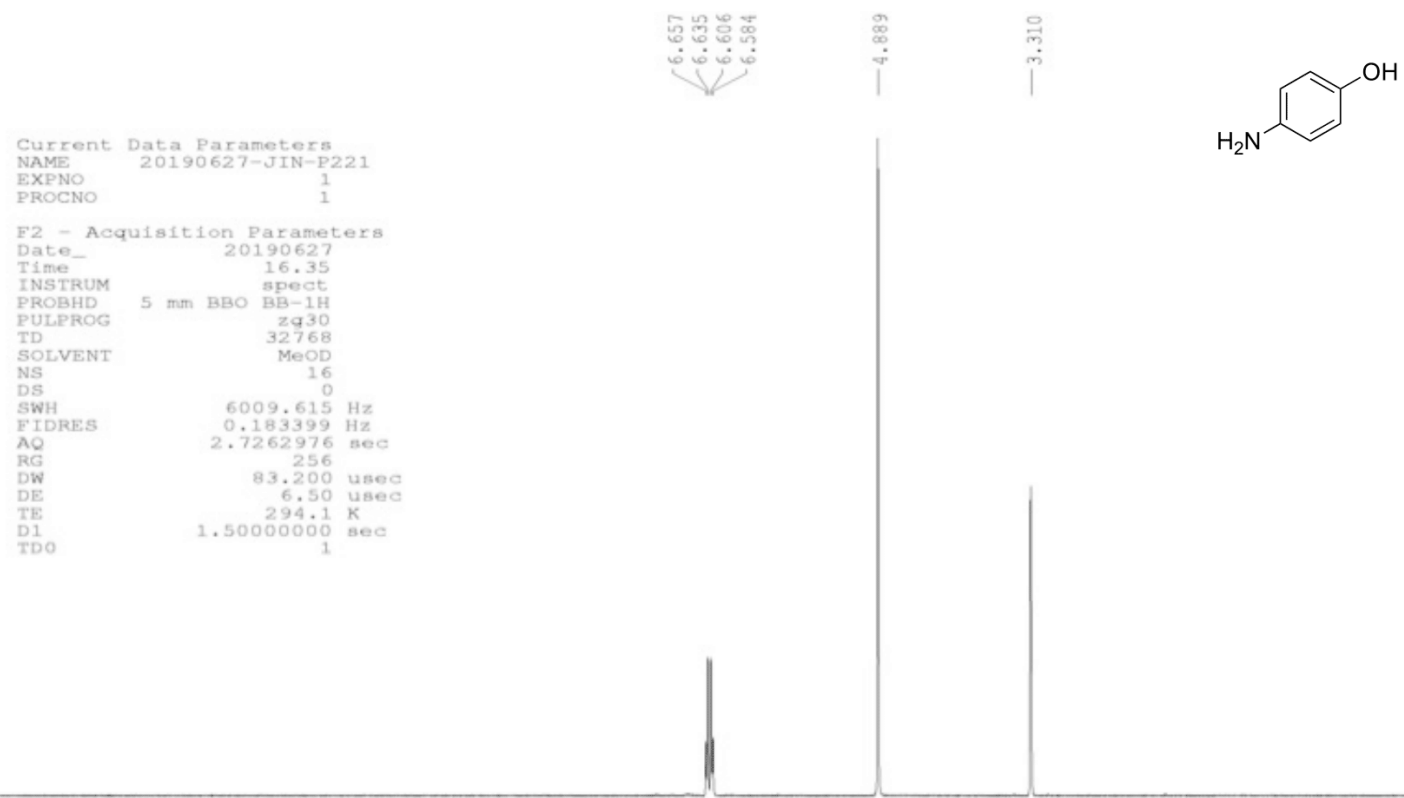

$12 \quad 11 \quad 10$


${ }^{13} \mathrm{C}$ NMR of $6 f, \mathrm{CD}_{3} \mathrm{OD}, 100 \mathrm{MHz}, 22{ }^{\circ} \mathrm{C}$
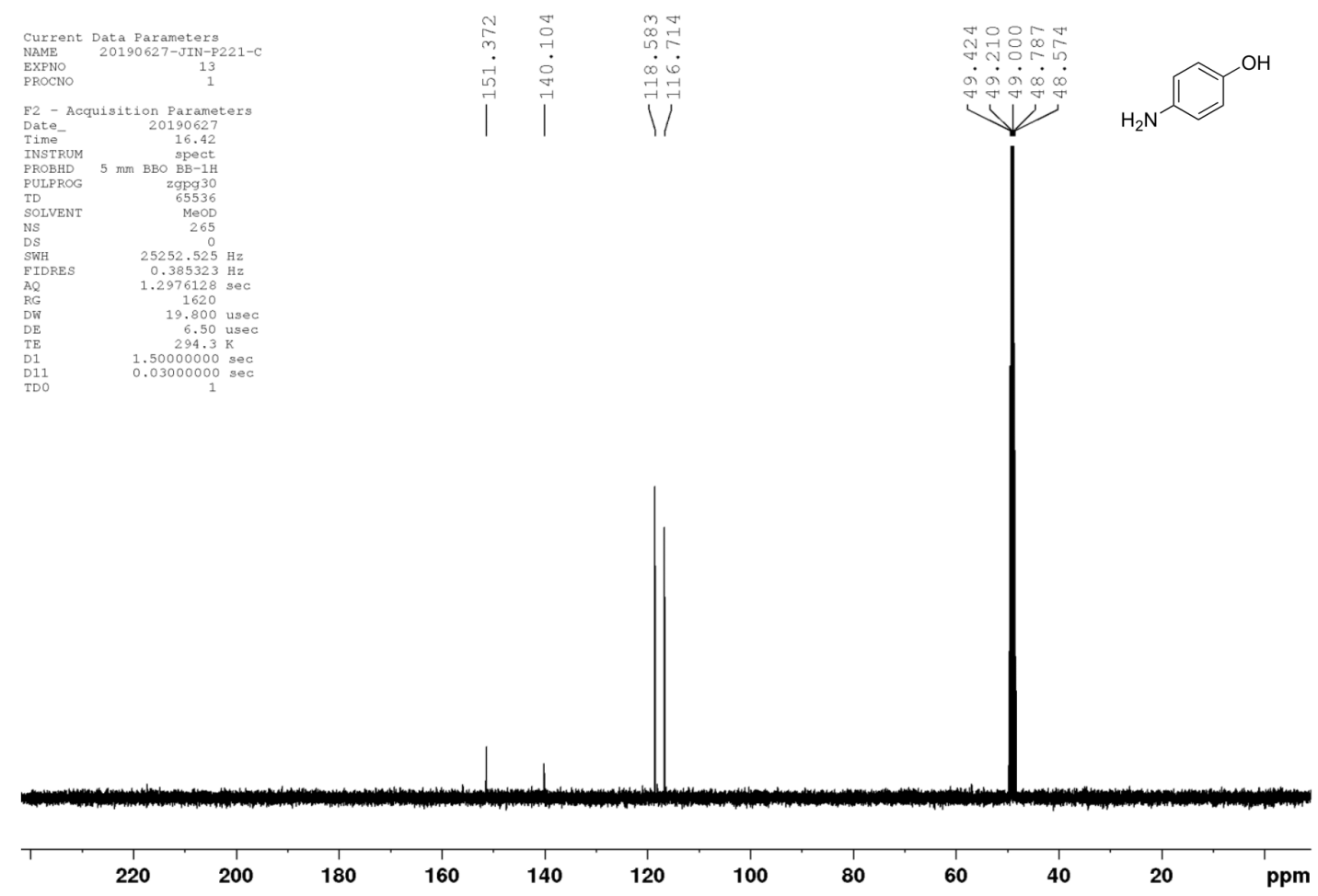

${ }^{1} \mathrm{H}$ NMR of $6 \mathrm{~g}$, DMSO-d $6,400 \mathrm{MHz}, 21^{\circ} \mathrm{C}$

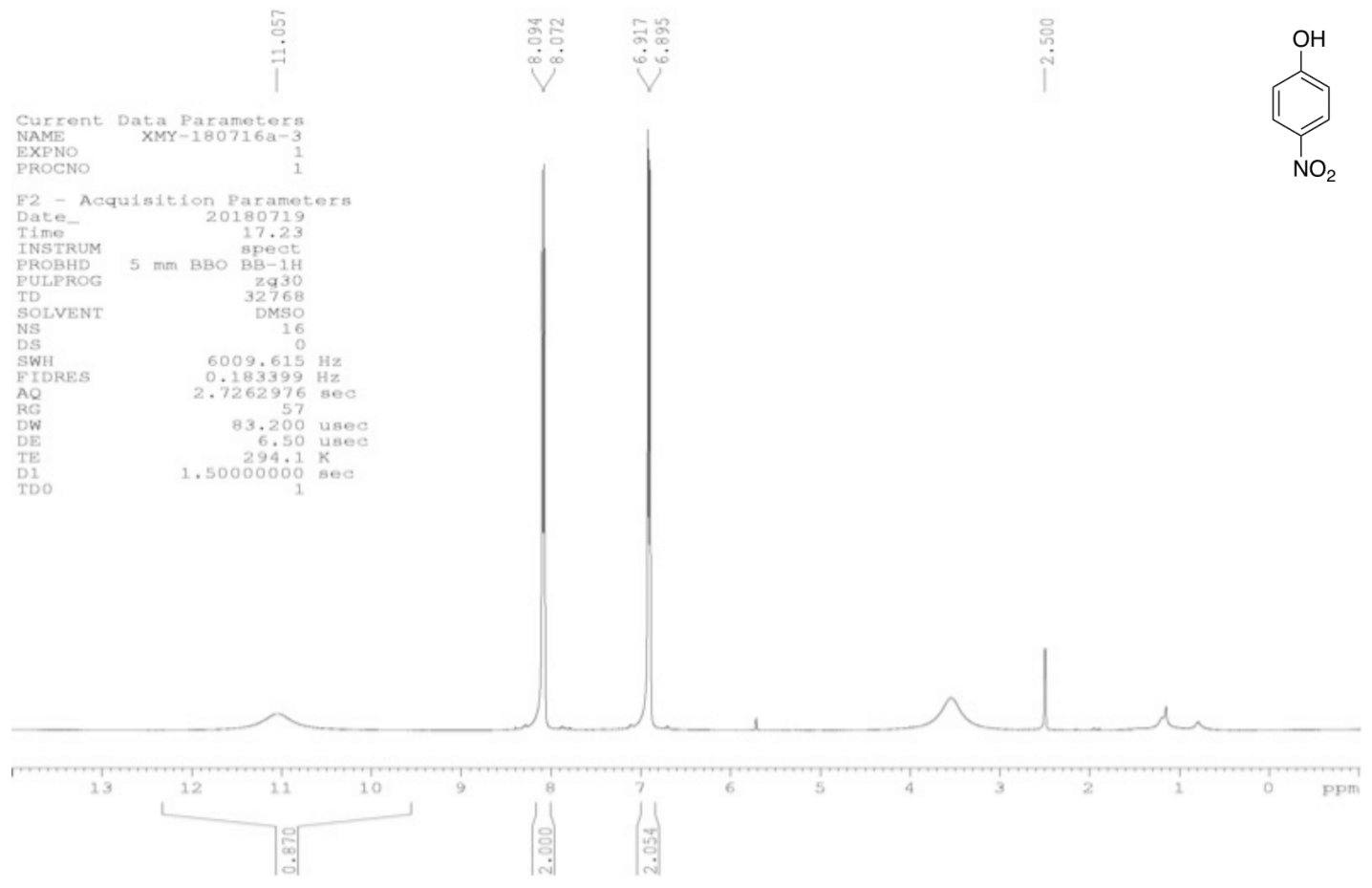


${ }^{13} \mathrm{C}$ NMR of $6 \mathrm{~g}$, DMSO-d $6,100 \mathrm{MHz}, 22{ }^{\circ} \mathrm{C}$
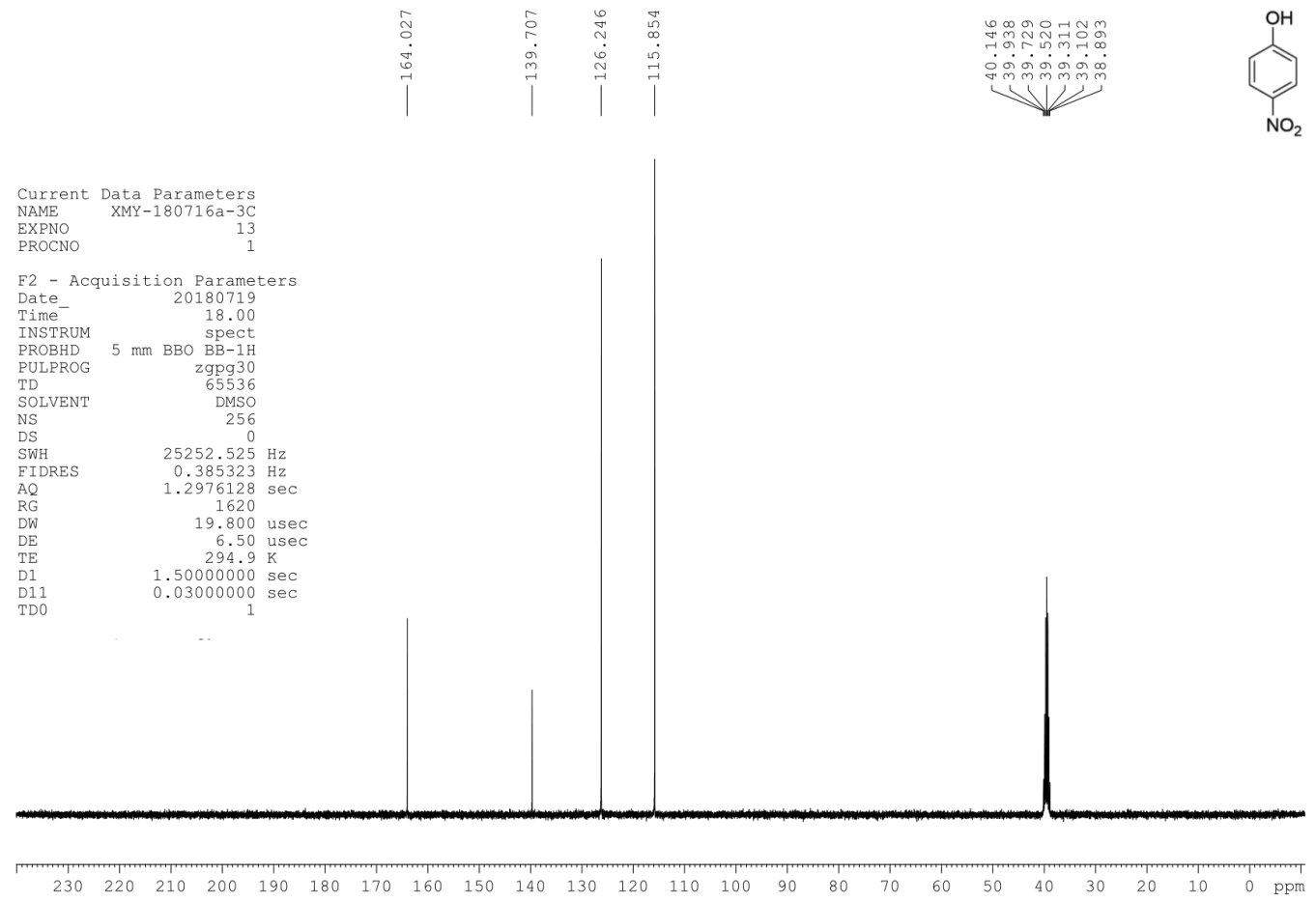

${ }^{1} \mathrm{H}$ NMR of $6 \mathrm{~h}, \mathrm{CDCl}_{3}, 400 \mathrm{MHz}, 22^{\circ} \mathrm{C}$

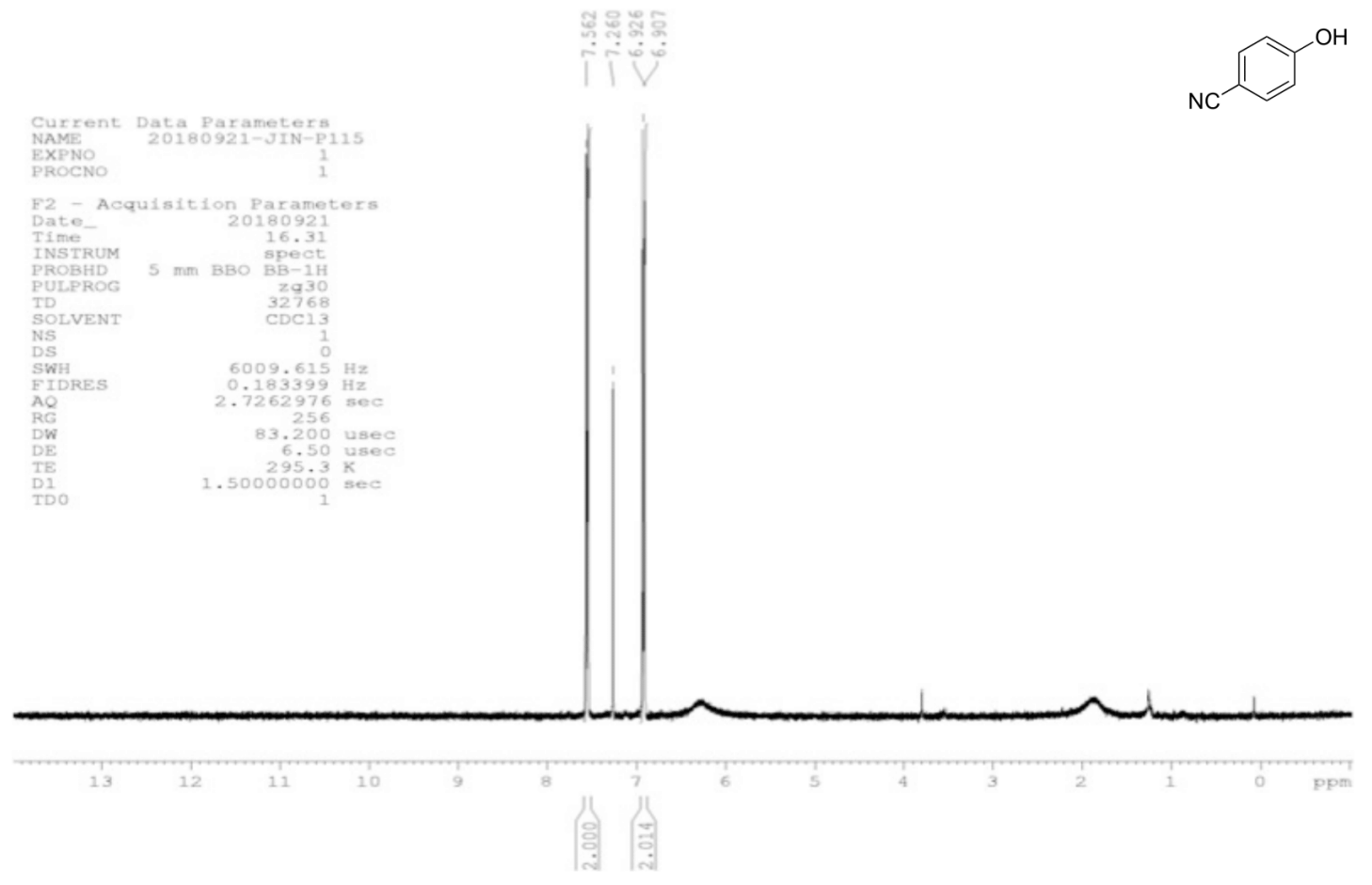


${ }^{13} \mathrm{C}$ NMR of $6 \mathrm{~h}, \mathrm{CDCl}_{3}, 100 \mathrm{MHz}, 22{ }^{\circ} \mathrm{C}$
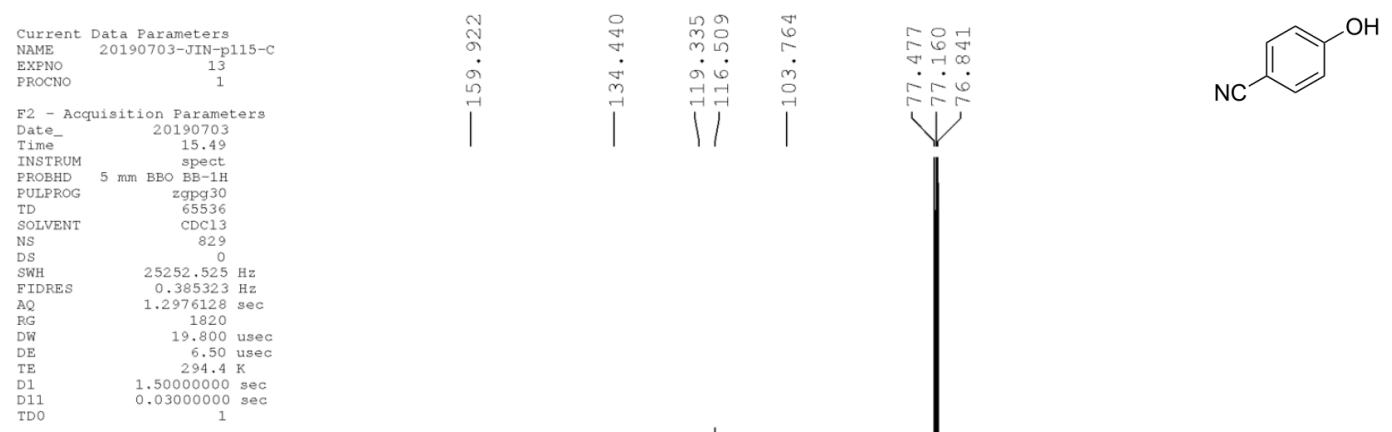

TDO 1

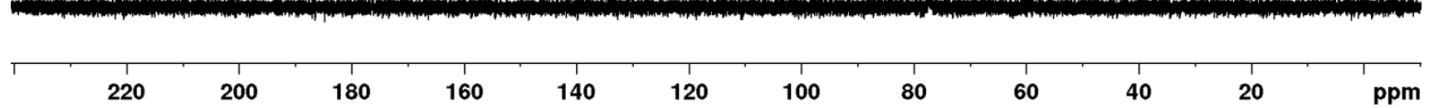

${ }^{1} \mathrm{H}$ NMR of $6 \mathrm{i}, \mathrm{CDCl}_{3}, 400 \mathrm{MHz}, 24{ }^{\circ} \mathrm{C}$
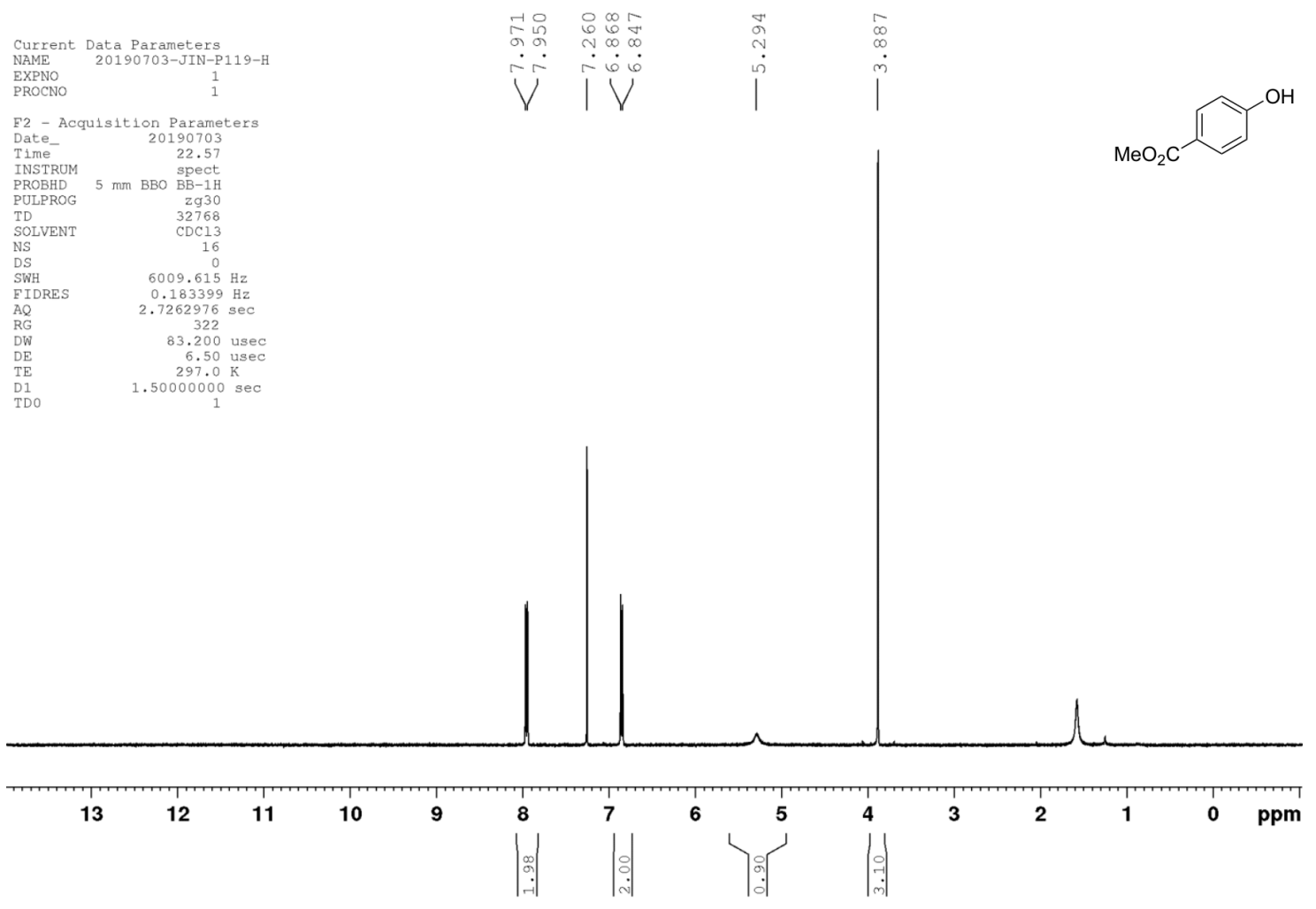
${ }^{13} \mathrm{C}$ NMR of $6 \mathrm{i}, \mathrm{CDCl}_{3}, 100 \mathrm{MHz}, 22^{\circ} \mathrm{C}$

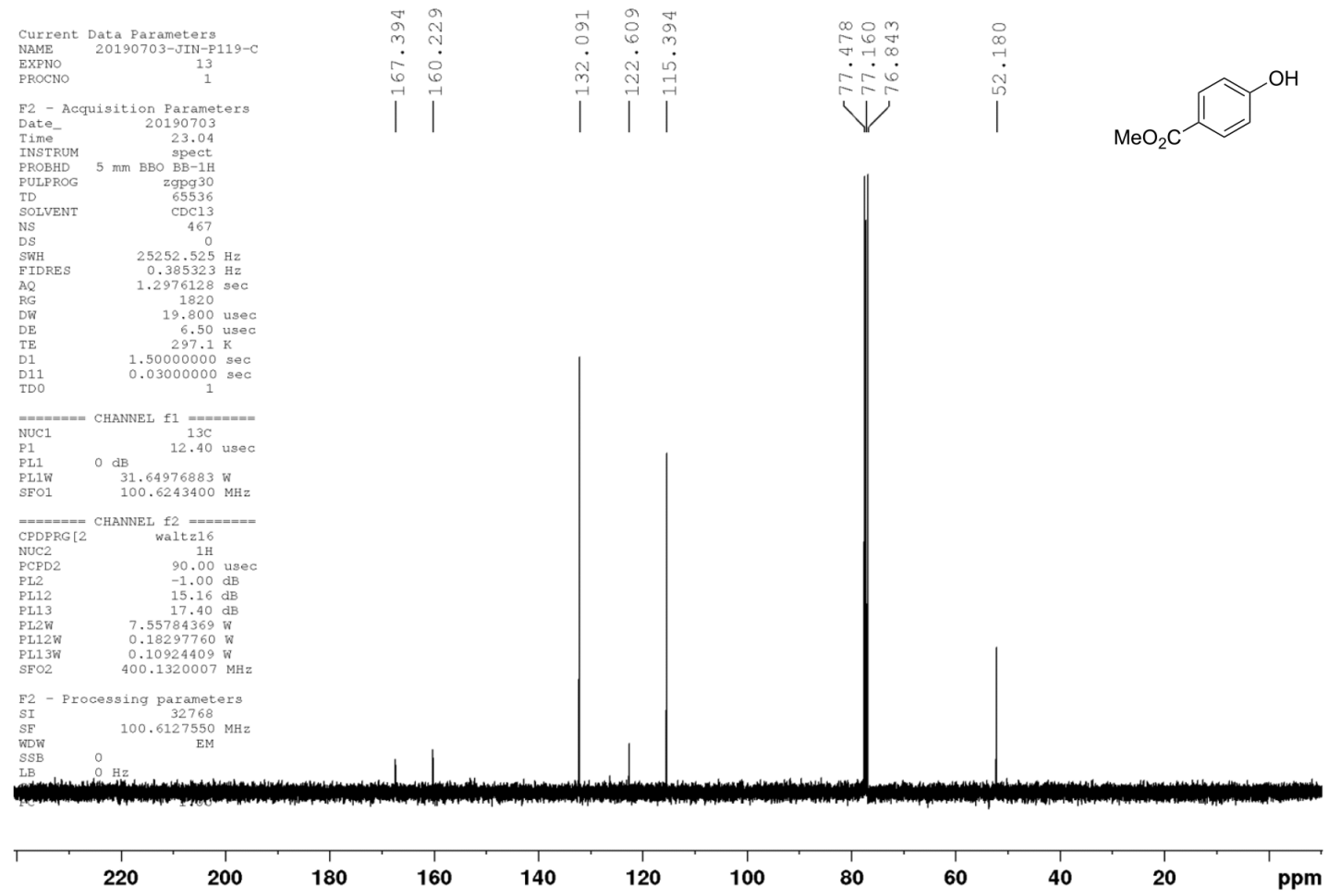

${ }^{1} \mathrm{H}$ NMR of $6 \mathrm{j}$, DMSO-d $6,400 \mathrm{MHz}, 21^{\circ} \mathrm{C}$
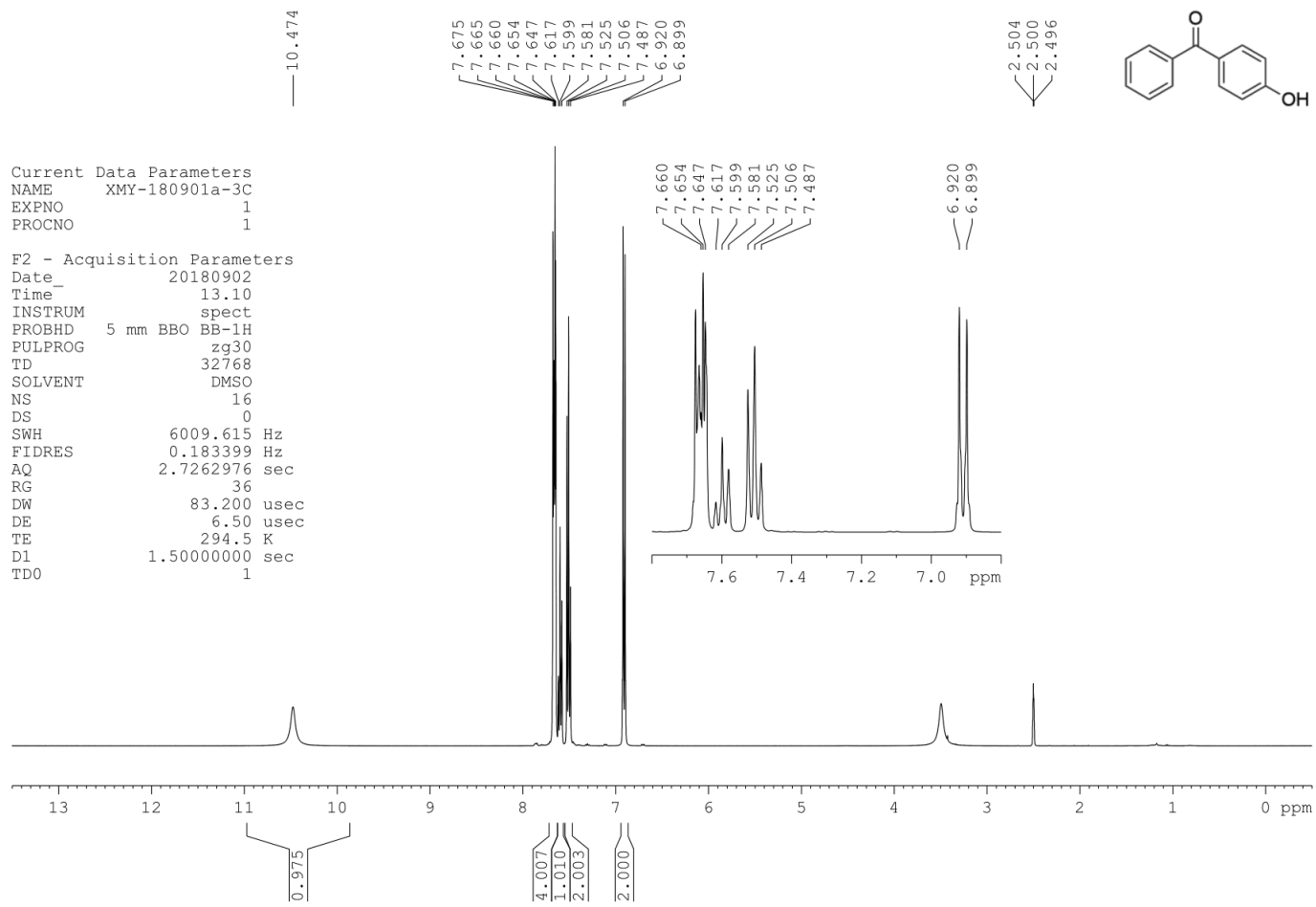
${ }^{13} \mathrm{C}$ NMR of $6 \mathrm{j}$, DMSO-d $6,100 \mathrm{MHz}, 22{ }^{\circ} \mathrm{C}$

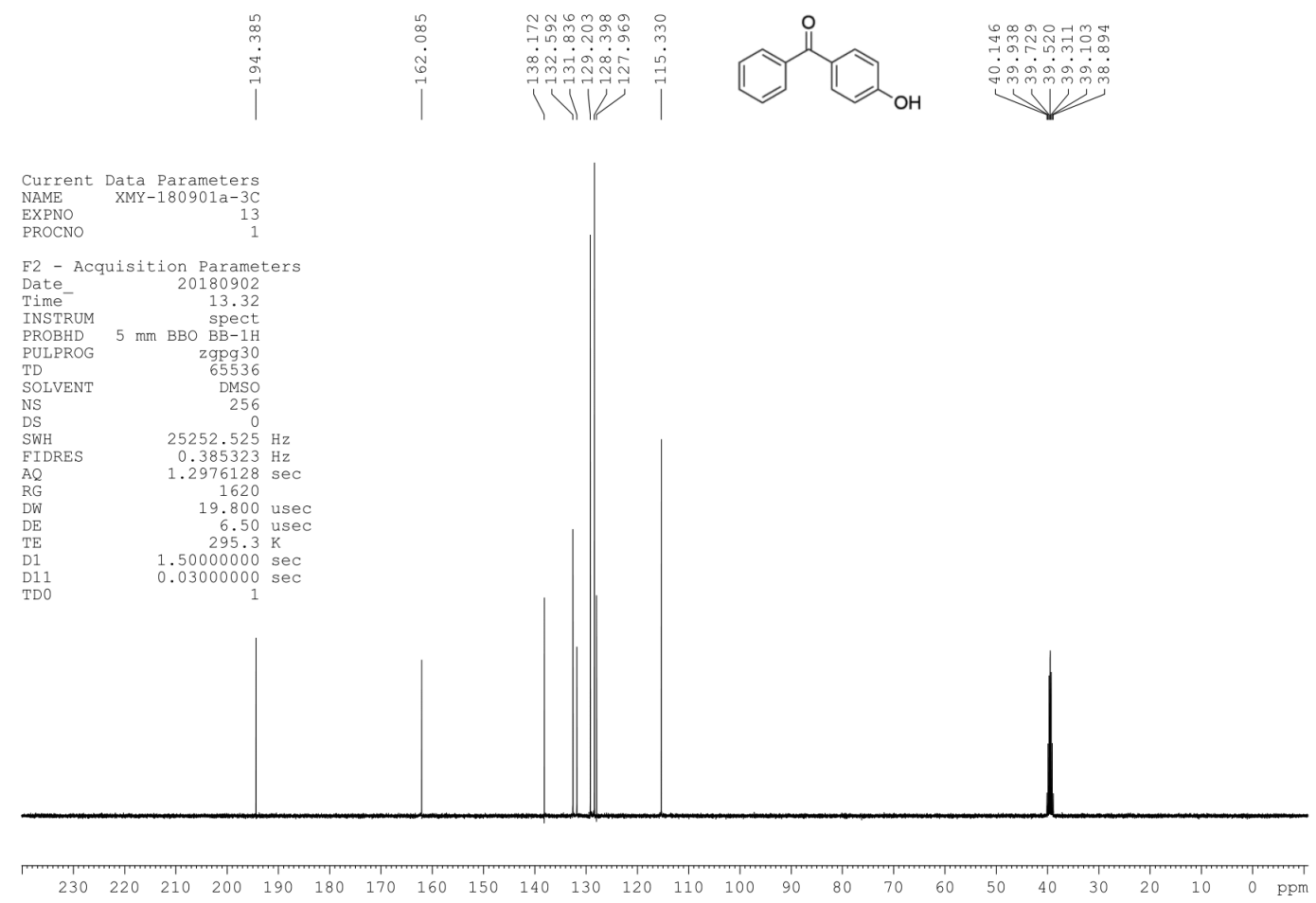

\section{${ }^{1} \mathrm{H}$ NMR of $6 \mathrm{k}, \mathrm{CDCl}_{3}, 400 \mathrm{MHz}, 21^{\circ} \mathrm{C}$}
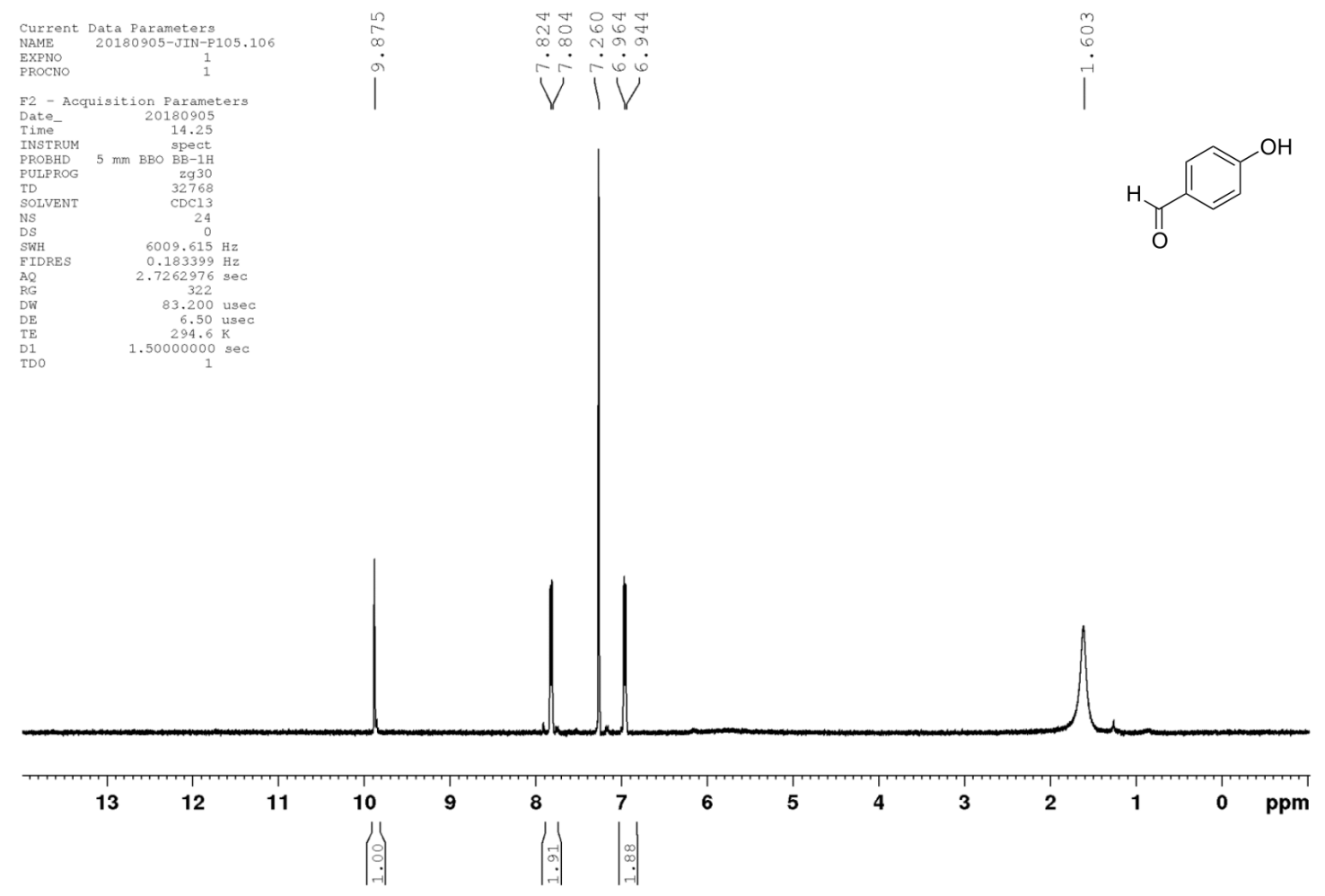
${ }^{13} \mathrm{C}$ NMR of $6 \mathrm{k}, \mathrm{CDCl}_{3}, 100 \mathrm{MHz}, 25{ }^{\circ} \mathrm{C}$

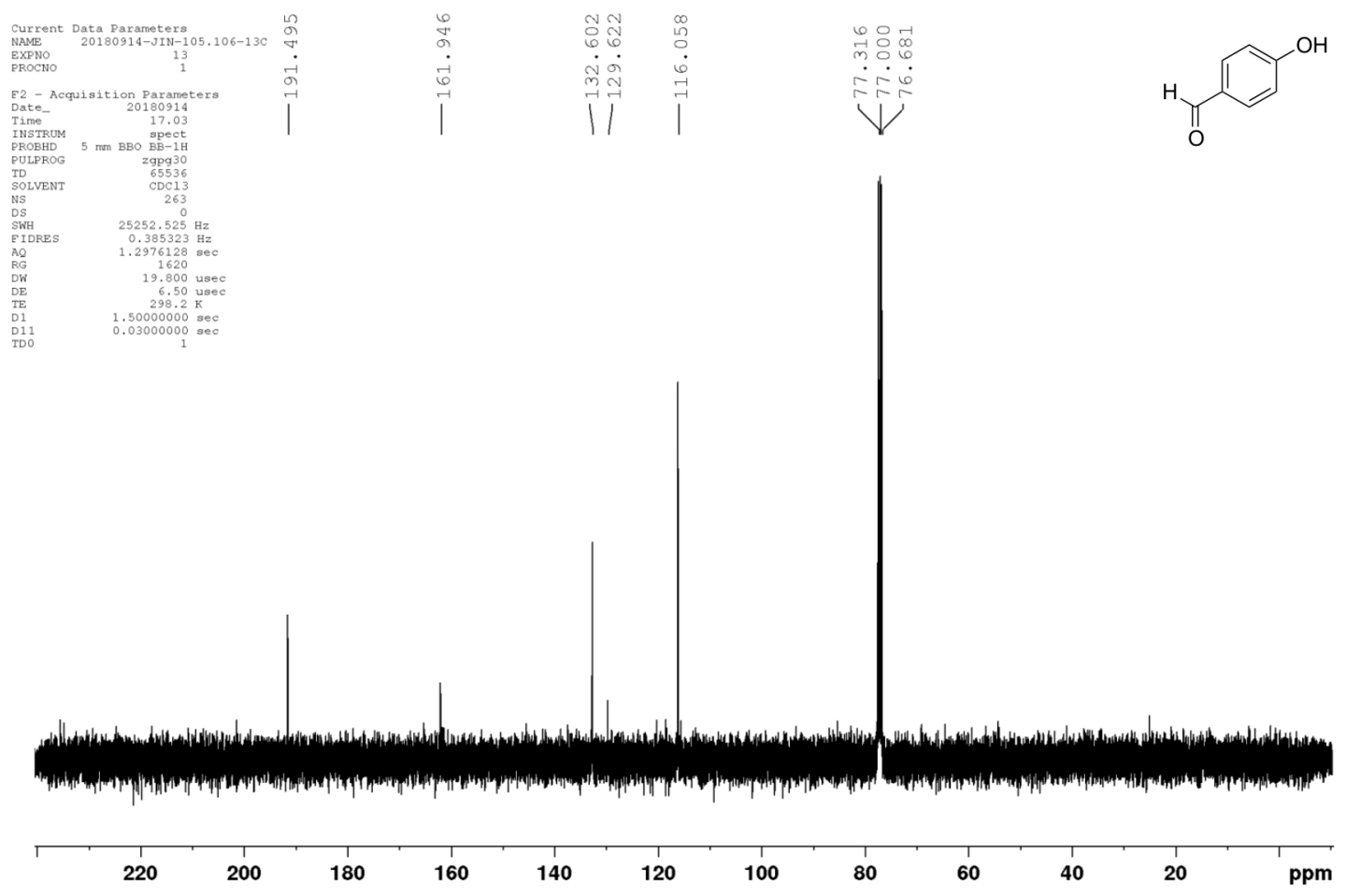

${ }^{1} \mathrm{H}$ NMR of $6 \mathrm{I}, \mathrm{CDCl}_{3}, 400 \mathrm{MHz}, 21^{\circ} \mathrm{C}$
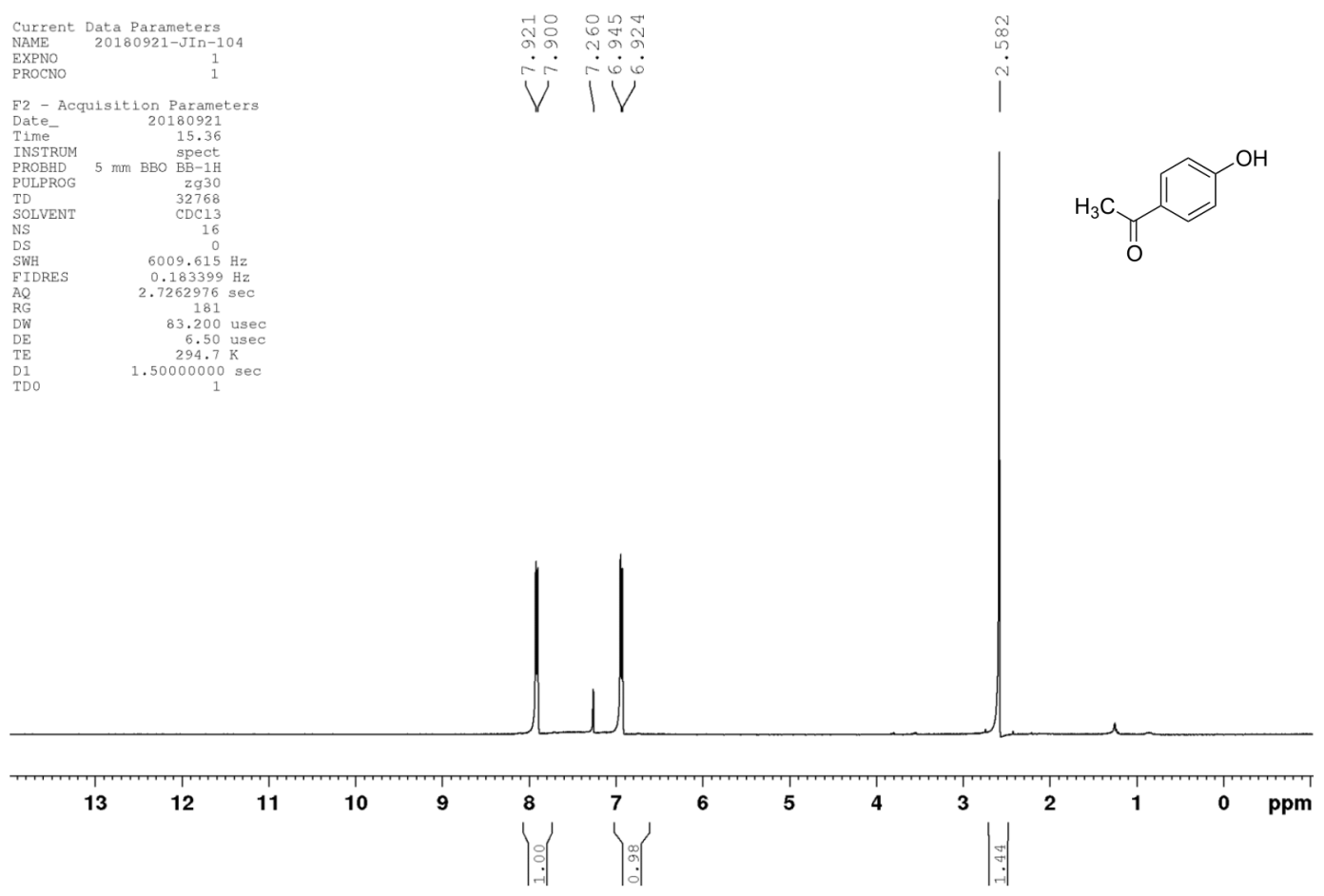
${ }^{13} \mathrm{C}$ NMR of $6 \mathrm{I}, \mathrm{CDCl}_{3}, 100 \mathrm{MHz}, 25{ }^{\circ} \mathrm{C}$
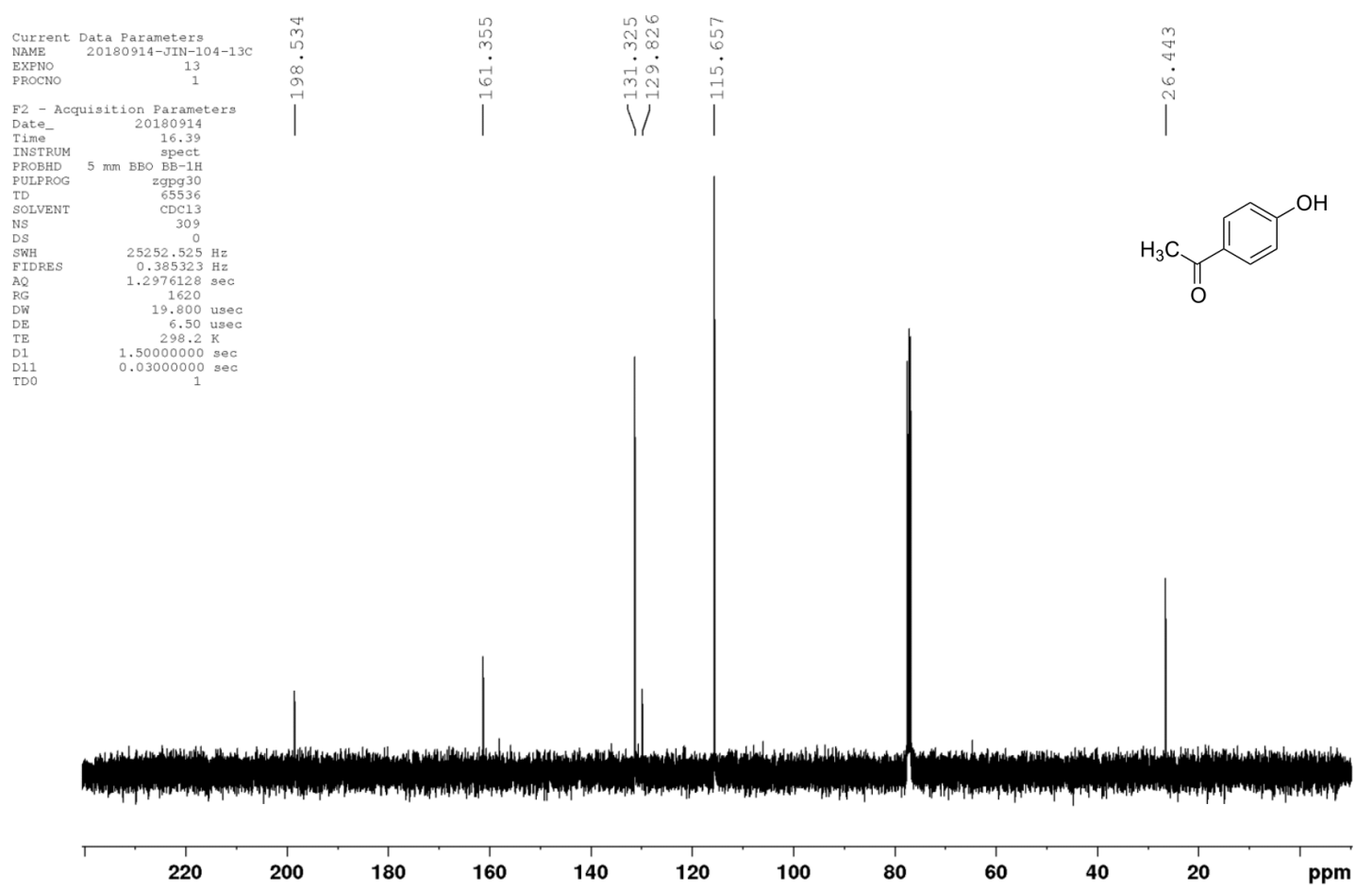

${ }^{1} \mathrm{H}$ NMR of $6 \mathrm{~m}$, DMSO-d $6,400 \mathrm{MHz}, 21^{\circ} \mathrm{C}$

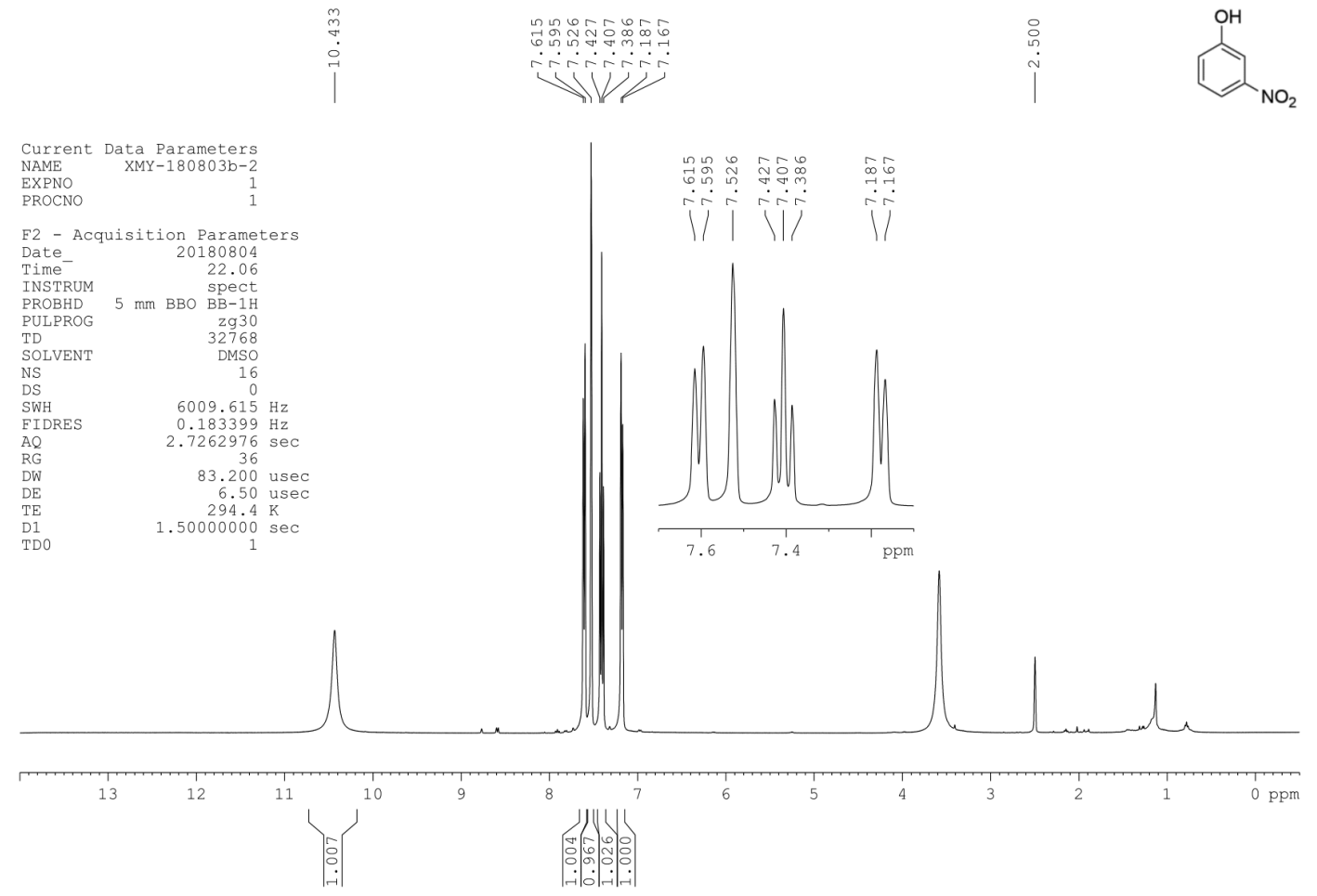




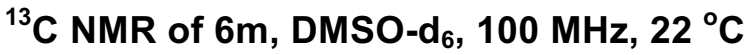
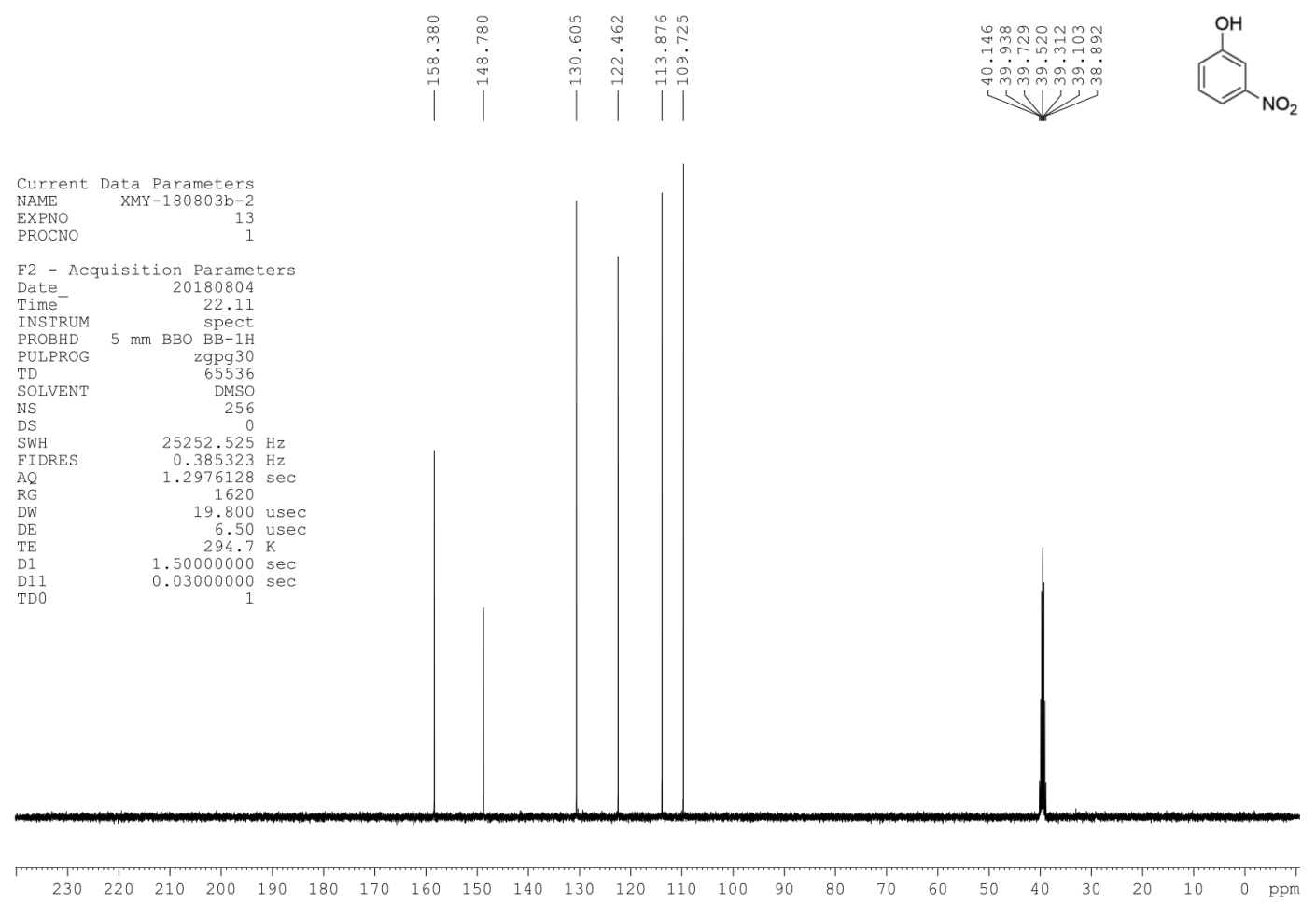

${ }^{1} \mathrm{H}$ NMR of $6 \mathrm{n}, \mathrm{CDCl}_{3}, 400 \mathrm{MHz}, 22^{\circ} \mathrm{C}$

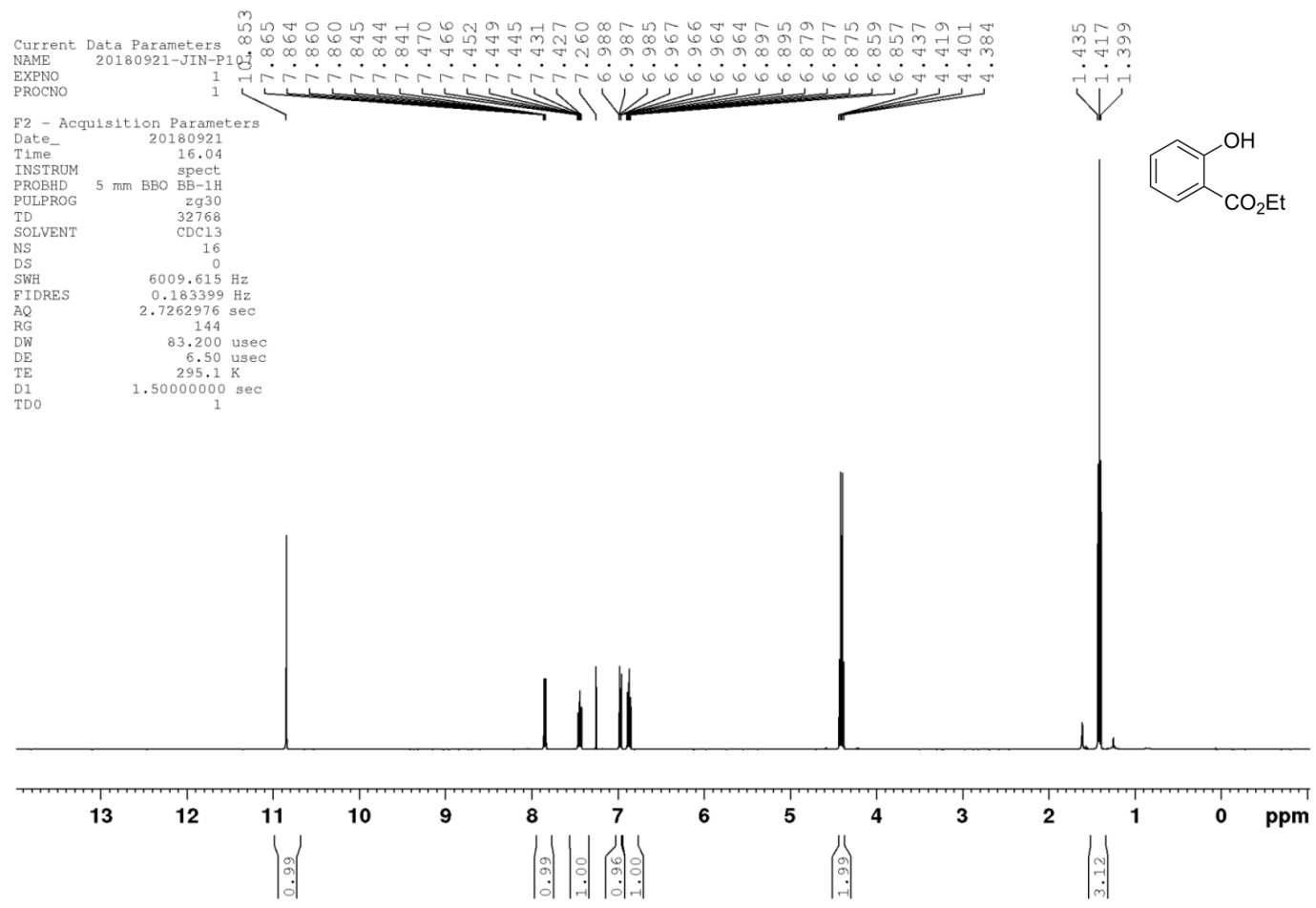


${ }^{13} \mathrm{C}$ NMR of $6 \mathrm{n}, \mathrm{CDCl}_{3}, 100 \mathrm{MHz}, 25^{\circ} \mathrm{C}$
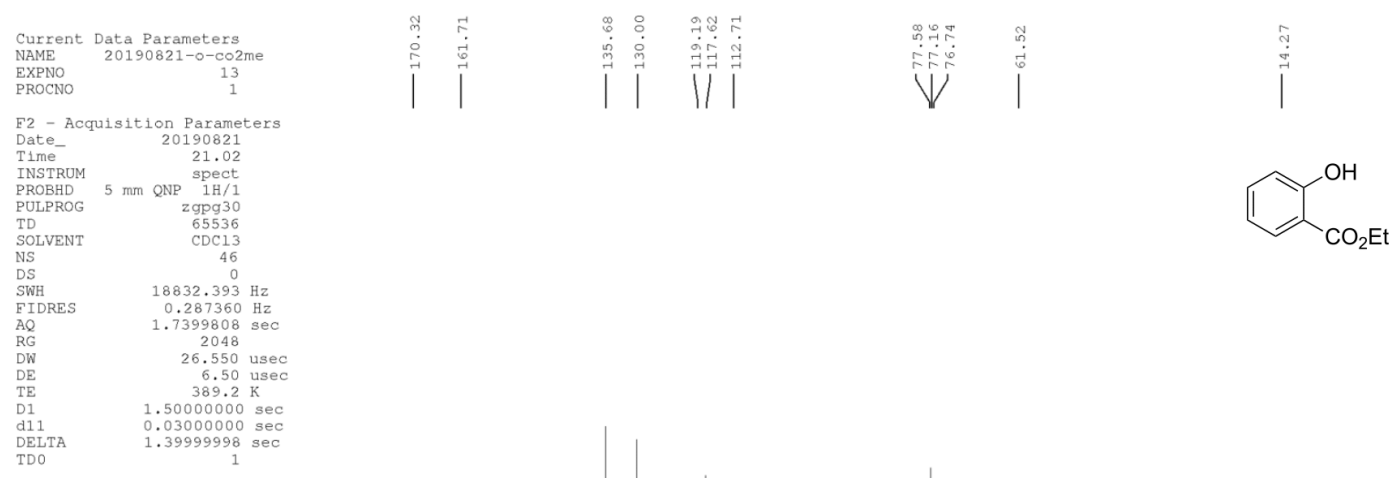

$\underset{1 D O}{\text { DELTA }} \quad 1.39999998 \mathrm{sec}$

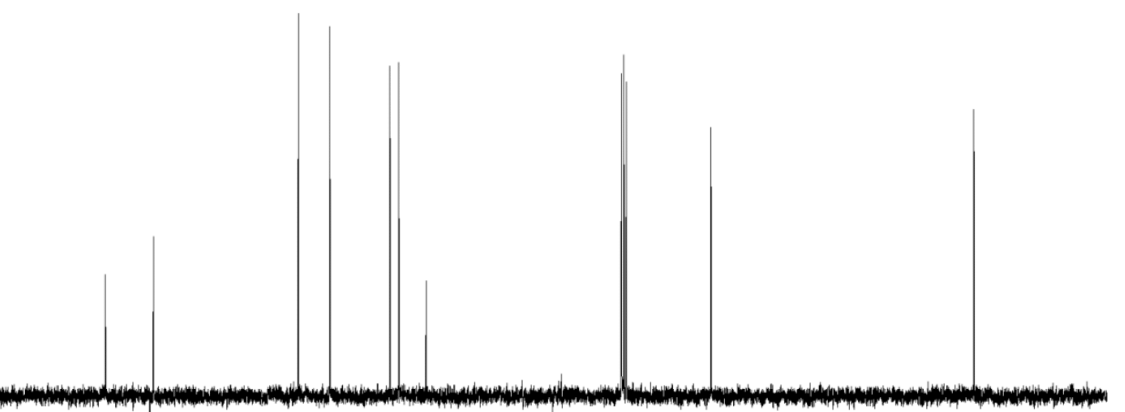

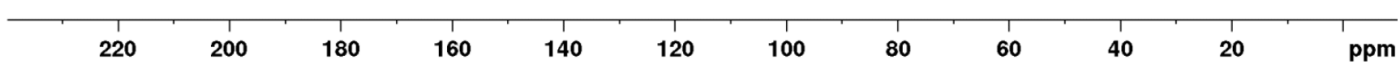

${ }^{1} \mathrm{H}$ NMR of $60, \mathrm{CDCl}_{3}, 400 \mathrm{MHz}, 21^{\circ} \mathrm{C}$

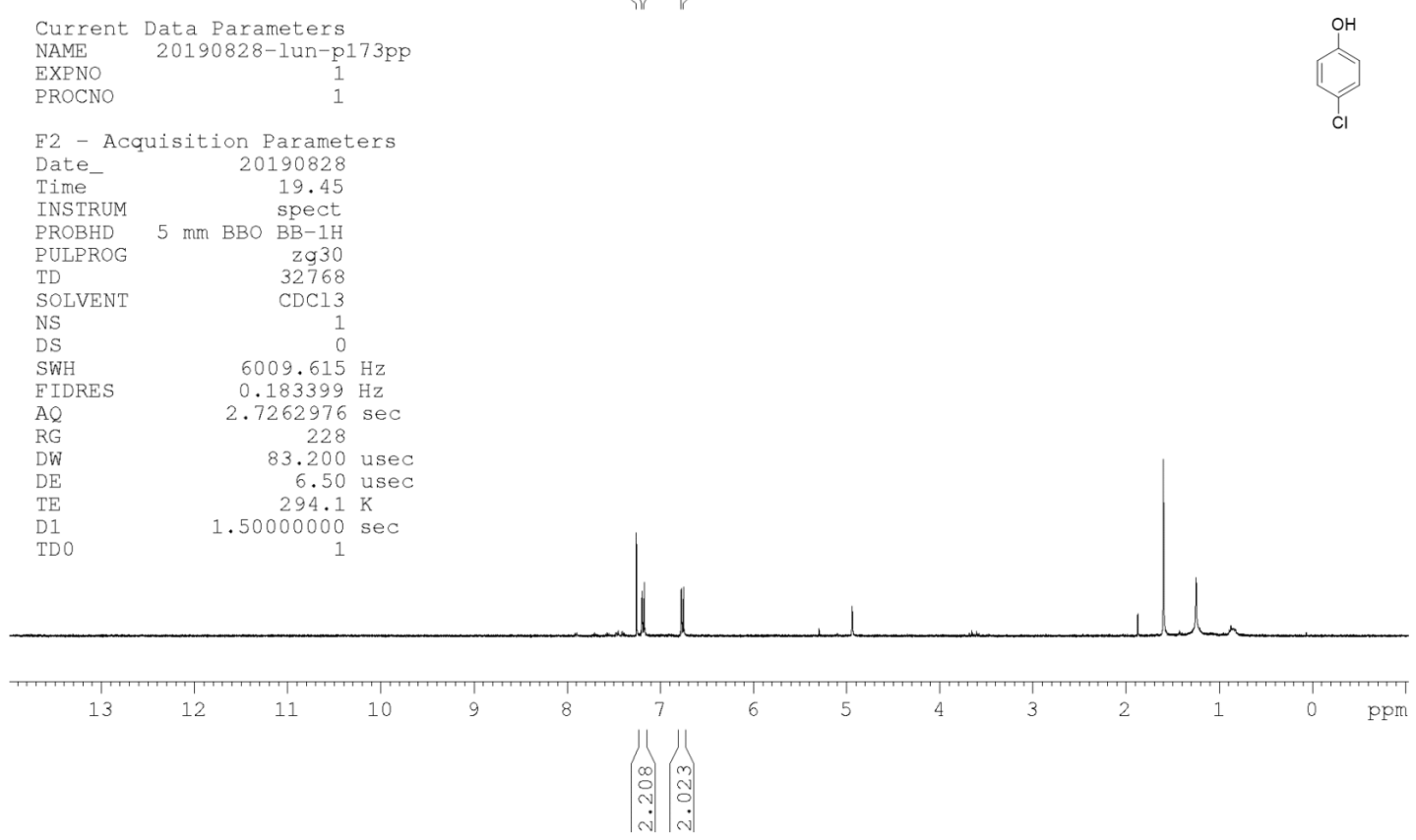


${ }^{13} \mathrm{C}$ NMR of $60, \mathrm{CDCl}_{3}, 100 \mathrm{MHz}, 25^{\circ} \mathrm{C}$

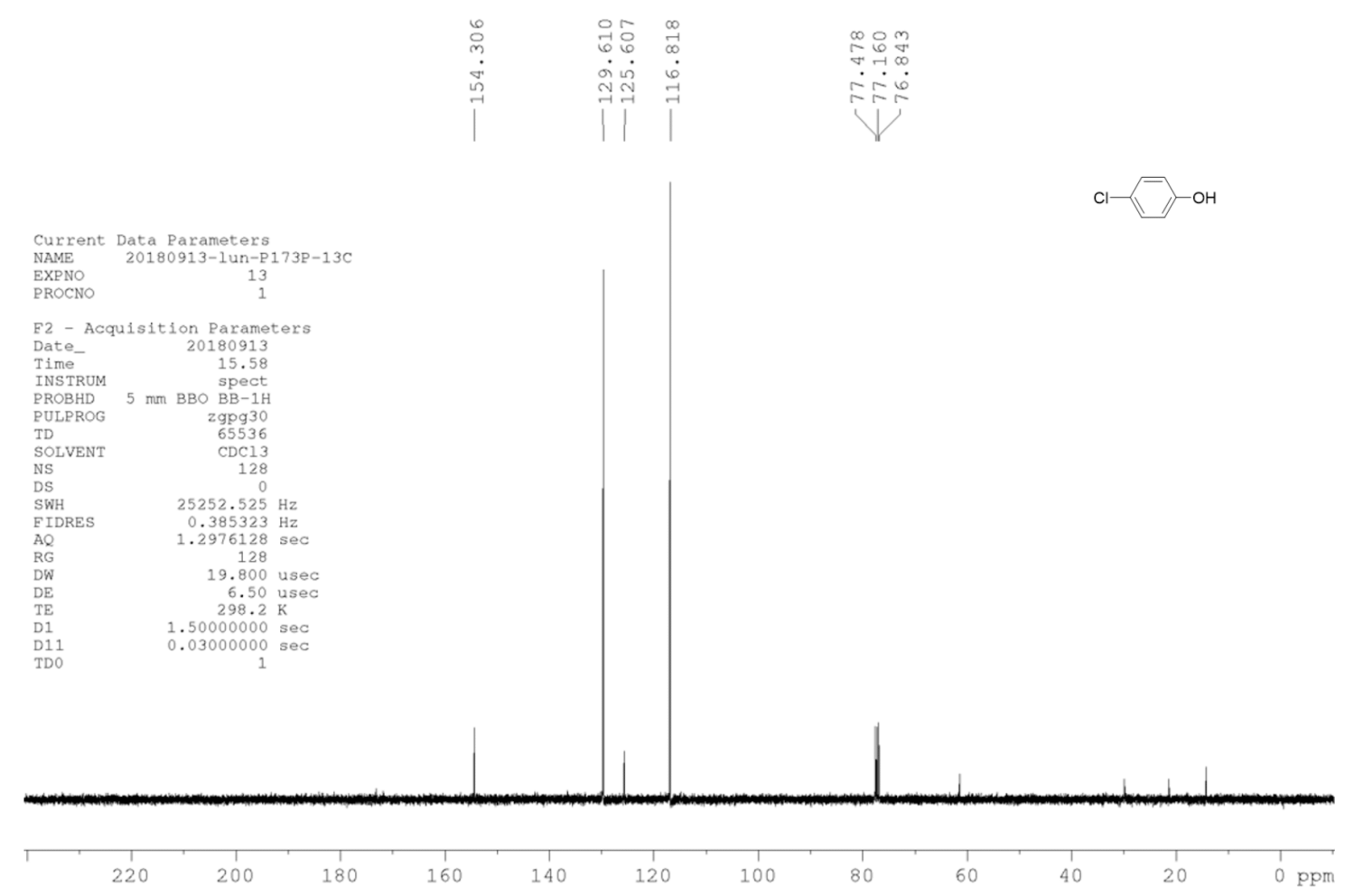

${ }^{1} \mathrm{H}$ NMR of $6 \mathrm{p}, \mathrm{CDCl}_{3}, 400 \mathrm{MHz}, 21^{\circ} \mathrm{C}$

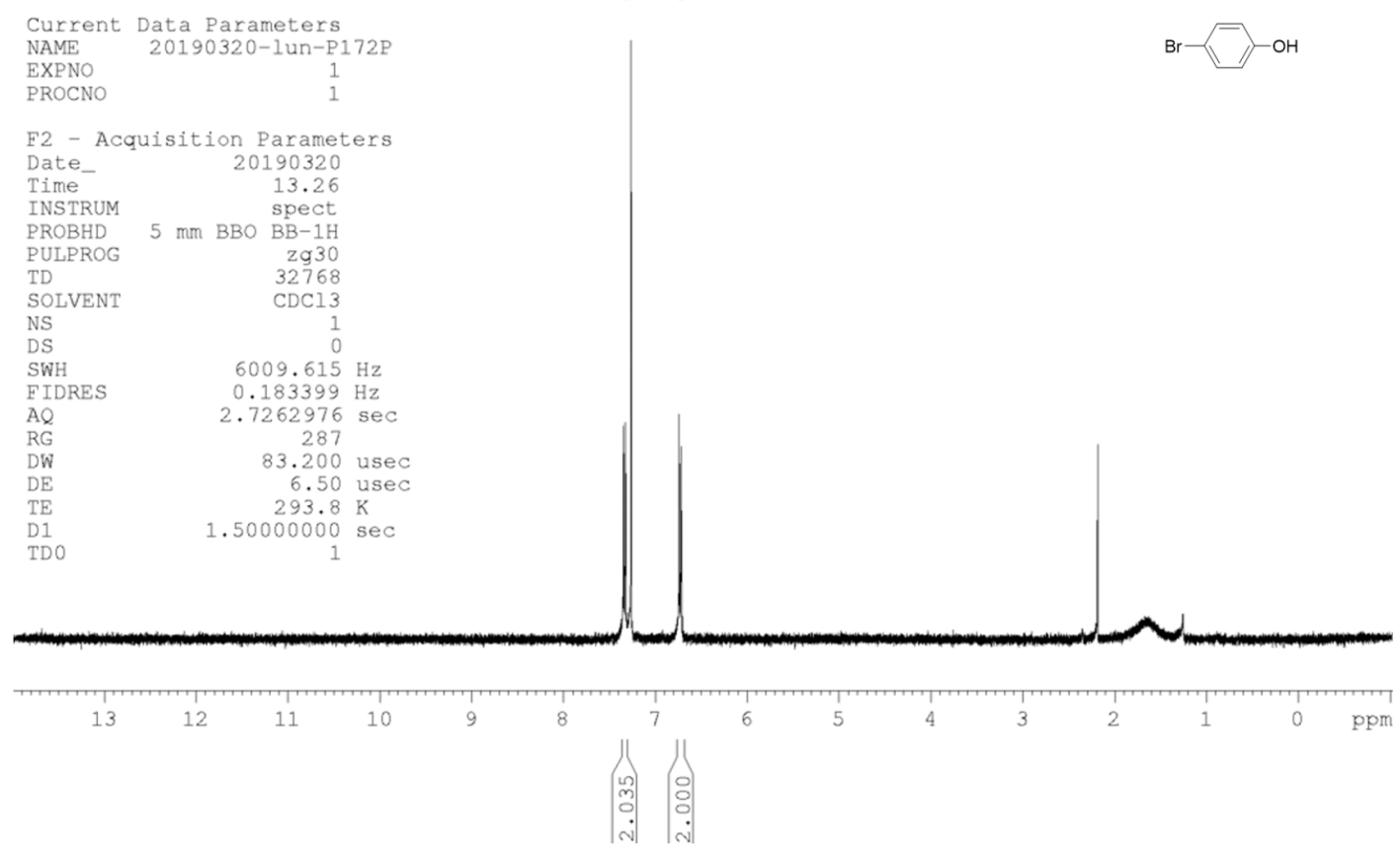


${ }^{13} \mathrm{C}$ NMR of $6 \mathrm{p}, \mathrm{CDCl}_{3}, 100 \mathrm{MHz}, 25^{\circ} \mathrm{C}$

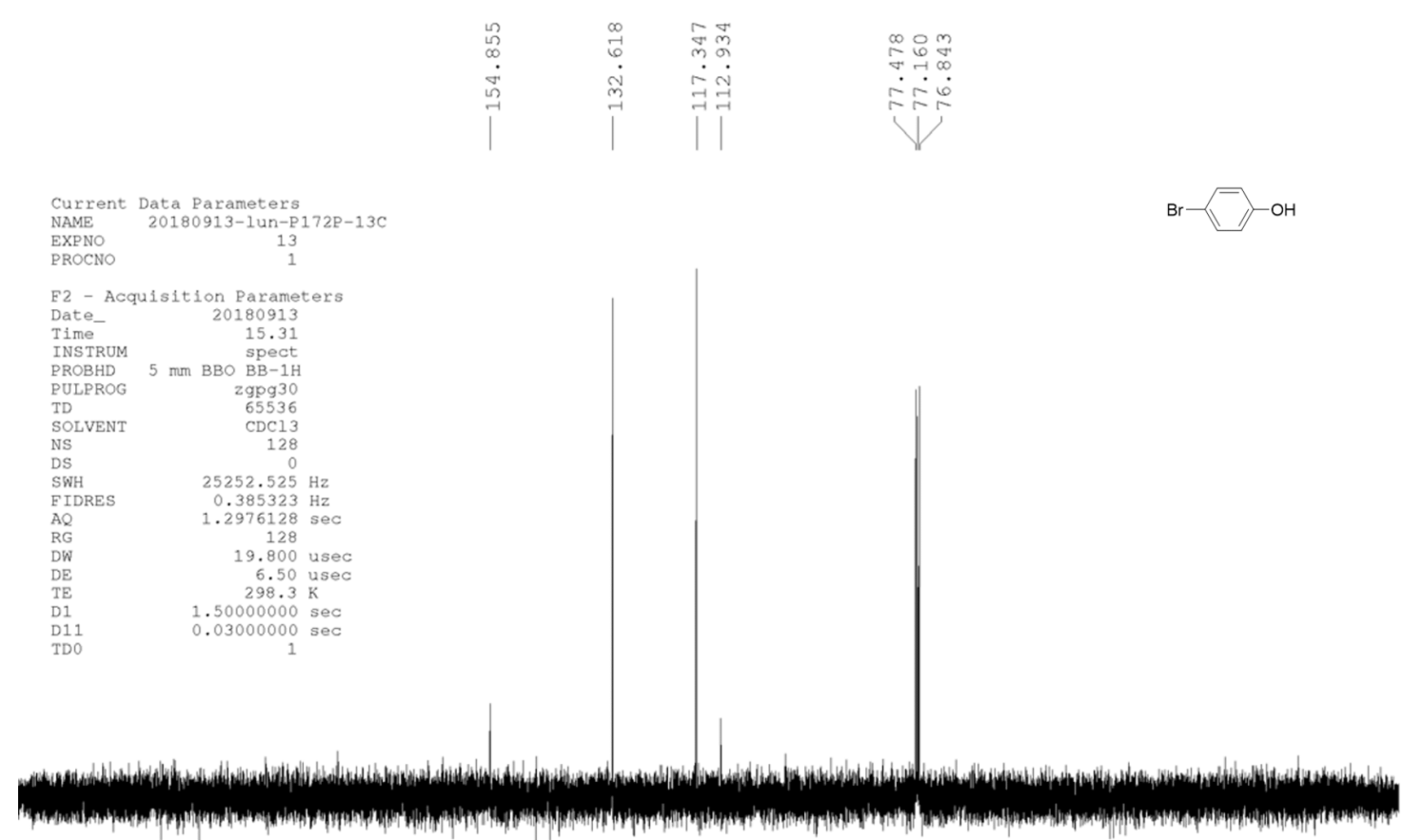

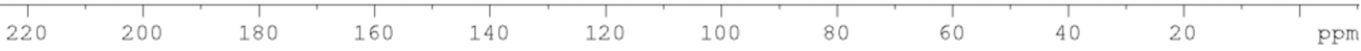

${ }^{1} \mathrm{H}$ NMR of $6 \mathrm{q}, \mathrm{CDCl}_{3}, 400 \mathrm{MHz}, 21^{\circ} \mathrm{C}$

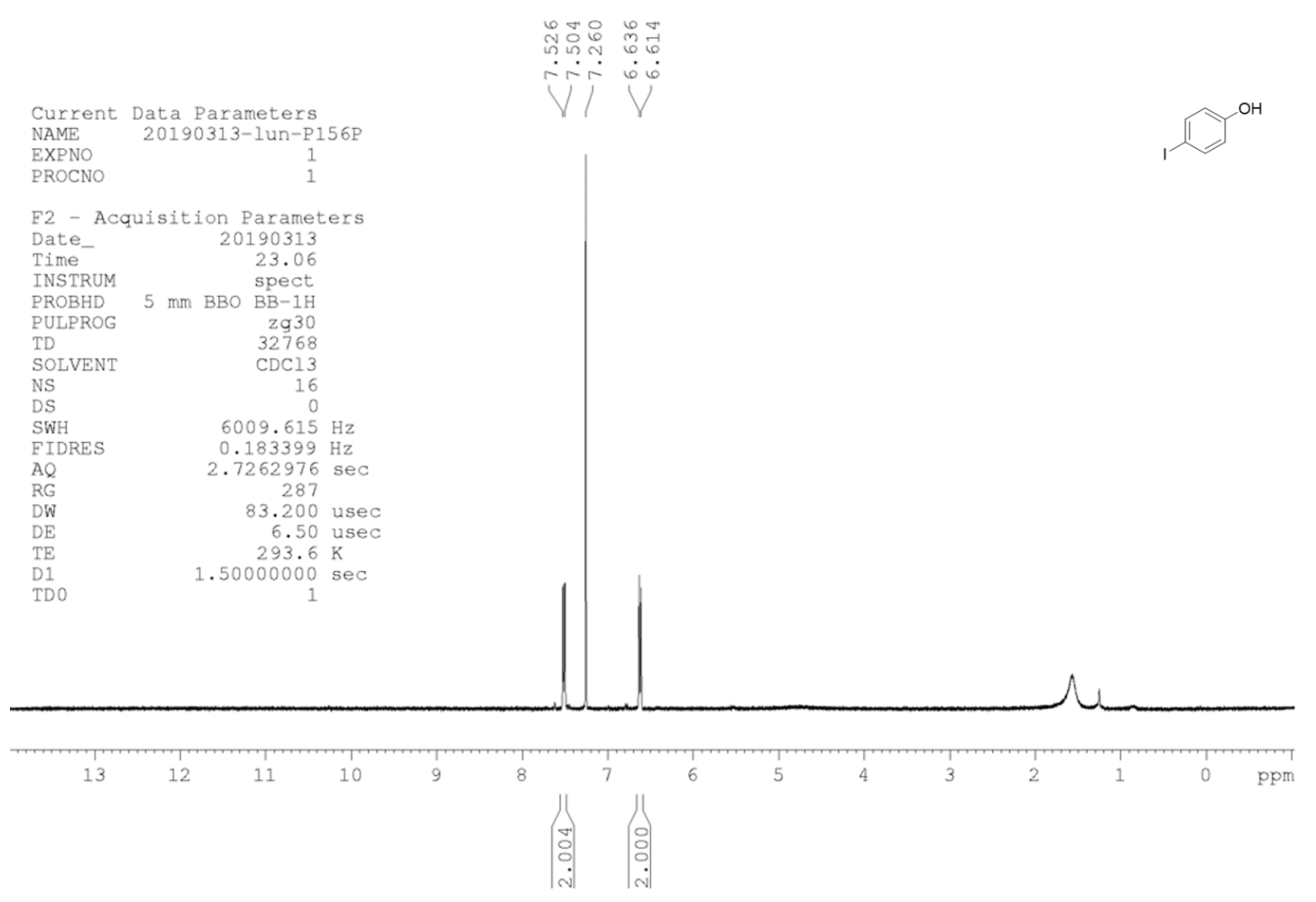


${ }^{13} \mathrm{C}$ NMR of $6 \mathrm{q}, \mathrm{CDCl}_{3}, 100 \mathrm{MHz}, 25{ }^{\circ} \mathrm{C}$

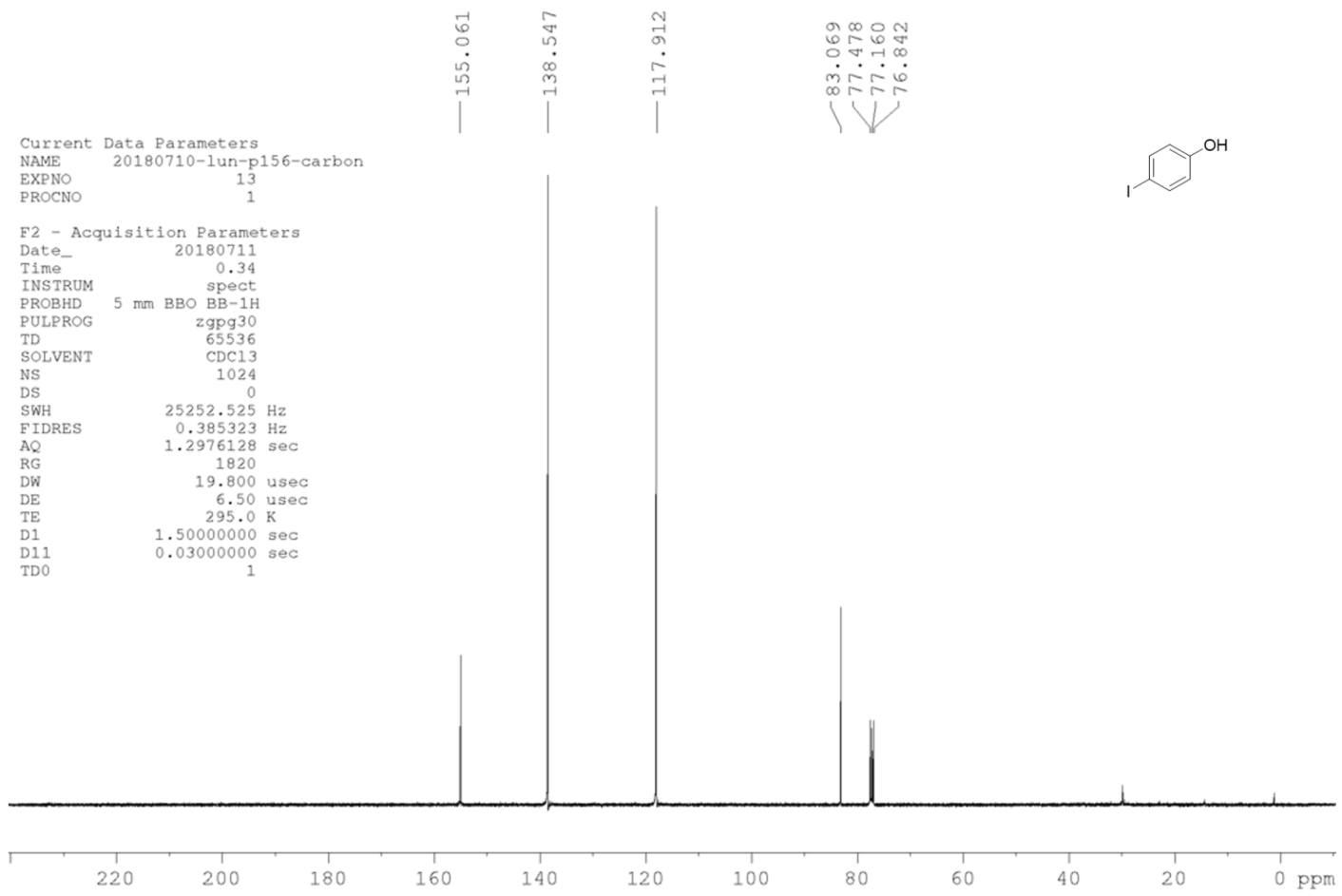

${ }^{1} \mathrm{H}$ NMR of $6 \mathrm{r}, \mathrm{CDCl}_{3}, 400 \mathrm{MHz}, 21^{\circ} \mathrm{C}$

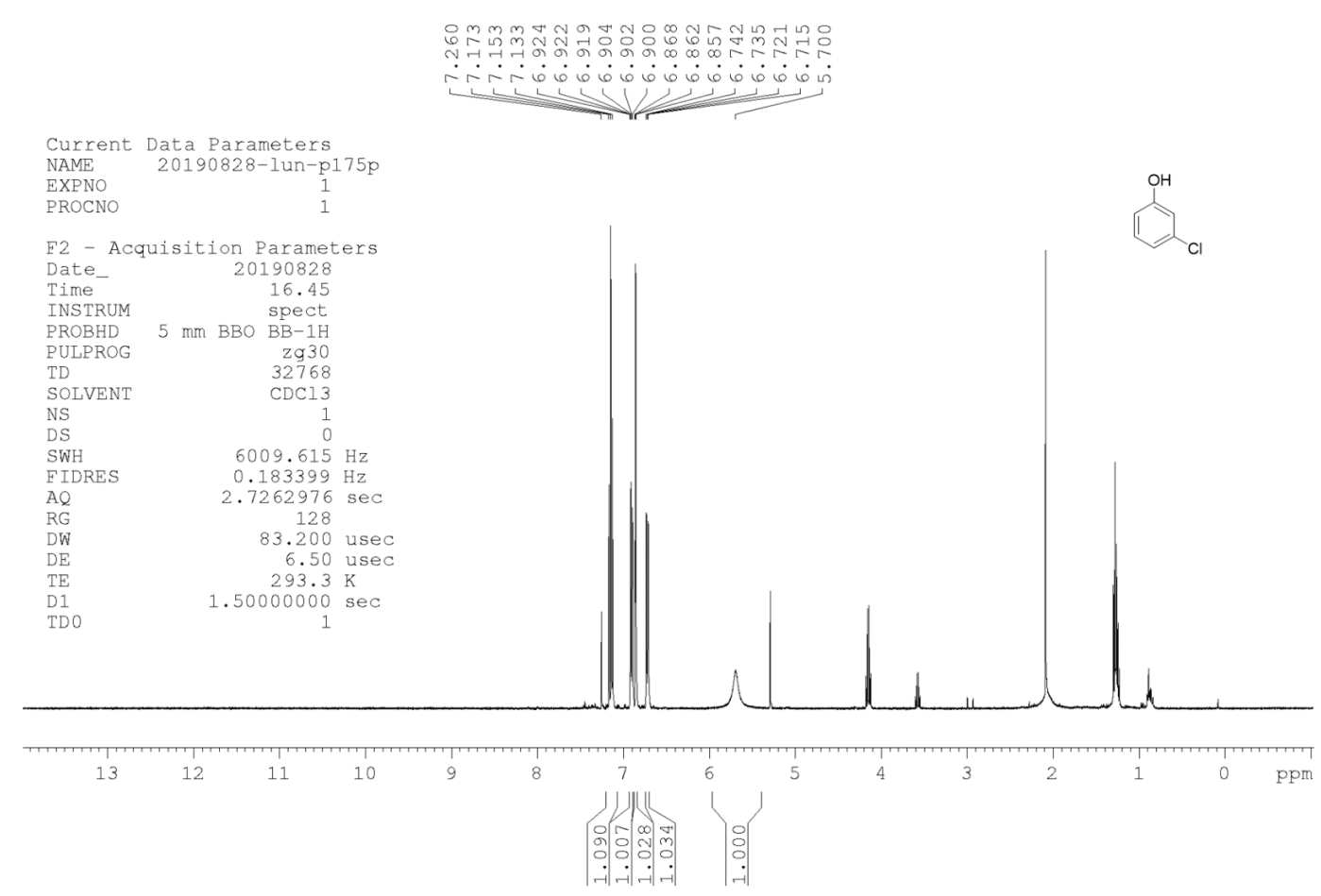

${ }^{*}$ Due to the volatility of product, EtOAc and ether are present in this spectrum. 
${ }^{13} \mathrm{C}$ NMR of $6 r, \mathrm{CDCl}_{3}, 100 \mathrm{MHz}, 25^{\circ} \mathrm{C}$
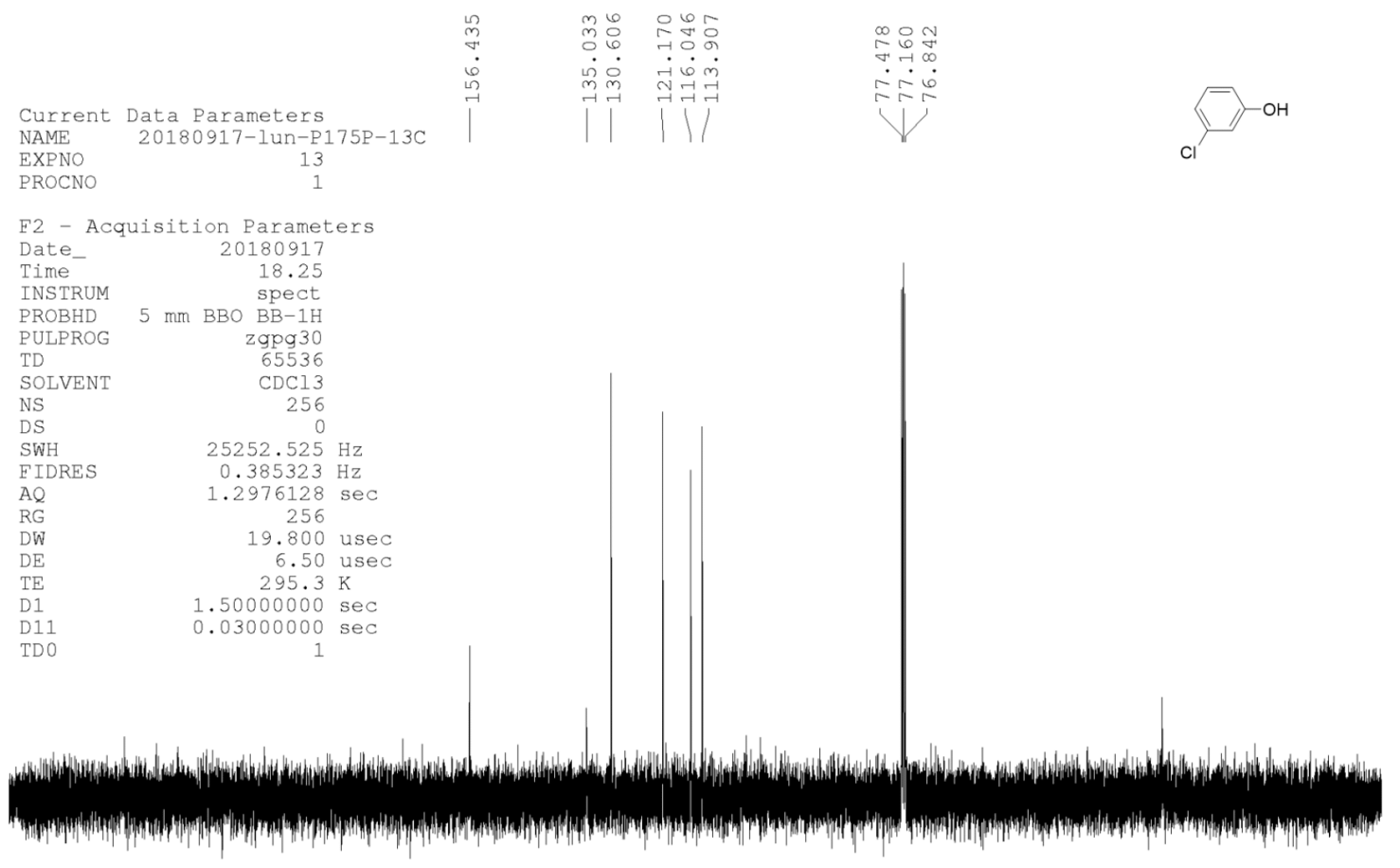

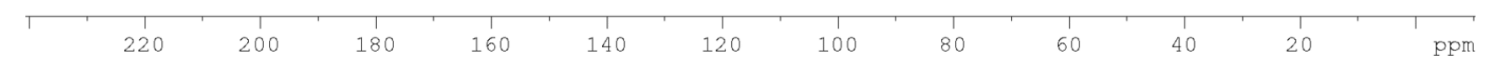

\section{${ }^{1} \mathrm{H}$ NMR of $6 \mathrm{~s}, \mathrm{CDCl}_{3}, 400 \mathrm{MHz}, 21^{\circ} \mathrm{C}$}
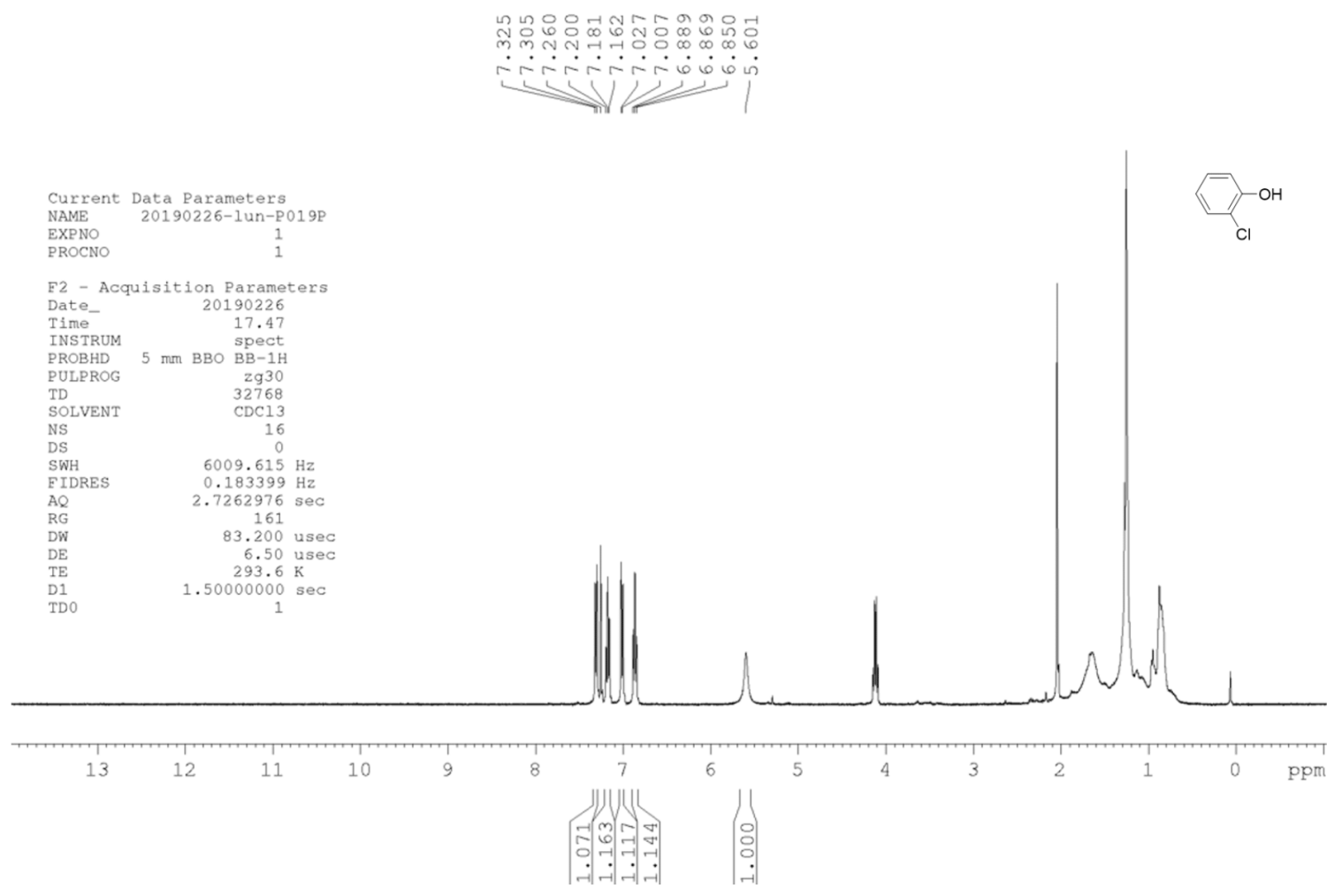
${ }^{13} \mathrm{C}$ NMR of $6 \mathrm{~s}, \mathrm{CDCl}_{3}, 100 \mathrm{MHz}, 25{ }^{\circ} \mathrm{C}$
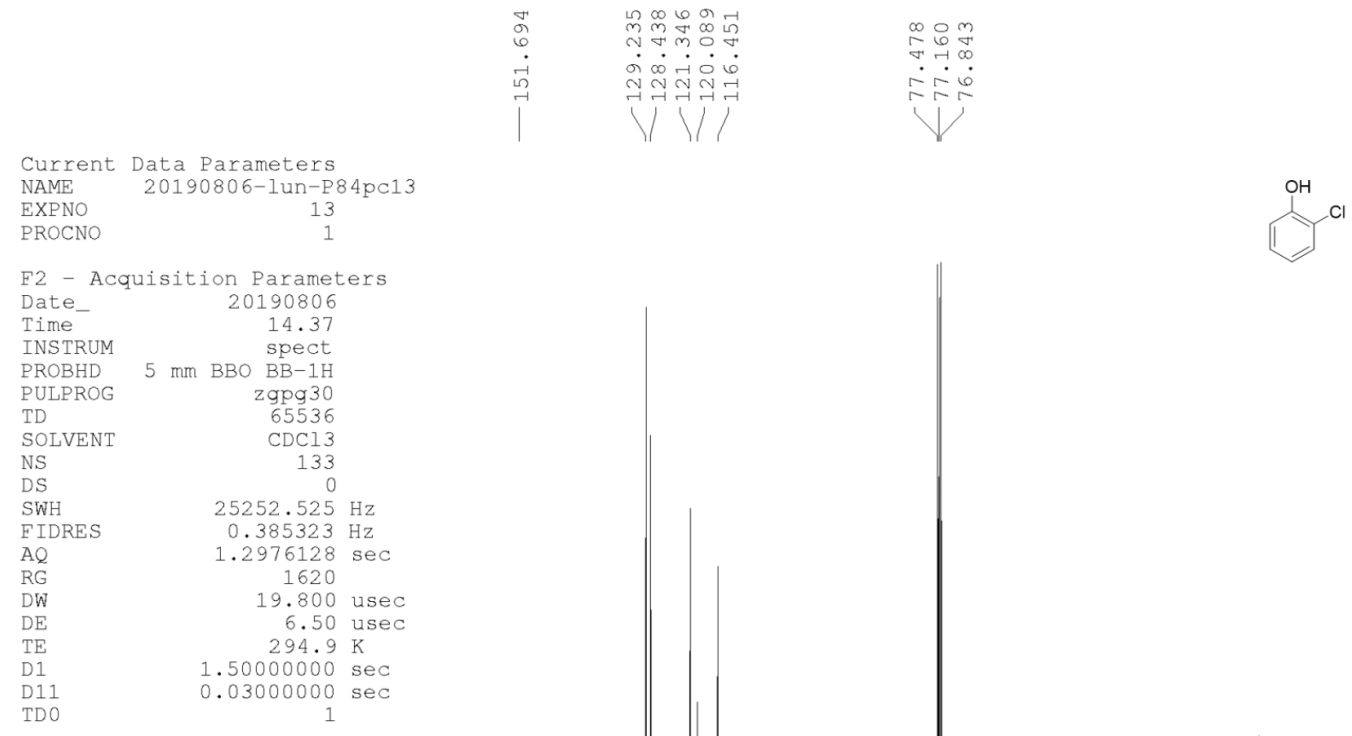

W.

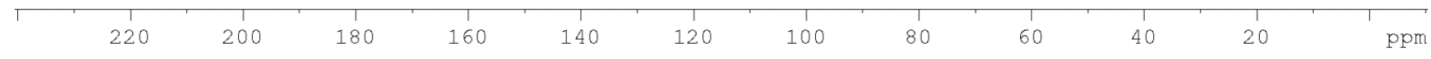

${ }^{1} \mathrm{H}$ NMR of $6 \mathrm{t}, \mathrm{CDCl}_{3}, 400 \mathrm{MHz}, 21^{\circ} \mathrm{C}$

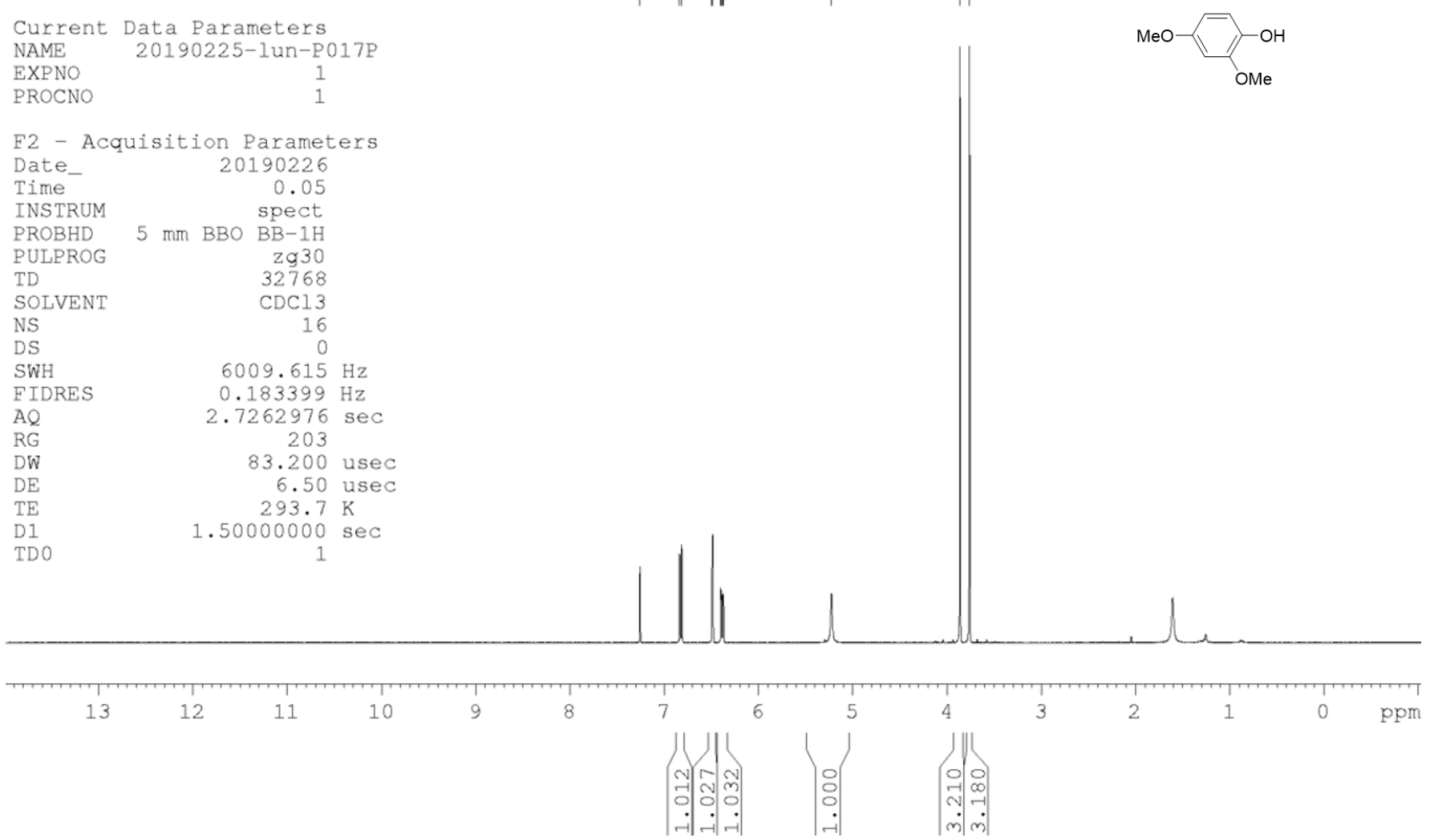


${ }^{13} \mathrm{C}$ NMR of $6 t, \mathrm{CDCl}_{3}, 100 \mathrm{MHz}, 25^{\circ} \mathrm{C}$

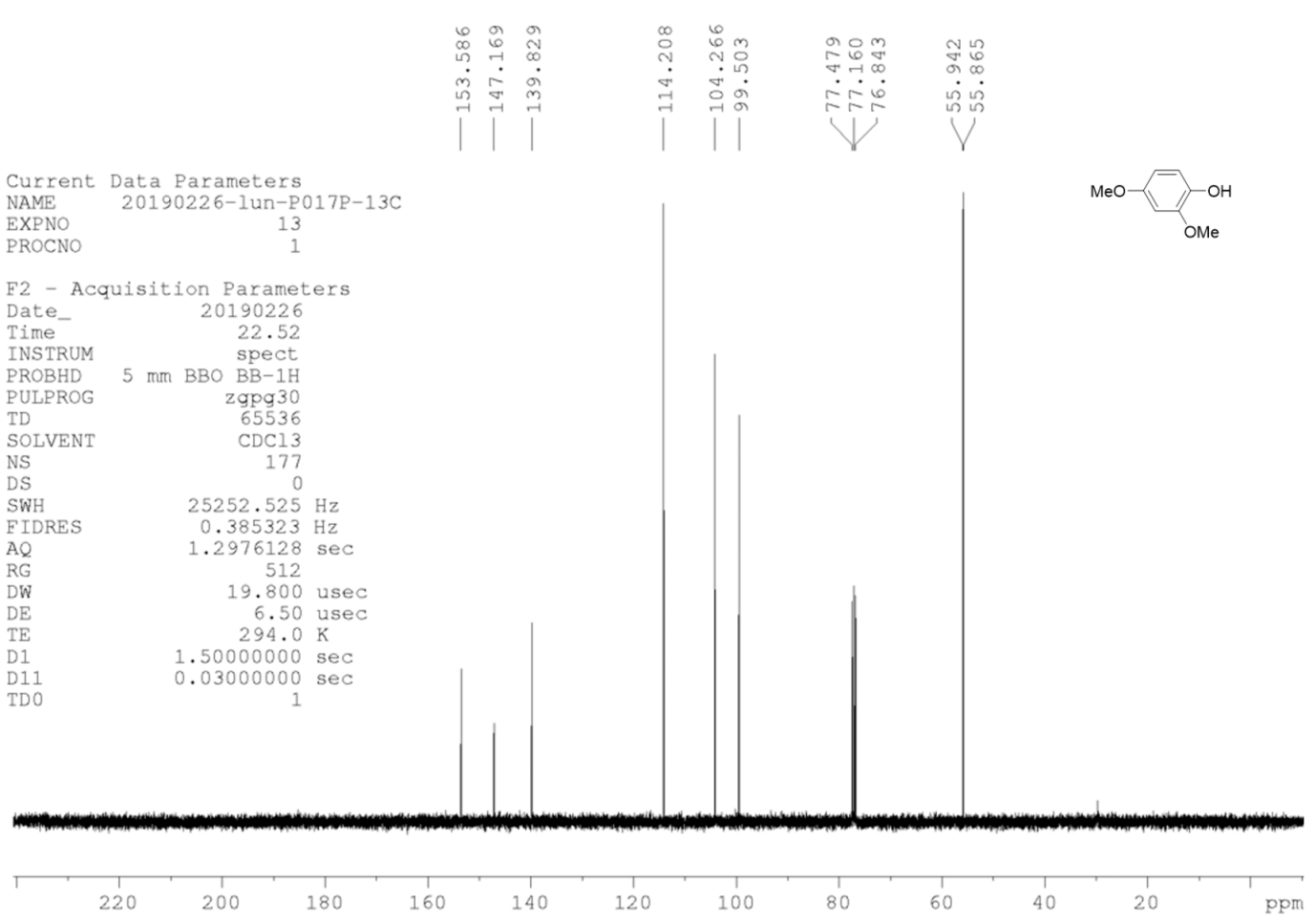

${ }^{1} \mathrm{H}$ NMR of $6 \mathrm{u}, \mathrm{CDCl}_{3}, 400 \mathrm{MHz}, 21^{\circ} \mathrm{C}$

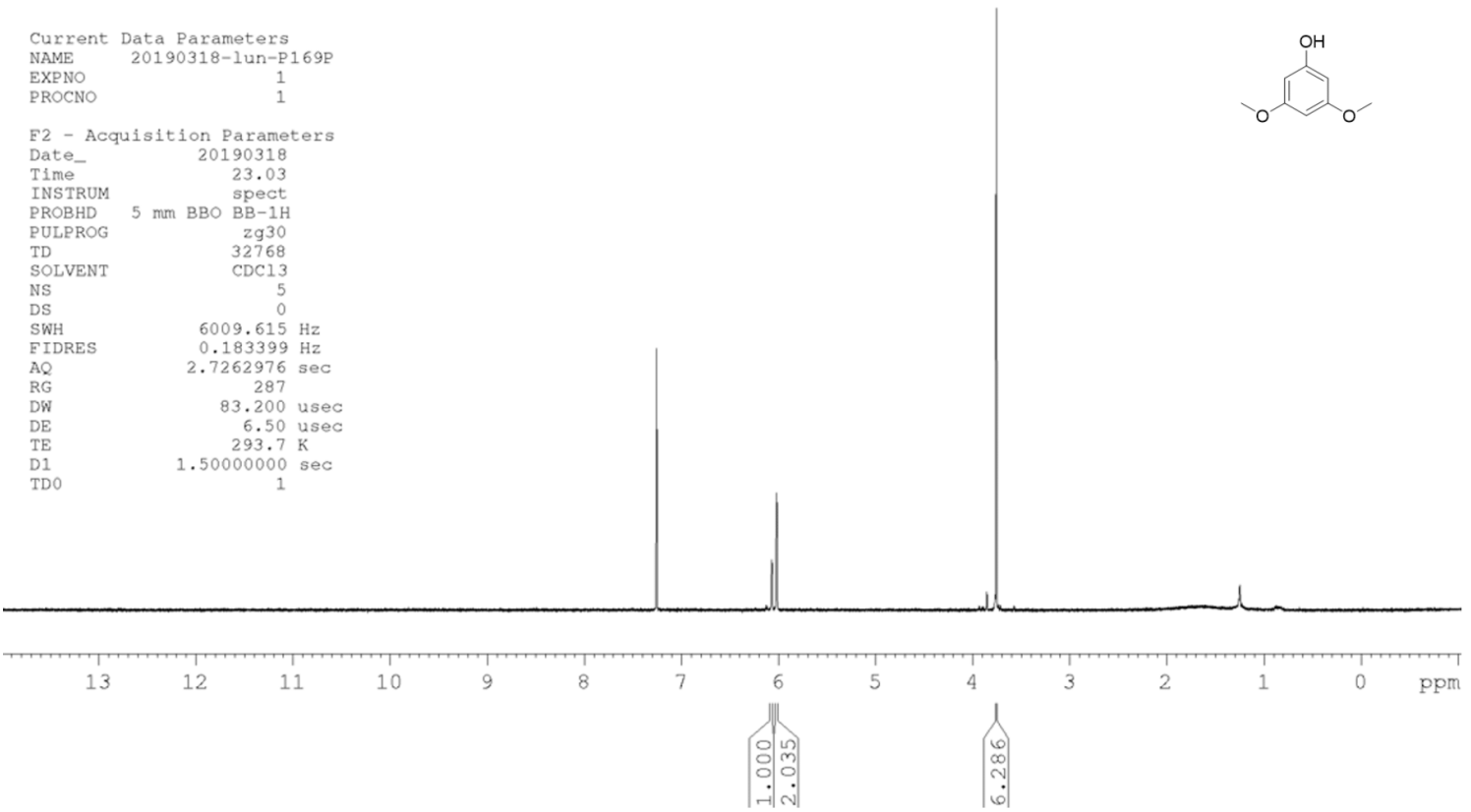


${ }^{13} \mathrm{C}$ NMR of $6 \mathrm{u}, \mathrm{CDCl}_{3}, 100 \mathrm{MHz}, 25^{\circ} \mathrm{C}$

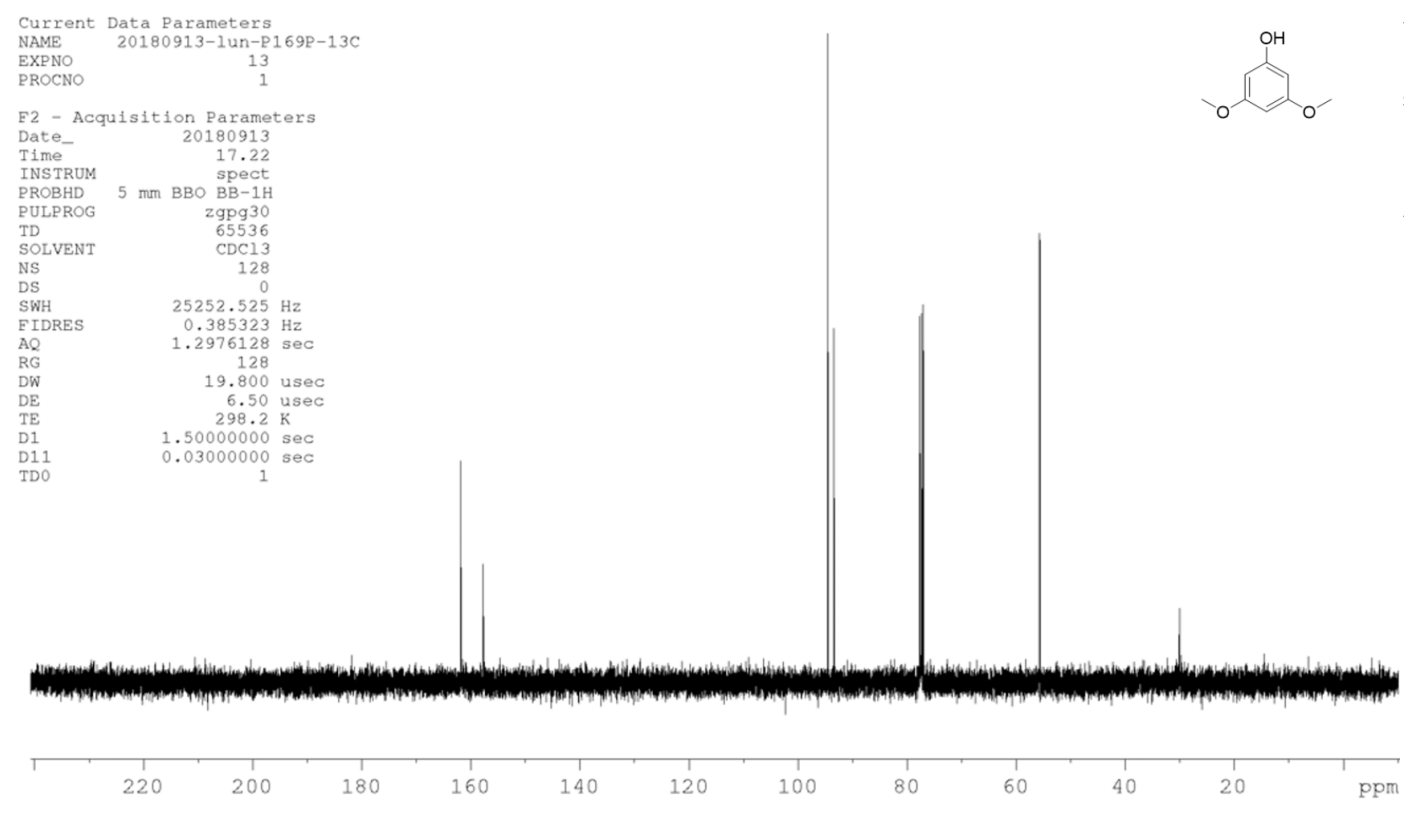

${ }^{1} \mathrm{H}$ NMR of $6 \mathrm{v}, \mathrm{CDCl}_{3}, 400 \mathrm{MHz}, 21^{\circ} \mathrm{C}$

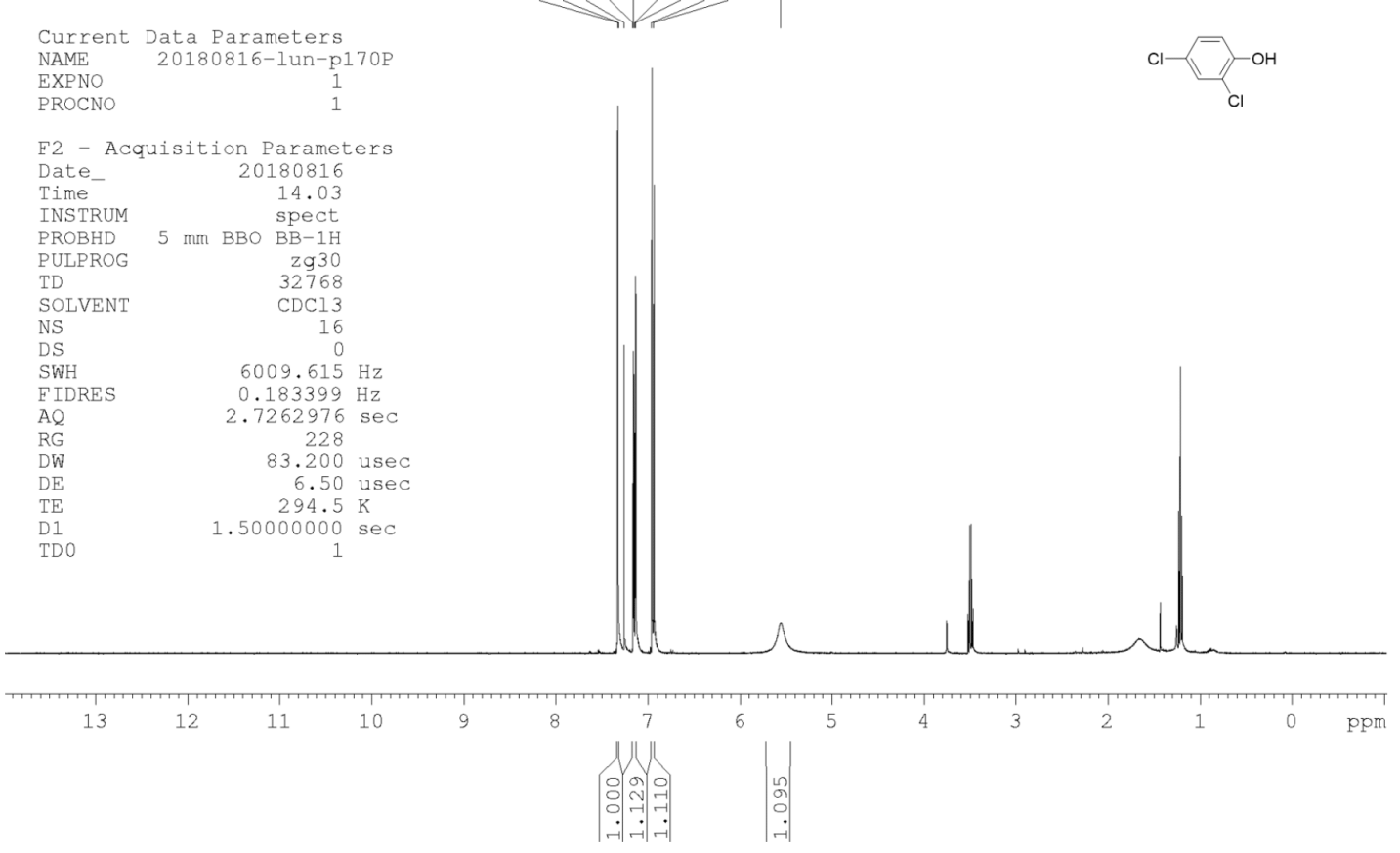


${ }^{13} \mathrm{C}$ NMR of $6 \mathrm{v}, \mathrm{CDCl}_{3}, 100 \mathrm{MHz}, 25{ }^{\circ} \mathrm{C}$

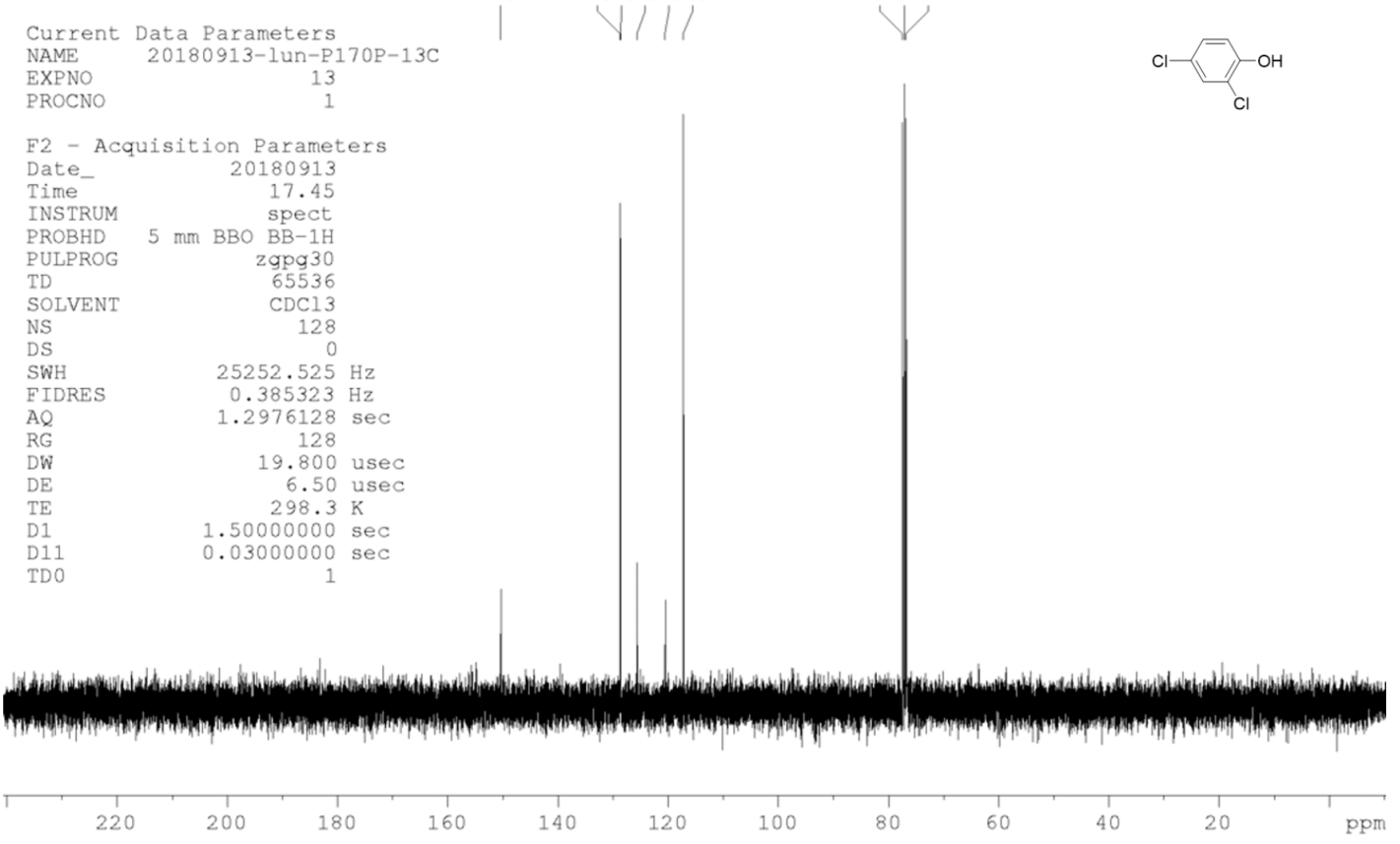

${ }^{1} \mathrm{H}$ NMR of $6 \mathrm{w}, \mathrm{CDCl}_{3}, 400 \mathrm{MHz}, 21^{\circ} \mathrm{C}$

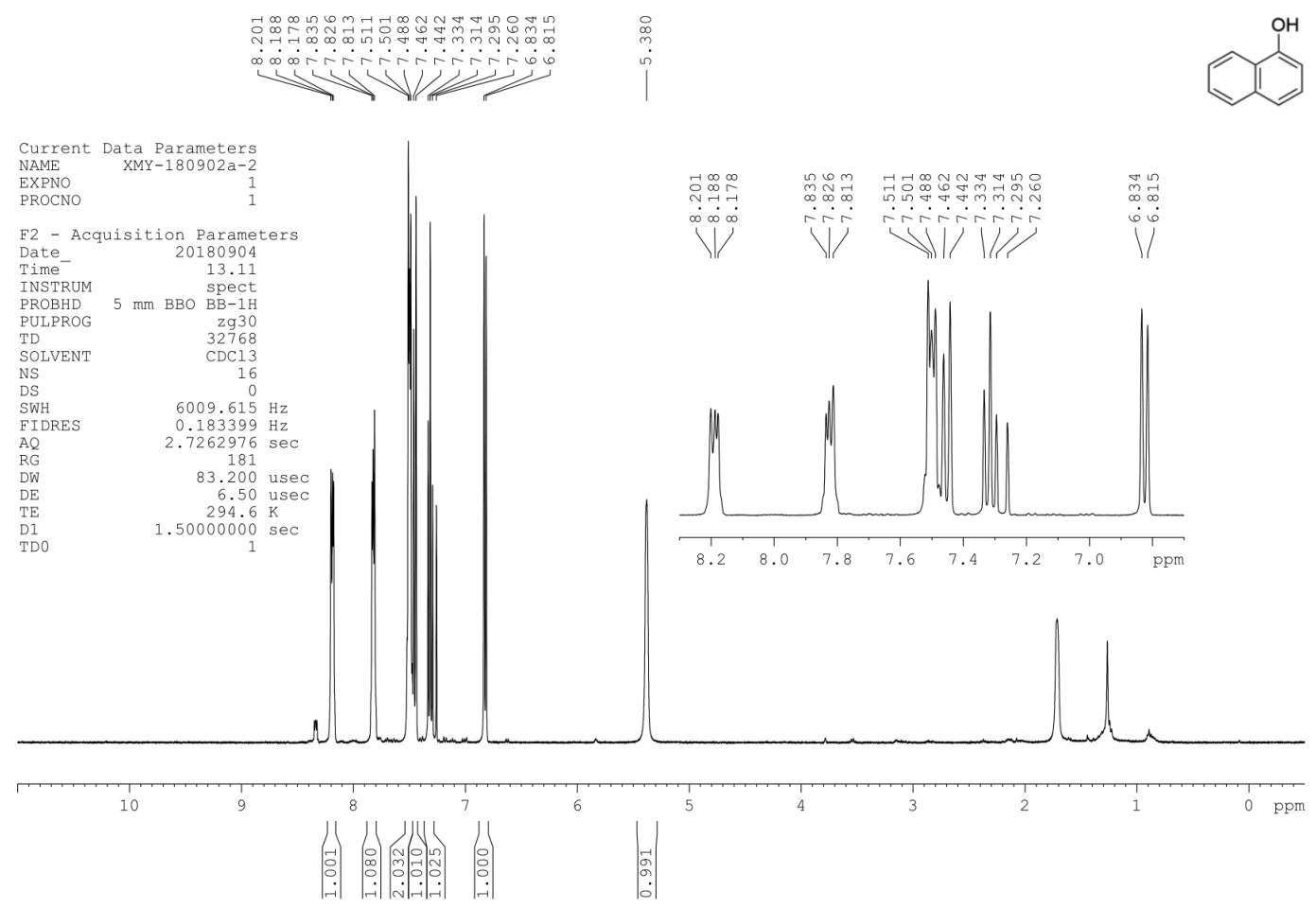


${ }^{13} \mathrm{C} \mathrm{NMR}$ of $6 \mathrm{w}, \mathrm{CDCl}_{3}, 100 \mathrm{MHz}, 22{ }^{\circ} \mathrm{C}$
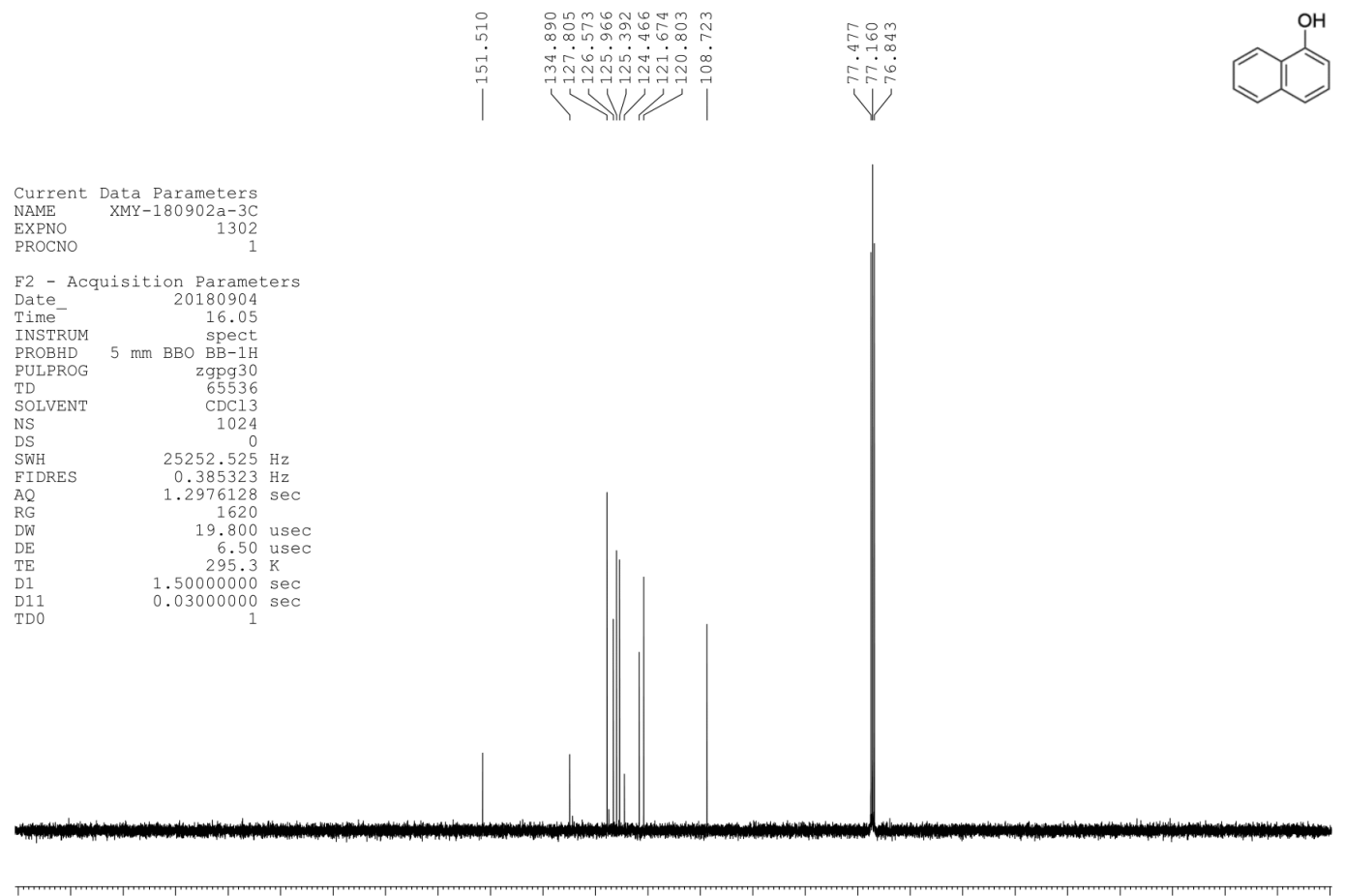

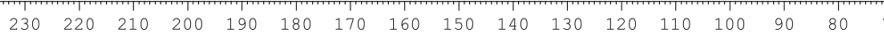

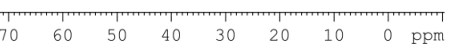

${ }^{1} \mathrm{H}$ NMR of $6 \mathrm{x}, \mathrm{CDCl}_{3}, 400 \mathrm{MHz}, 21^{\circ} \mathrm{C}$

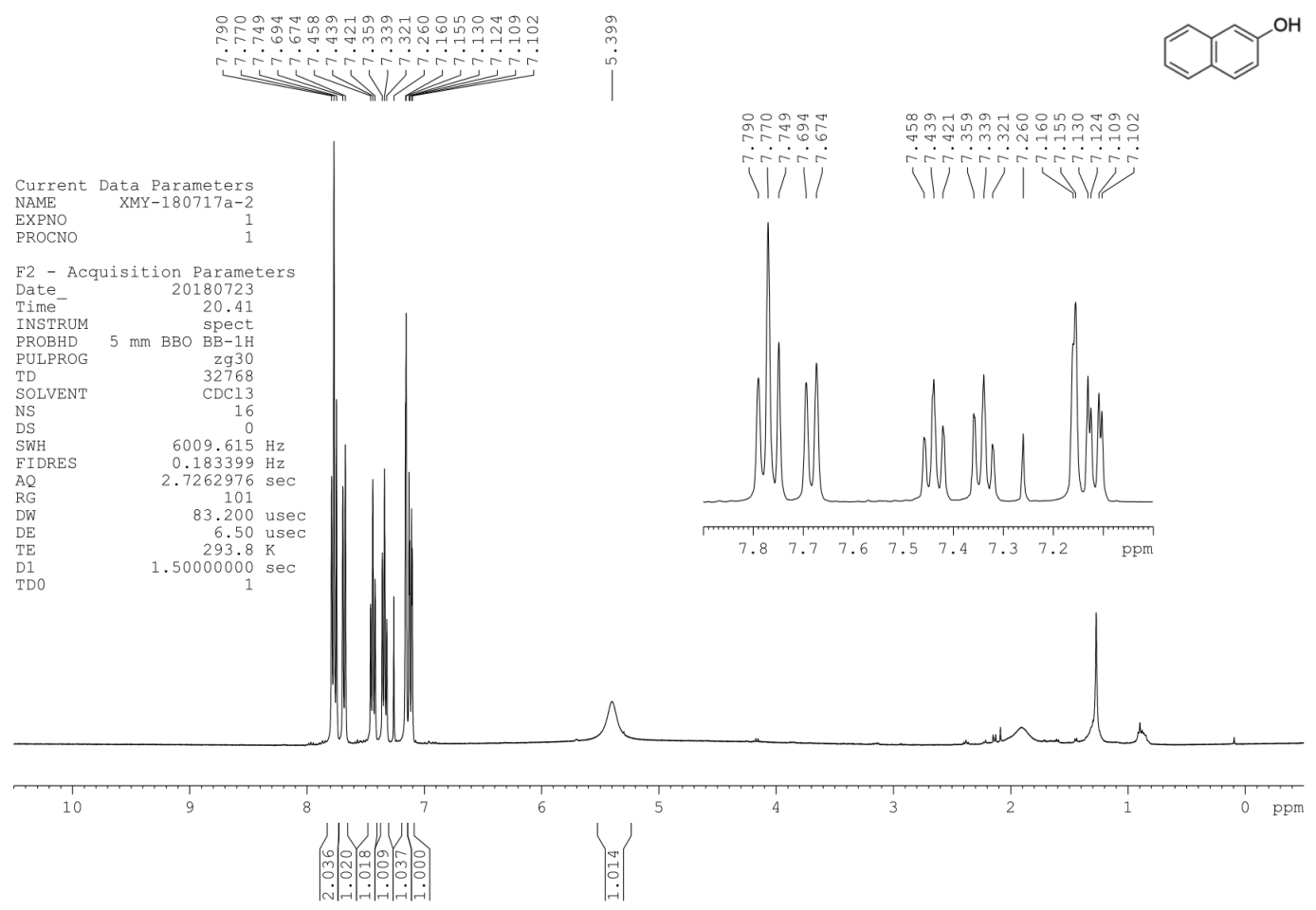


${ }^{13} \mathrm{C}$ NMR of $6 \mathrm{x}, \mathrm{CDCl}_{3}, 100 \mathrm{MHz}, 21^{\circ} \mathrm{C}$

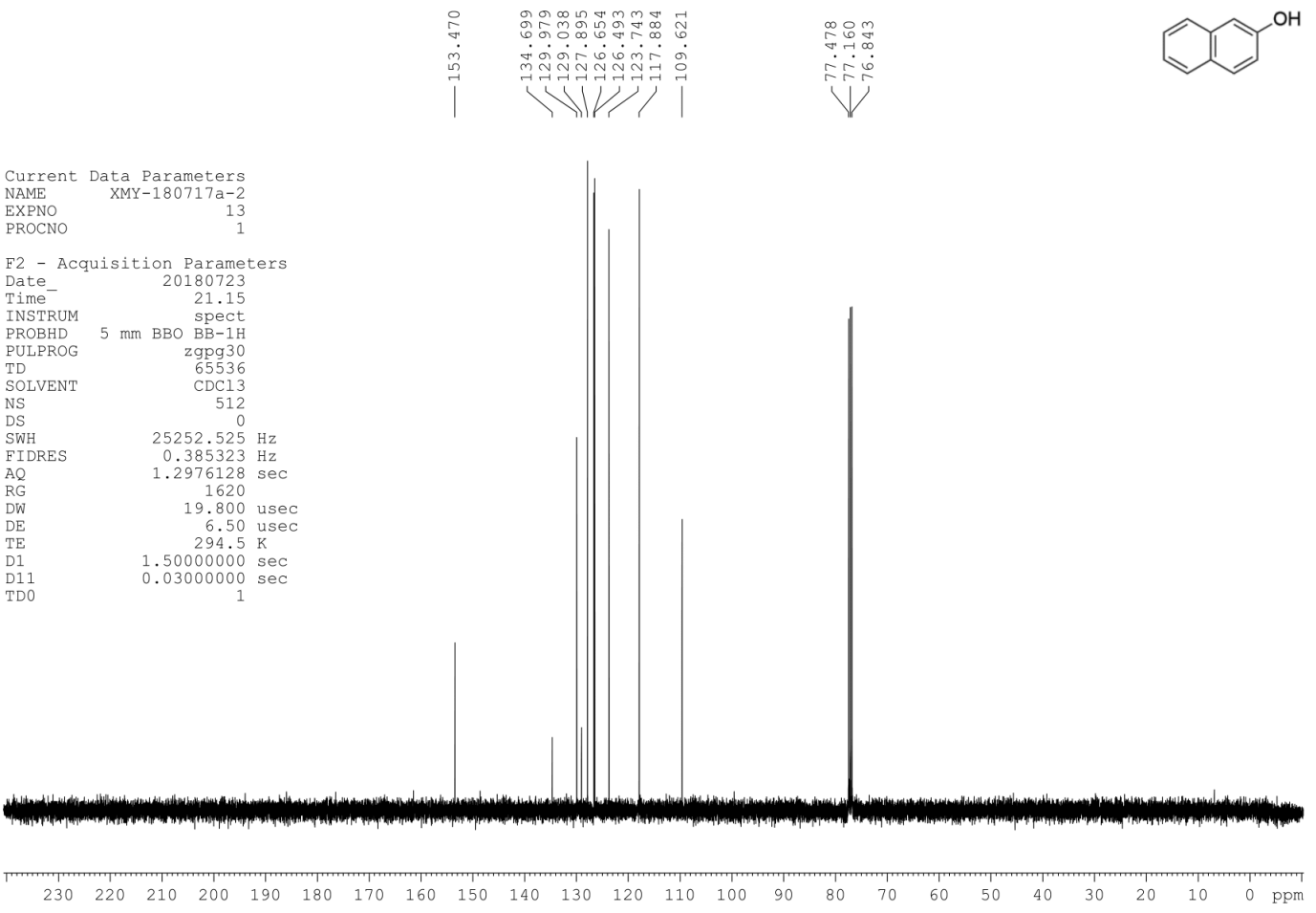

${ }^{1} \mathrm{H}$ NMR of $6 y, \mathrm{CDCl}_{3}, 400 \mathrm{MHz}, 21^{\circ} \mathrm{C}$
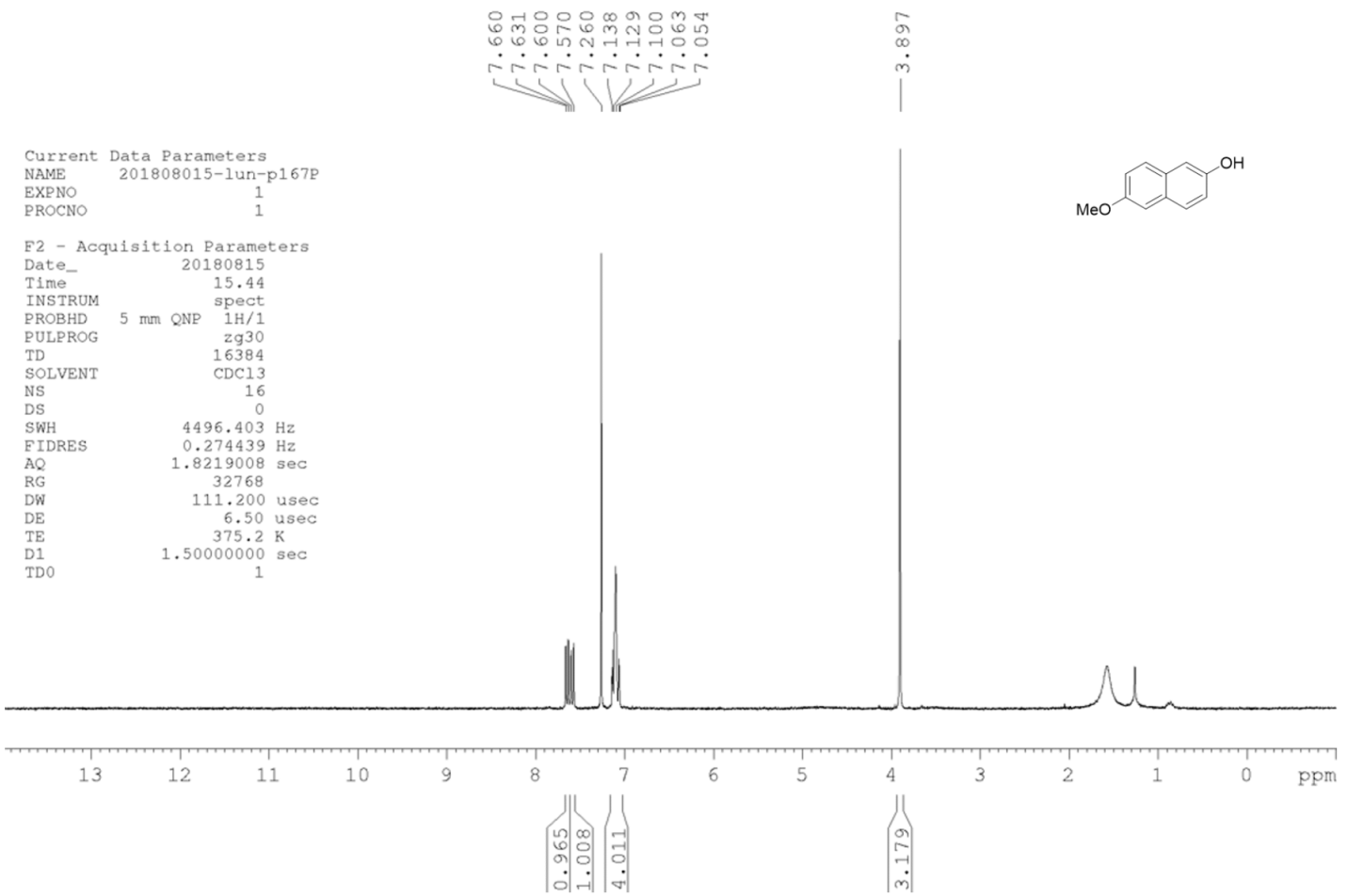
${ }^{13} \mathrm{C}$ NMR of $6 y, \mathrm{CDCl}_{3}, 100 \mathrm{MHz}, 25{ }^{\circ} \mathrm{C}$

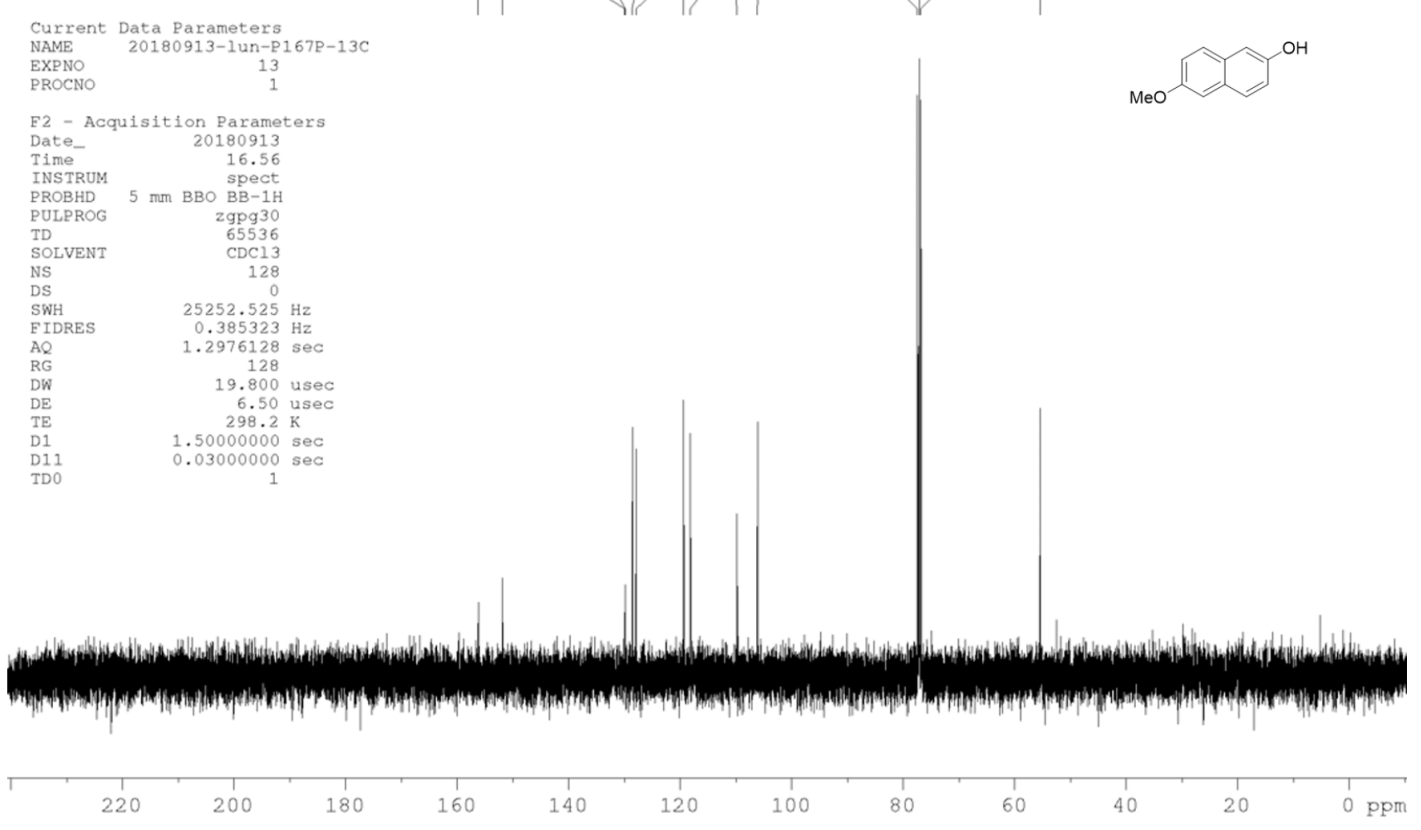

${ }^{1} \mathrm{H}$ NMR of $6 z, \mathrm{CDCl}_{3}, 400 \mathrm{MHz}, 21^{\circ} \mathrm{C}$

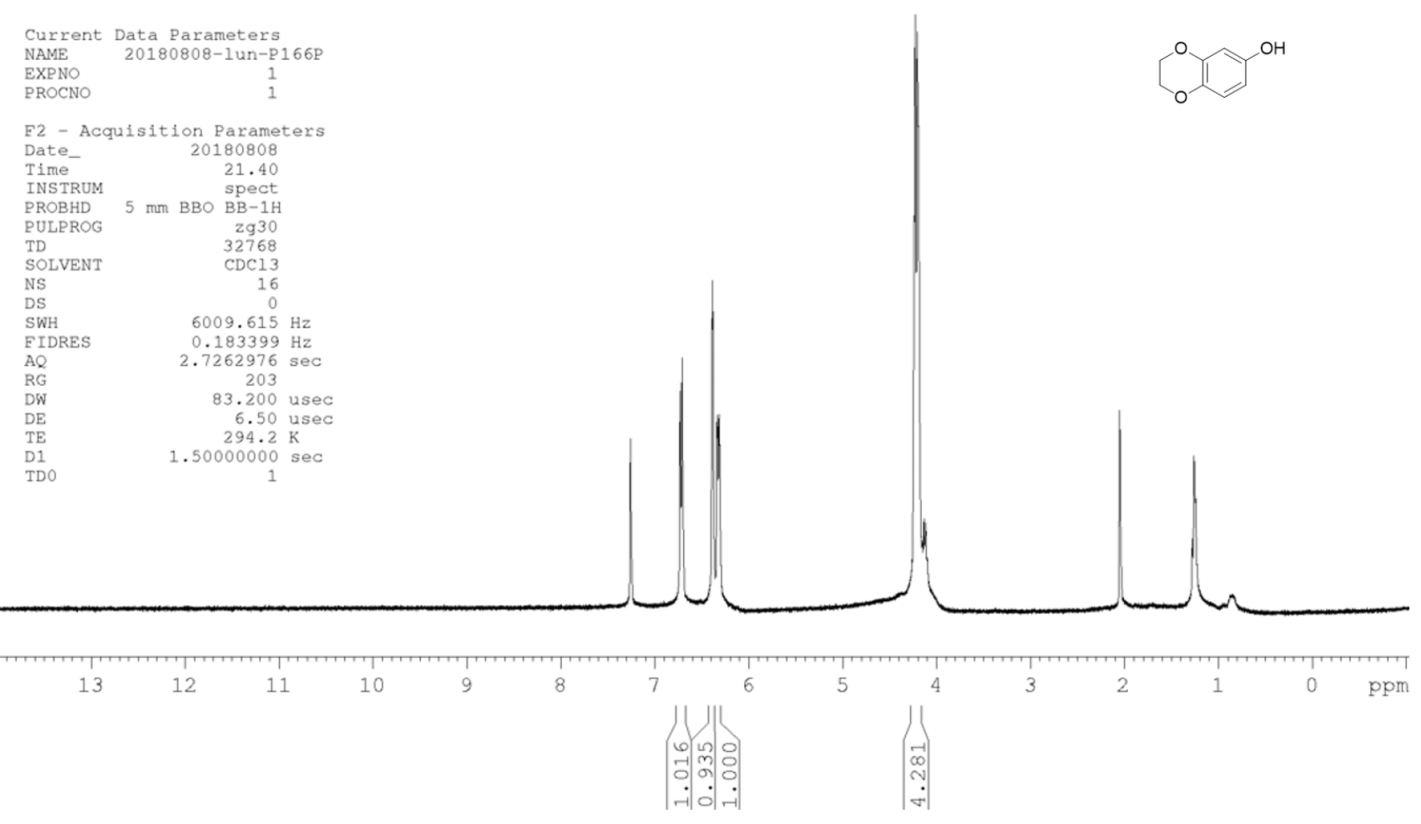


${ }^{13} \mathrm{C}$ NMR of $6 z, \mathrm{CDCl}_{3}, 100 \mathrm{MHz}, 25{ }^{\circ} \mathrm{C}$

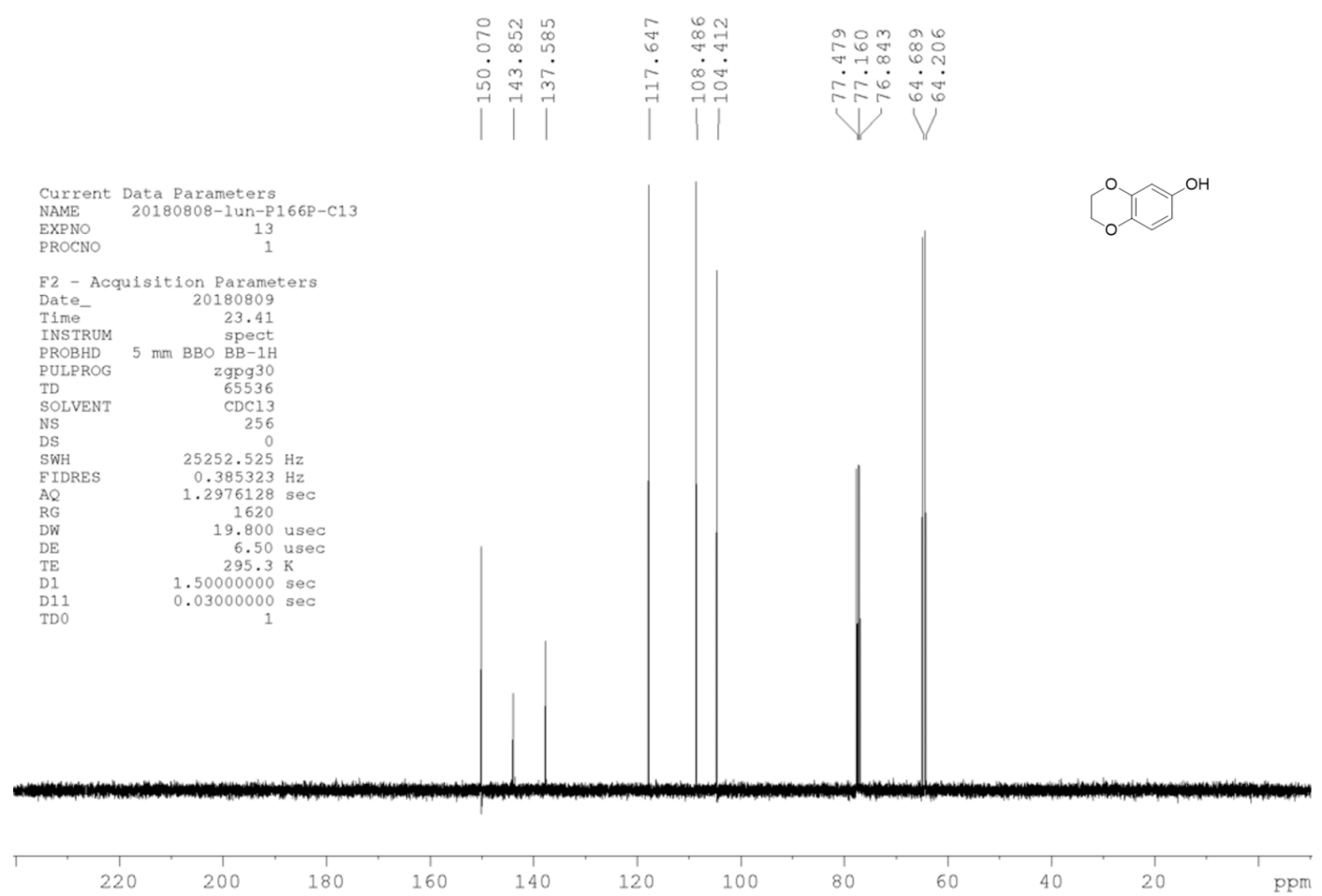

${ }^{1} \mathrm{H}$ NMR of $6 \mathrm{aa}, \mathrm{CDCl}_{3}, 400 \mathrm{MHz}, 21^{\circ} \mathrm{C}$

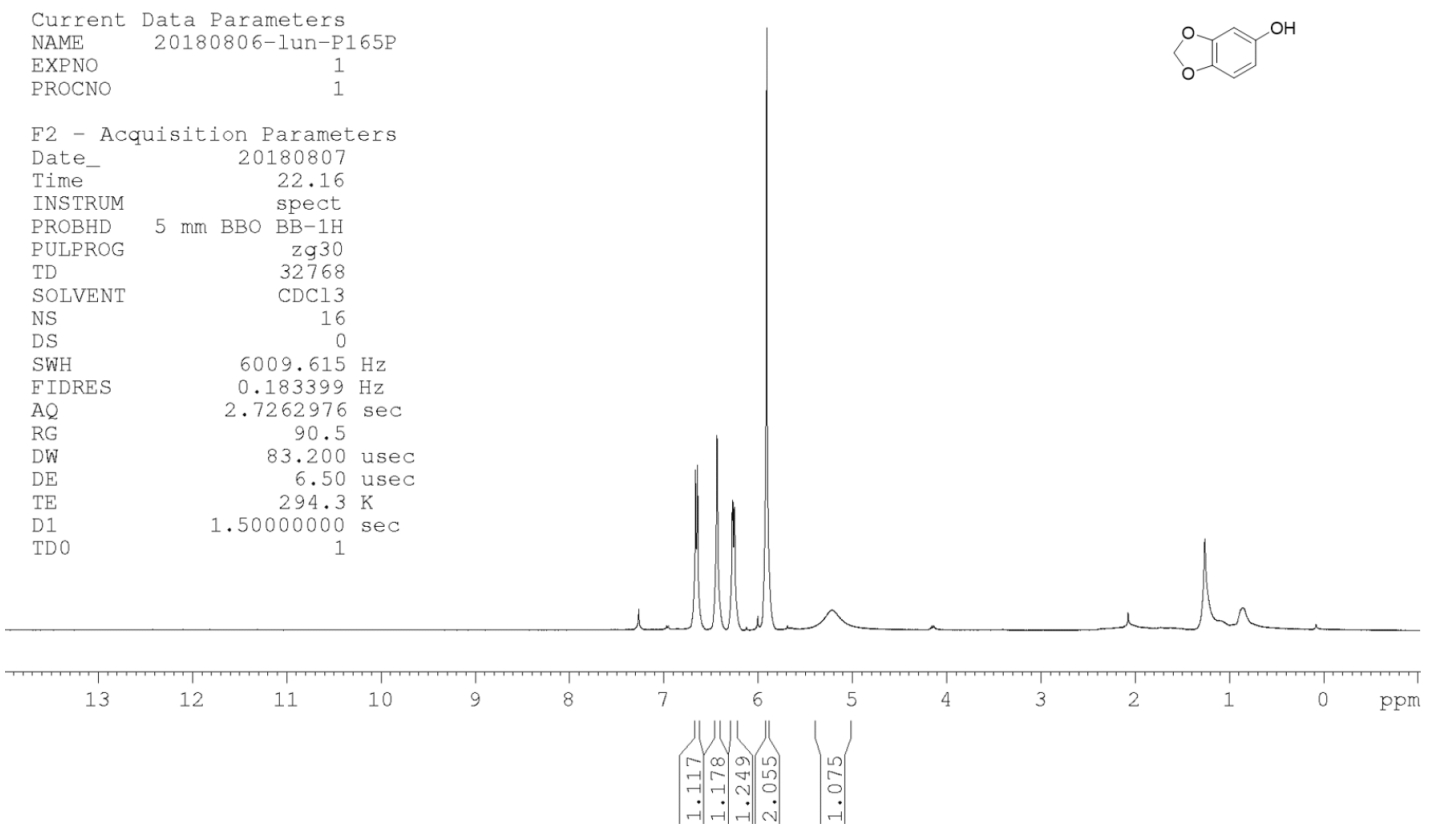


${ }^{13} \mathrm{C}$ NMR of $6 \mathrm{aa}, \mathrm{CDCl}_{3}, 100 \mathrm{MHz}, 25{ }^{\circ} \mathrm{C}$

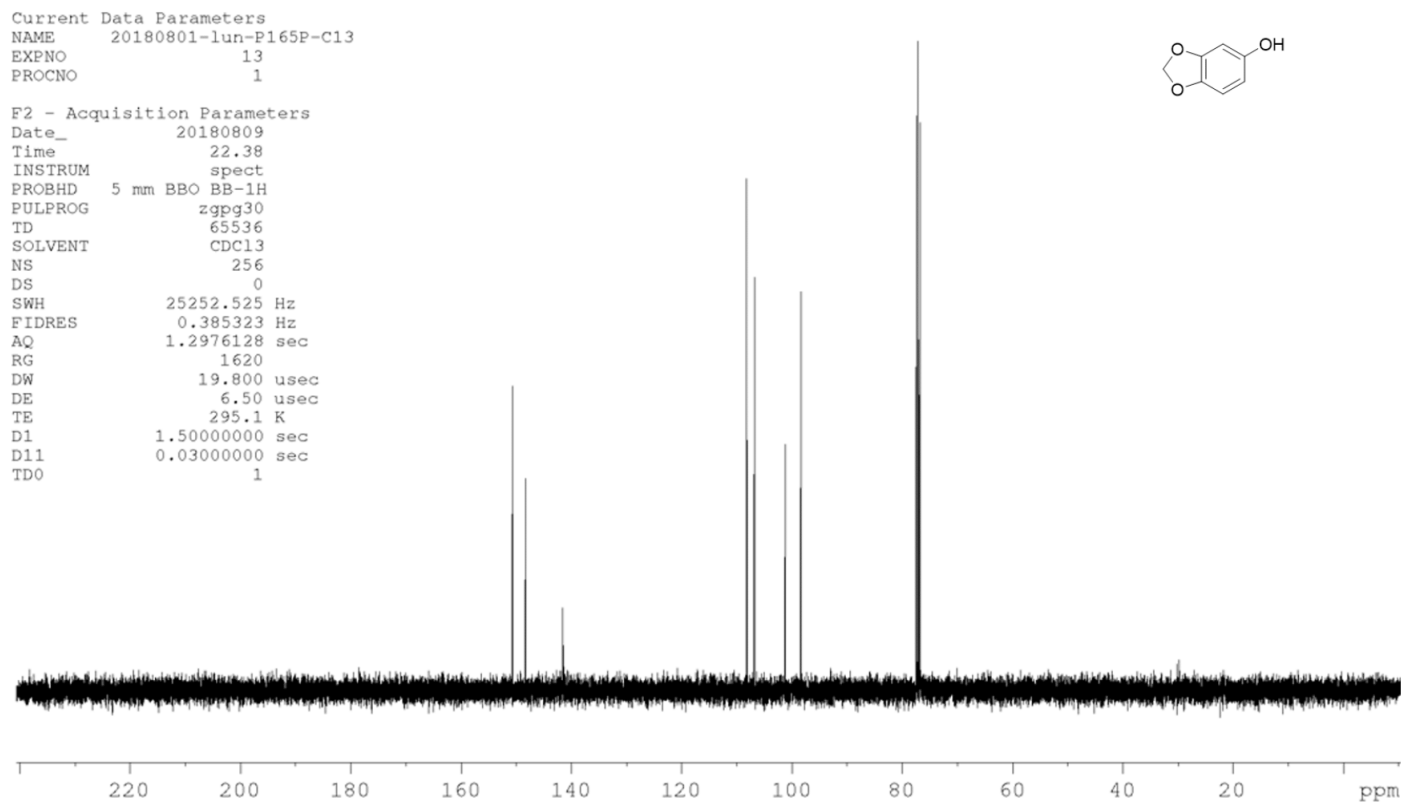

${ }^{1} \mathrm{H}$ NMR of $6 \mathrm{ab}, \mathrm{CDCl}_{3}, 400 \mathrm{MHz}, 2{ }^{\circ} \mathrm{C}$
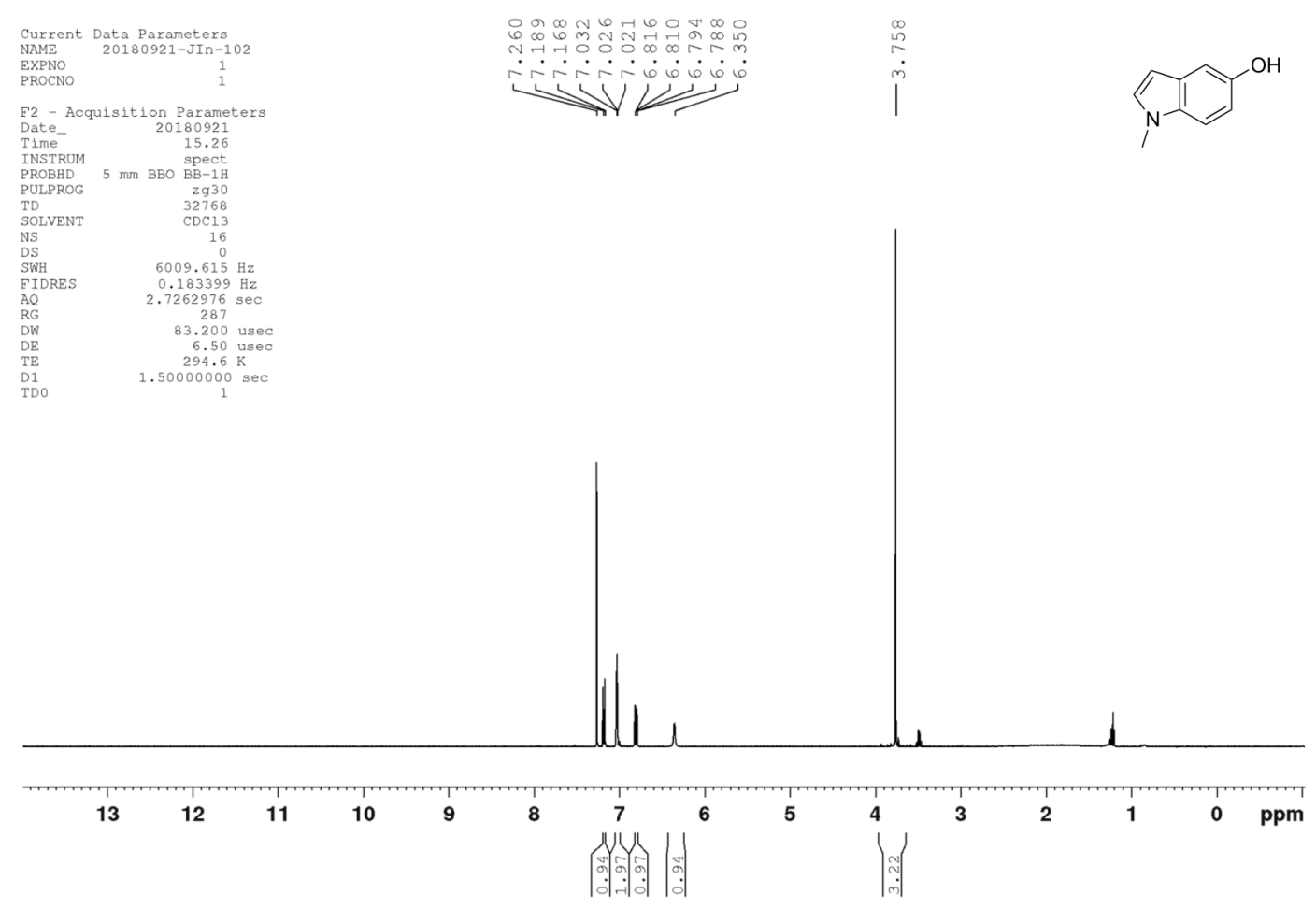
${ }^{13} \mathrm{C}$ NMR of $6 \mathrm{ab}, \mathrm{CDCl}_{3}, 100 \mathrm{MHz}, 25^{\circ} \mathrm{C}$
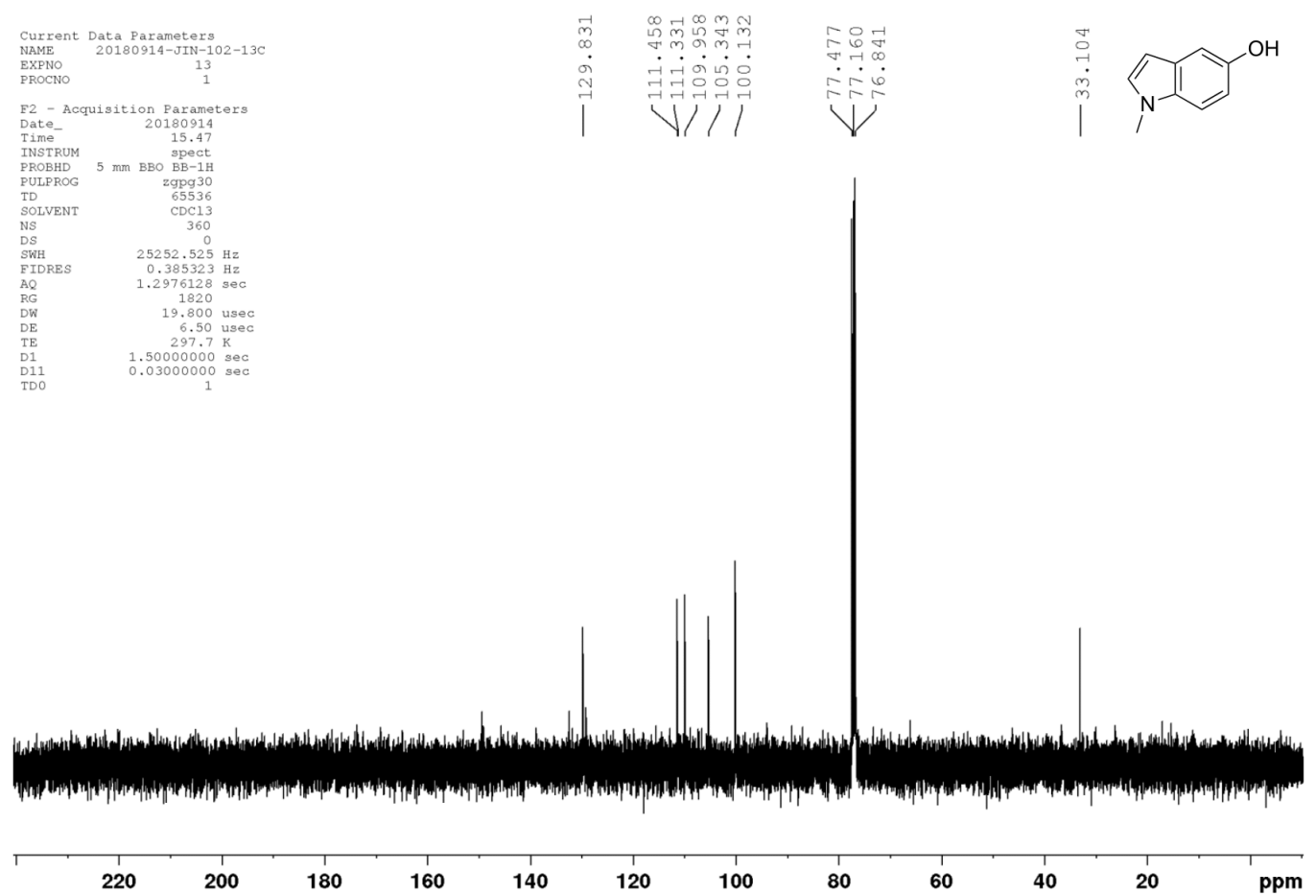

${ }^{1} \mathrm{H}$ NMR of 6ac, DMSO-d $6,400 \mathrm{MHz}, 21^{\circ} \mathrm{C}$

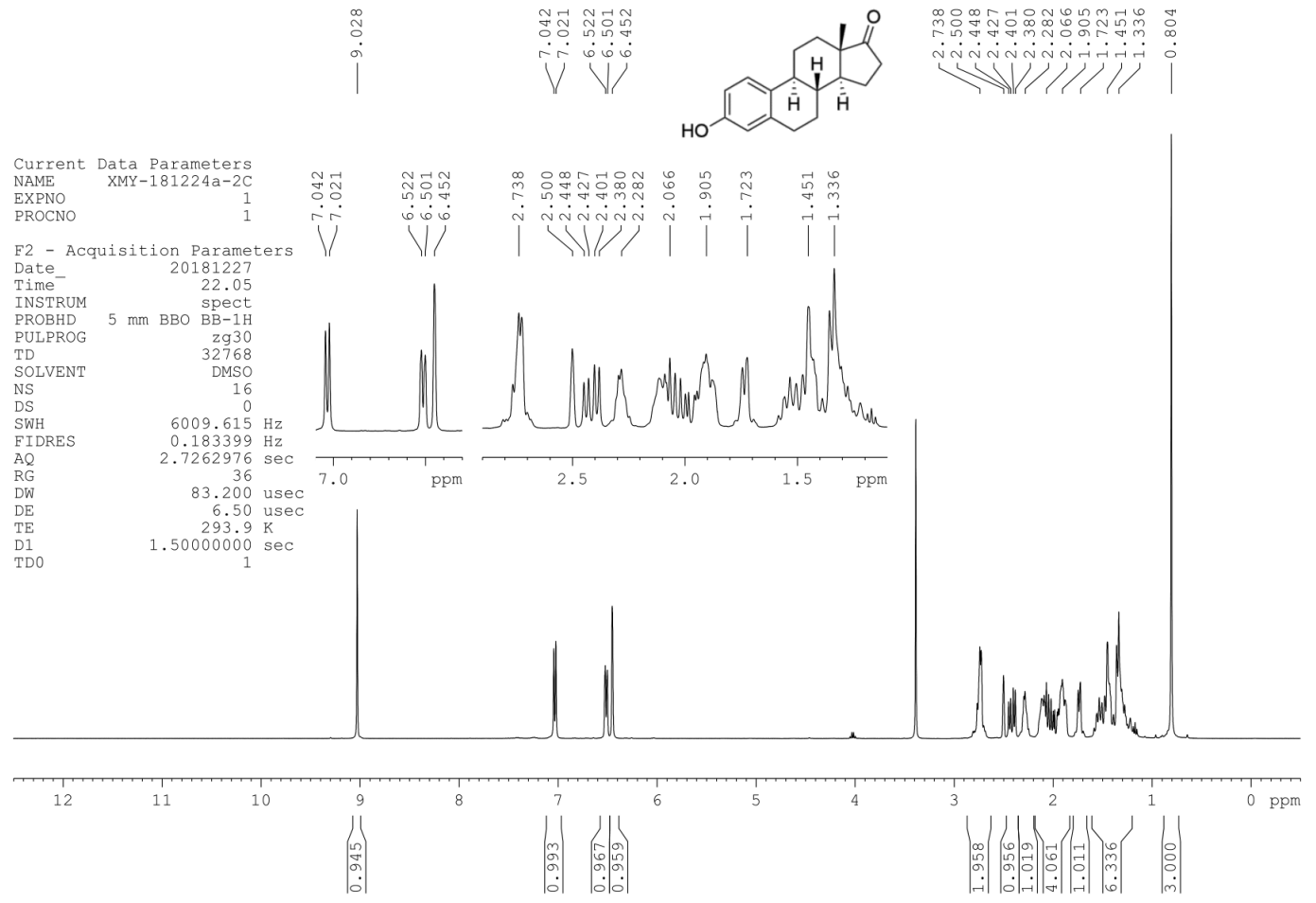


DEPT 135, DEPT 90 and ${ }^{13} \mathrm{C}$ NMR of 6ac, DMSO-d $6,100 \mathrm{MHz}, 2{ }^{\circ} \mathrm{C}$
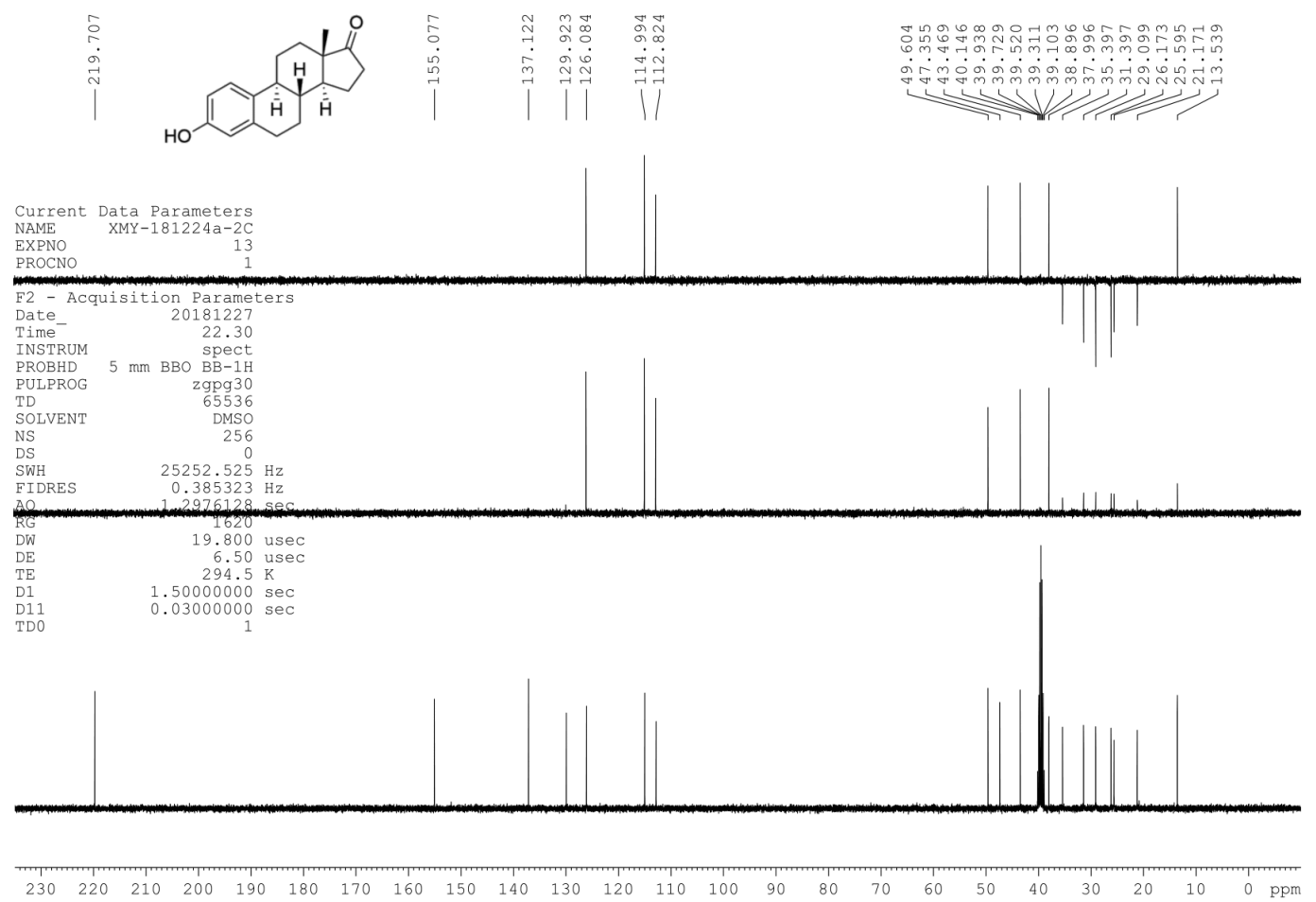

${ }^{1} \mathrm{H}$ NMR of $9, \mathrm{CDCl}_{3}, 400 \mathrm{MHz}, 20^{\circ} \mathrm{C}$

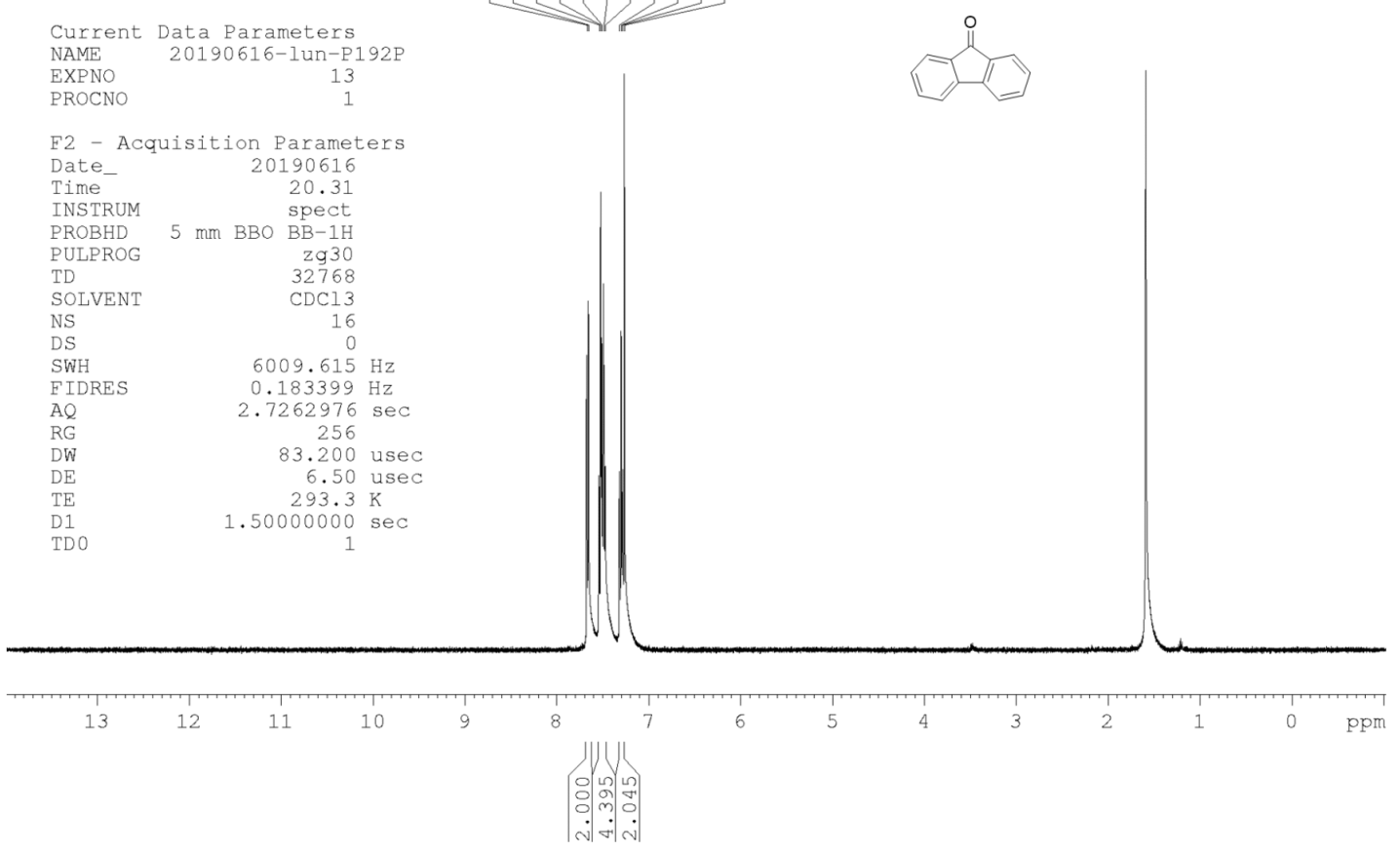


${ }^{13} \mathrm{C}$ NMR of $9, \mathrm{CDCl}_{3}, 100 \mathrm{MHz}, 20^{\circ} \mathrm{C}$

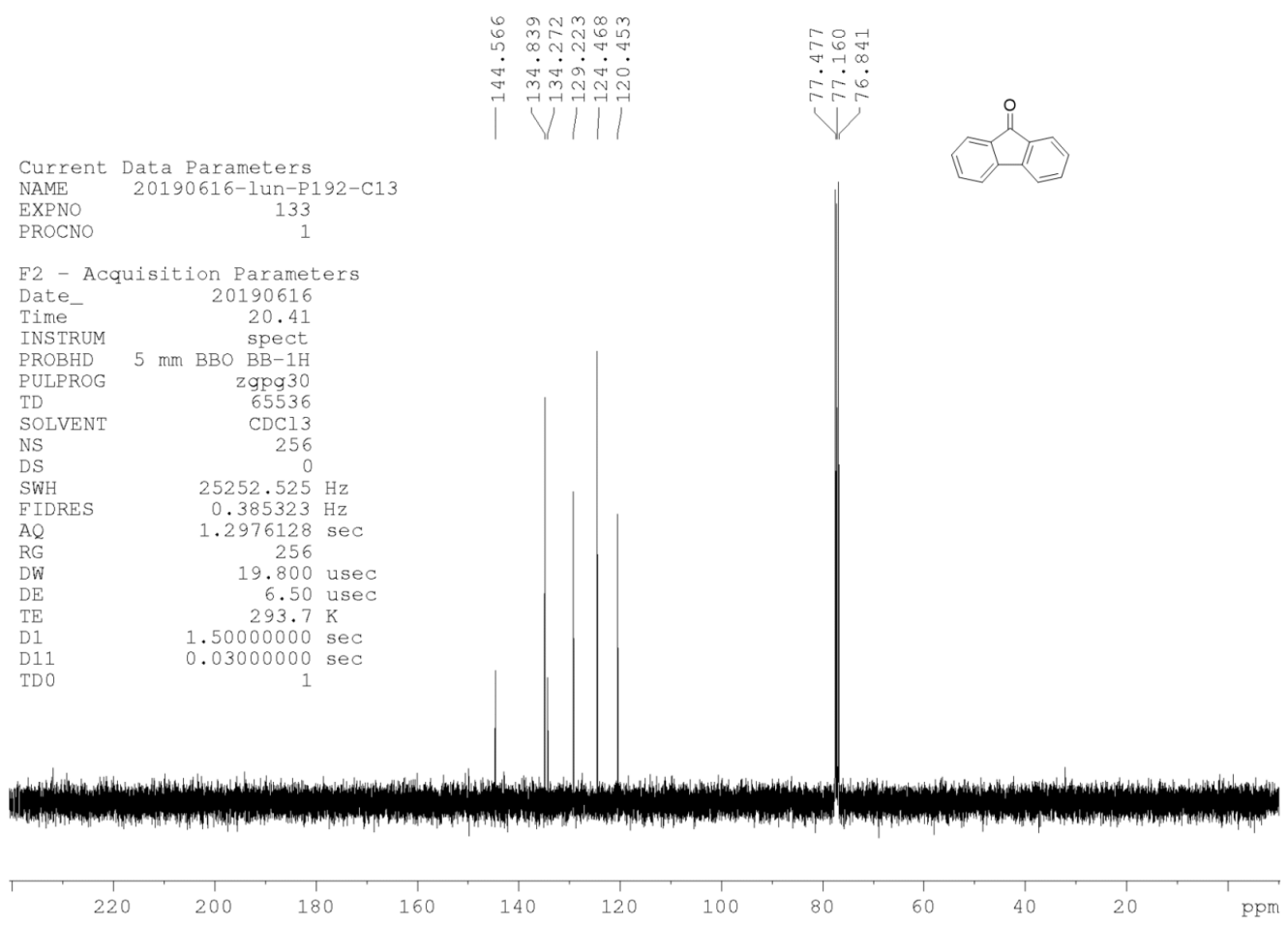

${ }^{1} \mathrm{H}$ NMR of $11, \mathrm{CDCl}_{3}, 400 \mathrm{MHz}, 21^{\circ} \mathrm{C}$
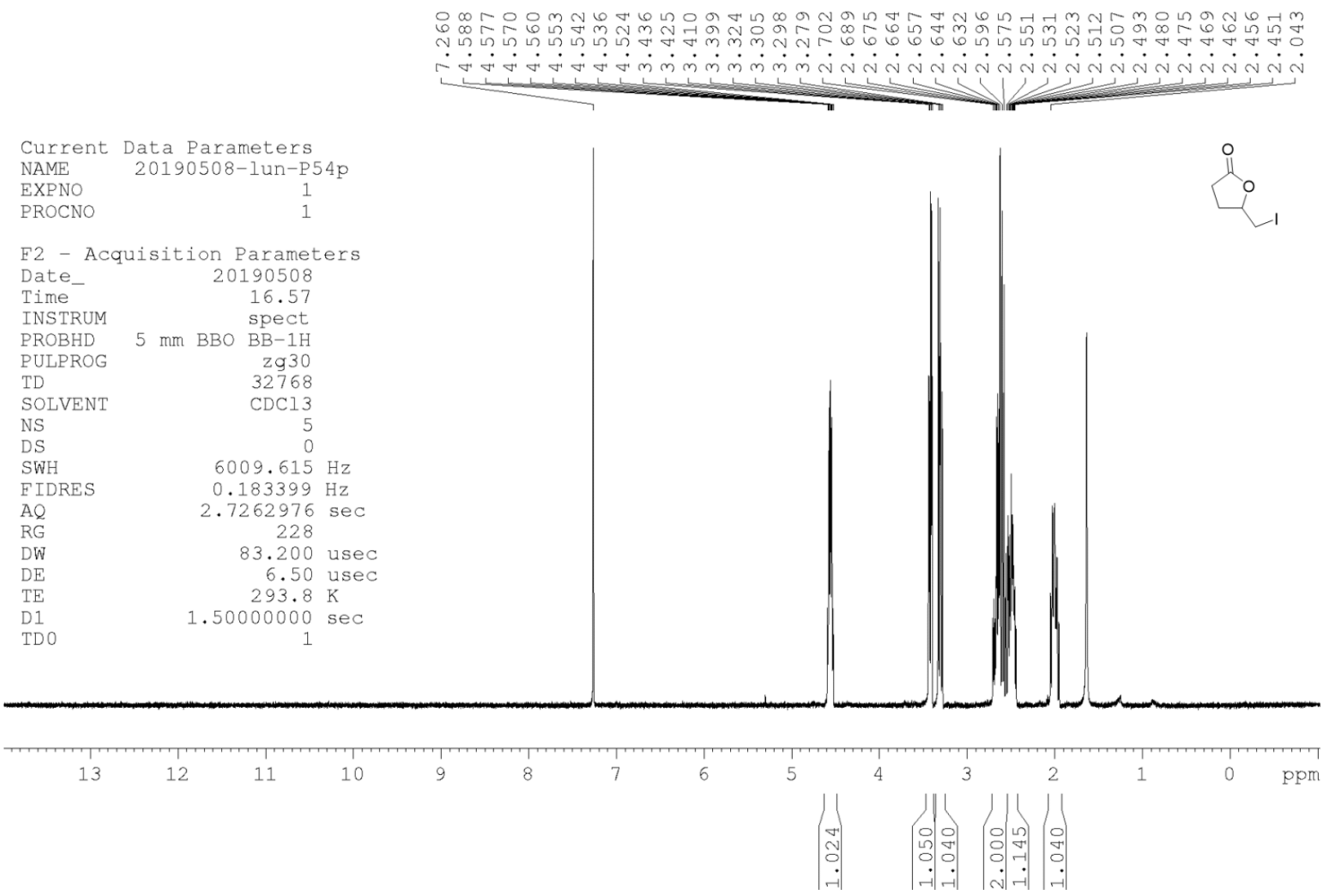
${ }^{13} \mathrm{C}$ NMR of $11, \mathrm{CDCl}_{3}, 100 \mathrm{MHz}, 21^{\circ} \mathrm{C}$

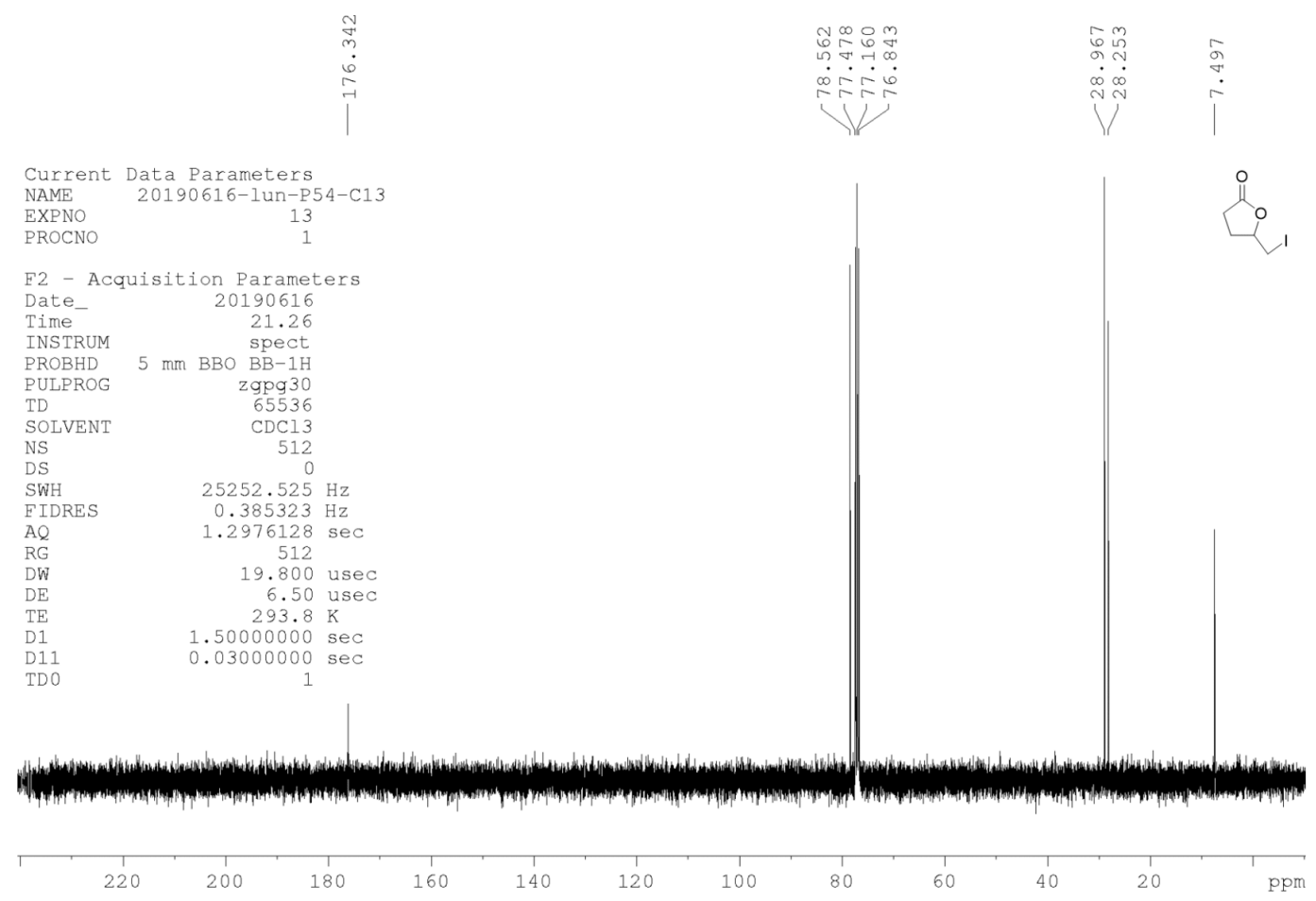

${ }^{1} \mathrm{H}$ NMR of $13, \mathrm{CDCl}_{3}, 400 \mathrm{MHz}, 21^{\circ} \mathrm{C}$
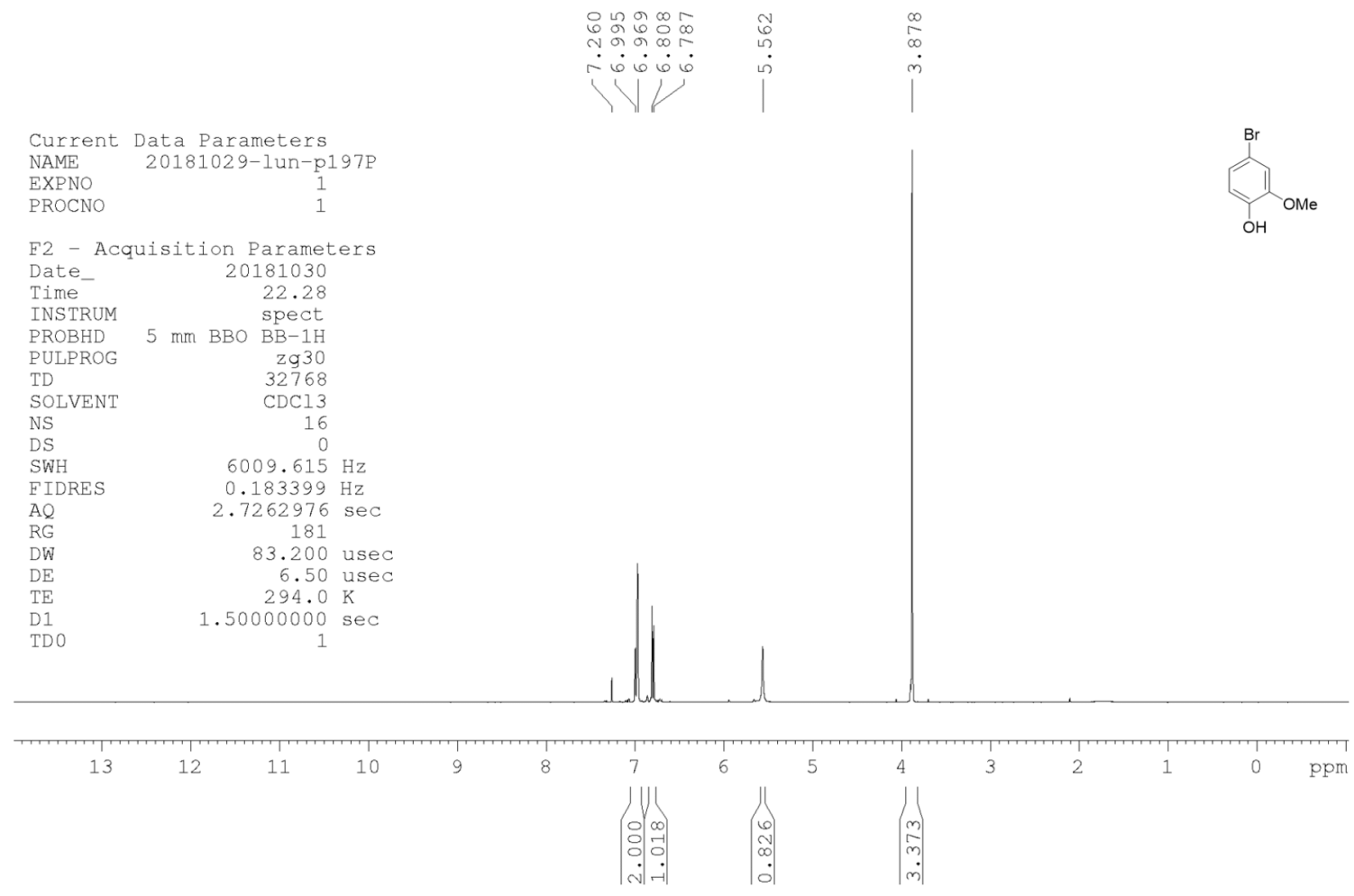
${ }^{13} \mathrm{C}$ NMR of $13, \mathrm{CDCl}_{3}, 100 \mathrm{MHz}, 21^{\circ} \mathrm{C}$
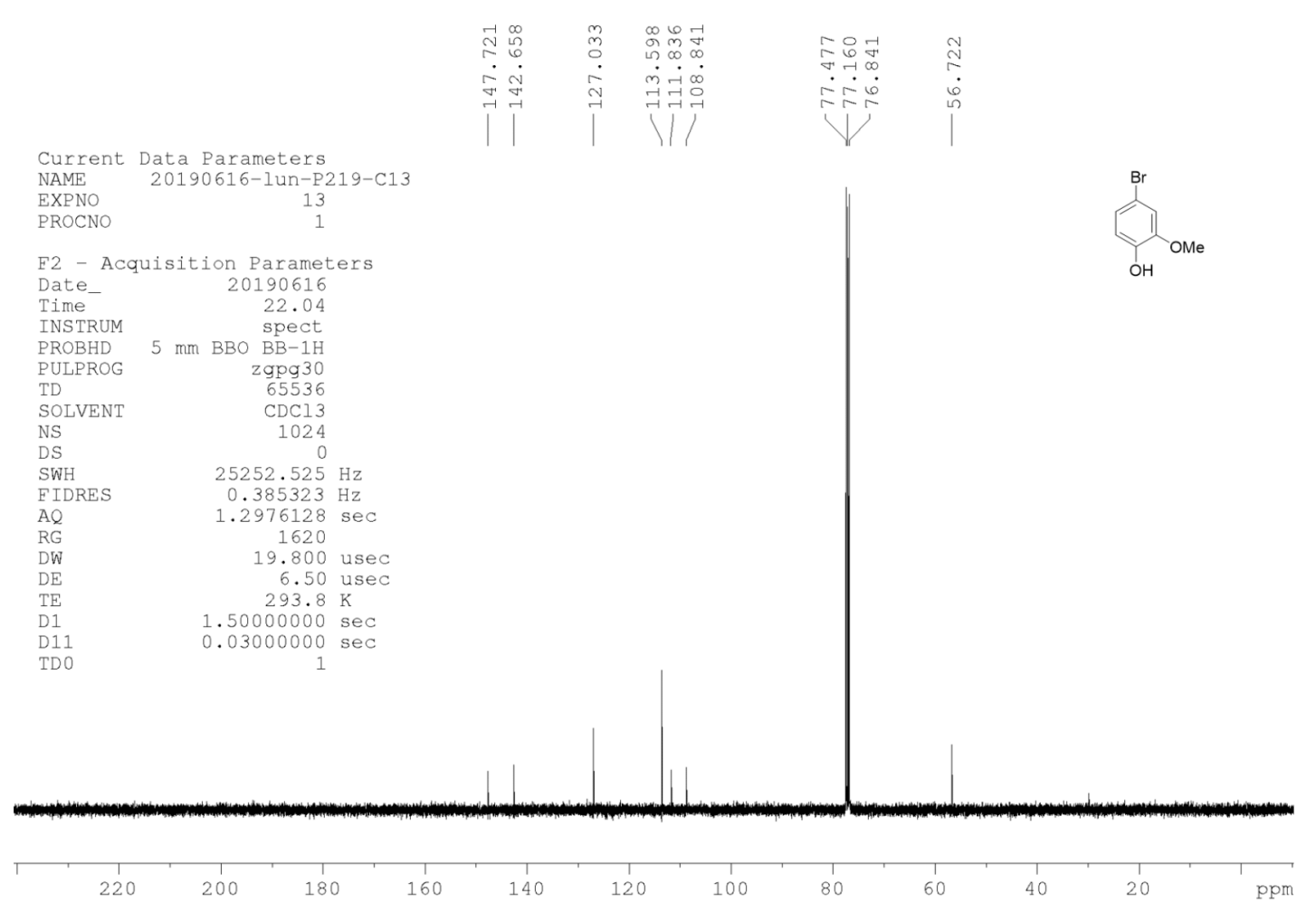